\title{
Mechanocontrol of cardiac growth and remodeling : mathematical and experimental analysis of normal and situs inversus totalis hearts
}

Citation for published version (APA):

Kroon, J. W. (2009). Mechanocontrol of cardiac growth and remodeling : mathematical and experimental analysis of normal and situs inversus totalis hearts. [Doctoral Thesis, Maastricht University]. Datawyse / Universitaire Pers Maastricht. https://doi.org/10.26481/dis.20090514jk

Document status and date:

Published: 01/01/2009

DOI:

10.26481/dis.20090514jk

Document Version:

Publisher's PDF, also known as Version of record

Please check the document version of this publication:

- A submitted manuscript is the version of the article upon submission and before peer-review. There can be important differences between the submitted version and the official published version of record.

People interested in the research are advised to contact the author for the final version of the publication, or visit the DOI to the publisher's website.

- The final author version and the galley proof are versions of the publication after peer review.

- The final published version features the final layout of the paper including the volume, issue and page numbers.

Link to publication

\footnotetext{
General rights rights.

- You may freely distribute the URL identifying the publication in the public portal. please follow below link for the End User Agreement:

www.umlib.nl/taverne-license

Take down policy

If you believe that this document breaches copyright please contact us at:

repository@maastrichtuniversity.nl

providing details and we will investigate your claim.
}

Copyright and moral rights for the publications made accessible in the public portal are retained by the authors and/or other copyright owners and it is a condition of accessing publications that users recognise and abide by the legal requirements associated with these

- Users may download and print one copy of any publication from the public portal for the purpose of private study or research.

- You may not further distribute the material or use it for any profit-making activity or commercial gain

If the publication is distributed under the terms of Article 25fa of the Dutch Copyright Act, indicated by the "Taverne" license above, 


\section{Mechanocontrol of cardiac growth and remodeling}

Mathematical and experimental analysis of normal and situs inversus totalis hearts 
() Wilco Kroon, Maastricht 2009

ISBN: 9780952788241

Universitaire Pers Maastricht 


\title{
Mechanocontrol of cardiac growth and remodeling
}

\author{
Mathematical and experimental analysis of \\ normal and situs inversus totalis hearts
}

\author{
PROEFSCHRIFT \\ ter verkrijging van de graad van doctor \\ aan de Universiteit Maastricht, \\ op gezag van de Rector Magnificus, \\ Prof. mr. G.P.M.F. Mols \\ volgens het besluit van het College van Decanen \\ in het openbaar te verdedigen \\ op donderdag 14 mei 2009 om 14:00 uur \\ door \\ Jacob Willem Kroon \\ Geboren op 7 juni 1981 \\ te Velp
}

$\underbrace{\underbrace{\top}}_{\substack{\text { UNIVERSITARE } \\ \text { PER MASTRICHT }}}$ 


\section{Promotor}

Prof. dr. ir. T. Arts

\section{Copromotores}

Dr. T. Delhaas

Dr. ir. P.H.M. Bovendeerd, Technische Universiteit Eindhoven

\section{Beoordelingscommissie}

Prof. dr. ir. F.N. van de Vosse (voorzitter)

Prof. dr. H.J.G.M. Crijns

Prof. dr. A.C. Gittenberger-de Groot, Leids Universitair Medisch Centrum

Prof. dr. J.H. Omens, University of California San Diego

Prof. dr. G.J. van der Vusse

Financial support for the publication of this thesis by the Netherlands Heart Foundation and the "Stichting Hartsvrienden RESCAR" is gratefully acknowledged. 


\section{Table of contents}

\section{Chapter 1}

Background and aim of the thesis

\section{Chapter 2}

Structure and torsion of the normal and SIT cardiac left ventricle:

I. Experimental data in humans

\section{Chapter 3}

Structure and torsion of the normal and SIT cardiac left ventricle:

II. Modeling cardiac adaptation to mechanical load

Chapter 4

Computational analysis of the myocardial structure:

Adaptation of myofiber orientations through deformation in three dimensions.

\section{Chapter 5}

Adaptation of myofiber orientations through deformation:

Comparison between shear strains in model and experiment.

\section{Chapter 6}

Computational modeling of volumetric soft tissue growth:

Application to the cardiac left ventricle

\section{Chapter 7}

General discussion

Summary

Samenvatting

Dankwoord

Curriculum Vitae 



\section{Background and aim of the thesis}




\subsection{GENERAL INTRODUCTION}

The heart is a cavernous muscular organ that is responsible for circulating blood through the body to exchange oxygen, nutrients, and metabolites. Pump capacity of the heart is determined by its size, composition, and structure. Normally, growth and remodeling (G\&R) of cardiac tissue during embryonic development, as well as during normal adult physiology, results in a heart that is well able to deliver the pump capacity needed for adequate blood circulation through the body. Due to disease, however, pump capacity may diminish. Initially, G\&R will adapt the tissue to restore pump capacity. However, as disease progresses, G\&R may no longer be able to compensate and pump capacity becomes insufficient to comply with the needs of the body.

Mechanical tissue load plays an important regulatory role in cardiac G\&R (25, $32,46,67,68,73)$. For instance, gene expression in the embryonic heart is related to mechanical shear exerted on the tissue by the circulating blood (32). Furthermore, an exercise-related chronic increase in mechanical load is found to result in an increase of cavity and wall volume (athlete's heart) (25). Despite these and other in vitro and in vivo observations, the mechanical stimuli and their related responses are not completely elucidated yet, mainly due to limitations in the experimental methodology. Additional knowledge is needed to improve our understanding of cardiac development as well as to predict the long-term outcome of disease and the effect of possible treatment strategies. In this thesis, we develop and apply mathematical models to gain more insight into the mechanisms involved in mechanically induced cardiac G\&R. Myofiber reorientation is the primary process that has been studied. Volumetric growth is considered as a secondary G\&R process.

In this chapter we provide a brief description of cardiac anatomy and structure. Next, we describe the major determinants of mechanical tissue load and address their relation to cardiac G\&R. Subsequently, we elaborate on the use of mathematical models to investigate stimulus-response relations. This chapter concludes with the specific aims and outline of the thesis.

\subsection{BASIC CARDIAC ANATOMY AND PHYSIOLOGY}

\subsubsection{Anatomy and structure}

The heart contains four hollow chambers with muscular walls (Fig 1.1A). Blood returning from the body is first collected in the right atrium (RA). Next, it passes the tricuspid valve entering the right ventricle (RV). The RV then propels blood through the pulmonary valve into the pulmonary circulation where it is oxygenized in the lungs. Oxygen-saturated blood returning from the lungs is collected in the 
left atrium (LA), passes the mitral valve, and arrives in the left ventricle (LV). Then, a powerful LV contraction pumps the blood through the aortic valve into the aorta after which it is distributed to the body organs via the systemic circulation.

Geometrically, the LV may be considered as a thickwalled truncated ellipsoid, i.e., the long axis contours of the LV are elliptical, while the short axis contours are virtually circular. The relatively thinwalled $R V$ is connected to the outer layers of the LV and covers nearly half the LV outer surface. The cavities of both ventricles are separated by the interventricular septum. The ventricles are separated from the atria by the basal plane.

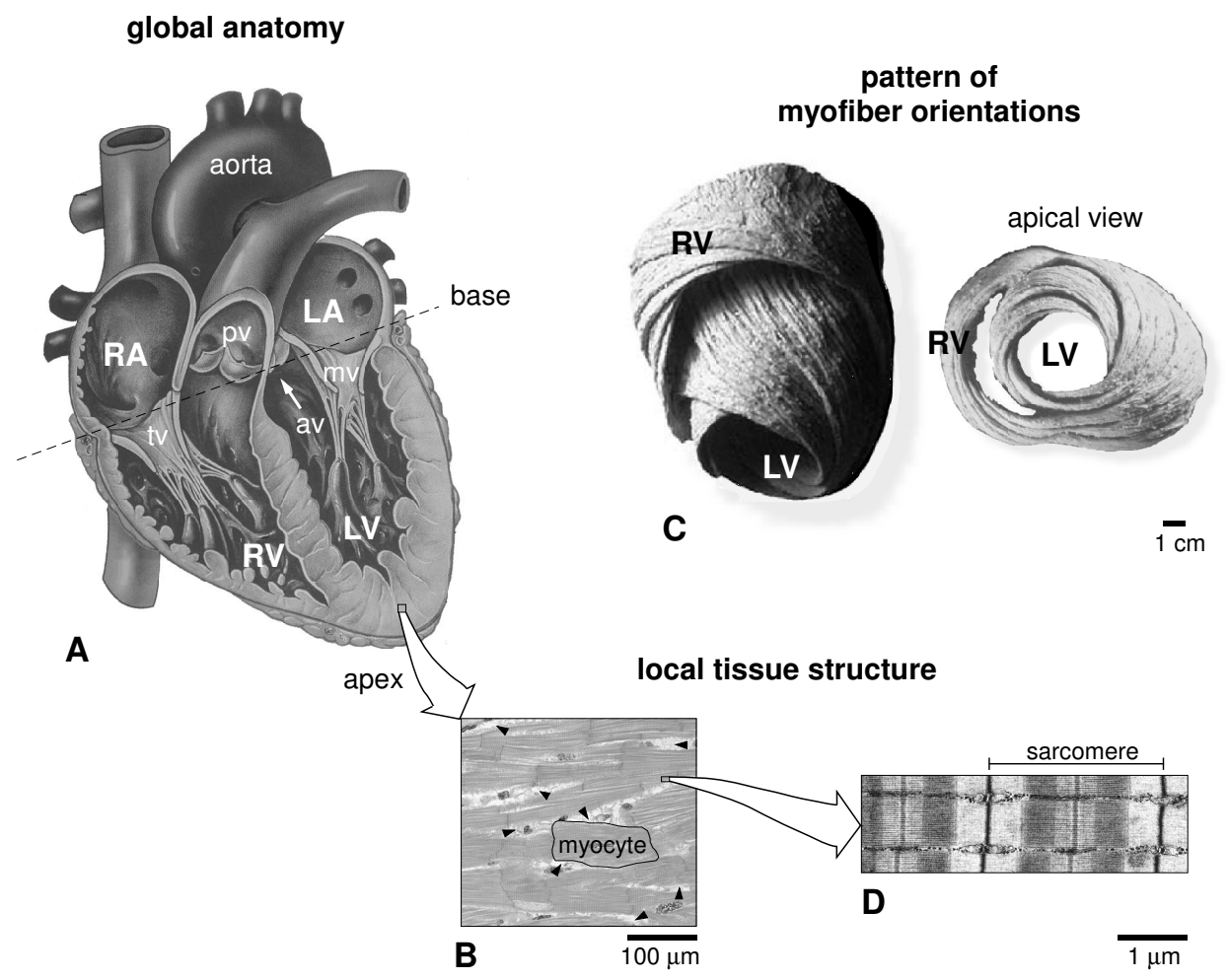

Figure 1.1. Anatomy and structure of the heart. A Cross-section of the heart along its long axis. $L A=$ left atrium; $L V=$ left ventricle; $R A=$ right atrium; $R V=$ right ventricle; av=aortic valve; $\mathrm{mv}=$ mitral valve; $\mathrm{pv}=$ pulmonary valve; $\mathrm{tv}=$ tricuspid valve. Adapted from (47). B Microscopy image of the cardiac tissue showing aligned myocytes. Arrowheads indicate the extracellular matrix. C Specimen showing the helical orientation of the myofibers as made visible by blunt dissection. Across the wall, the myofiber helix changes from a left-handed one in the outer layers to a right-handed one in the inner layers. Near the apex and base myofibers cross over between the inner and outer layers. Adapted from $(74,75)$. D Microscopy image of the force-generating sarcomeres inside the myocytes. 
Myocytes and fibroblasts constitute the majority of cells in the heart (40). Locally, myocytes are more or less aligned to form myofibers (Fig 1.1B). The orientation of these myofibers has a distinct pattern that appears similar across various animal species (Fig 1.1C). Within the LV, myofibers at the inner surface normally follow a right-handed helix around the cavity. Myofibers gradually change their orientation across the wall towards a left-handed helix at the outer surface. Near the apex and the base, the myofibers cross over between inner and outer layers, thus having a significant transmural component in their orientation $(26,31,65)$.

Inside the myocytes, contractile force is generated by sarcomeres (Fig 1.1D). The myocytes are surrounded and connected to a fibrous network mainly consisting of collagen, called the extra-cellular matrix (ECM). The ECM plays an important role in transmitting sarcomere-generated force to the ventricular chamber $(8,14,15)$. The ECM furthermore acts as a scaffold for the myofibers, reinforcing the mutual alignment of the myocytes. The ECM also connects myocytes to neighboring coronary vasculature. Fibroblasts play a vital role in the regulation of ECM composition and structure (63).

\subsubsection{Physiology and mechanical load}

A cardiac (pump) cycle consists of four phases; filling, isovolumic contraction, ejection and isovolumic relaxation. During filling, blood enters the LV through the mitral valve, resulting in a rise in cavity pressure. When the myofibers in the wall start to contract, LV cavity pressure increases further to a point where it exceeds the pressure inside the LA. Consequently, the mitral valve closes. Further contraction of the myofibers causes an additional increase in pressure. Since both the mitral valve and the aortic valve are closed now, cavity volume remains constant. The isovolumic contraction phase continues until LV cavity pressure exceeds aortic pressure. Then, the aortic valve opens and ejection starts. The amount of blood that is ejected into the aorta and the pressure at which it is ejected, are not only determined by total pump force generated by the LV, but also by hemodynamic load as imposed by the systemic circulation. Upon myofiber relaxation, cavity pressure drops to a point where the aortic valve closes and isovolumic relaxation begins. After further relaxation of the myofibers, LV cavity pressure drops below the pressure inside the LA. Consequently, the mitral valve reopens, the next cardiac cycle starts, and the LV is filled again.

During the cardiac cycle a loop is formed by the LV cavity pressure as a function of LV cavity volume (Fig 1.2A). This pressure-volume loop represents the global mechanical load of the LV during a cardiac cycle. Analogously, local mechanical load of the tissue during the cardiac cycle is represented by the myofiber stress as a function of myofiber strain (stress-strain loop, Fig 1.2B). Myofiber stress is defined as force along the myofiber axis per unit of cross-sectional area. Myofiber strain is defined as change in myofiber length relative to a certain reference. 
The pressure-volume loop and the stress-strain loop are bounded by two curves. These curves quantify the passive and active mechanical behavior of the heart. Myofiber stress in passive cardiac tissue increases about exponentially with myofiber strain $(19,39,60)$. At low sarcomere lengths, tissue stiffness is mainly determined by components of the myocyte cytoskeleton such as titin (30). At larger sarcomere lengths, collagen in the ECM becomes stretched, thereby increasing tissue stiffness and preventing overstretching of the myocardium during filling. Furthermore, passive mechanical behavior of cardiac tissue is anisotropic, i.e., along the myofiber axis, tissue is significantly stiffer than in directions perpendicular to the myofiber axis. In the actively contracting state, cardiac tissue shows a more linear relation between myofiber stress and strain. Local force actively generated by the myofibers depends, among other parameters, on time after activation, sarcomere length, sarcomere shortening velocity $(18,41)$, as well as on the boundary conditions imposed by the surrounding tissue.

The quantitative relation between stress and strain at the local tissue level and pressure and volume at the organ level is not straightforward. To clarify this relation researchers have used mathematical analyses. In such an analysis, the ratio of cavity to wall volume was found to be the most important geometrical determinant for average myofiber stress and strain (3). In a more detailed mathe-

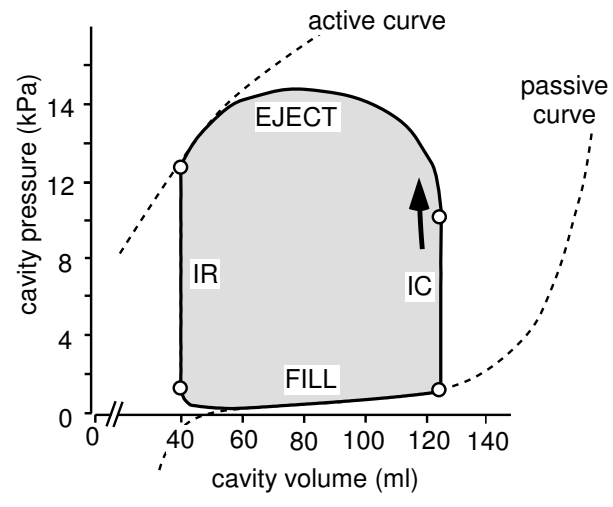

A

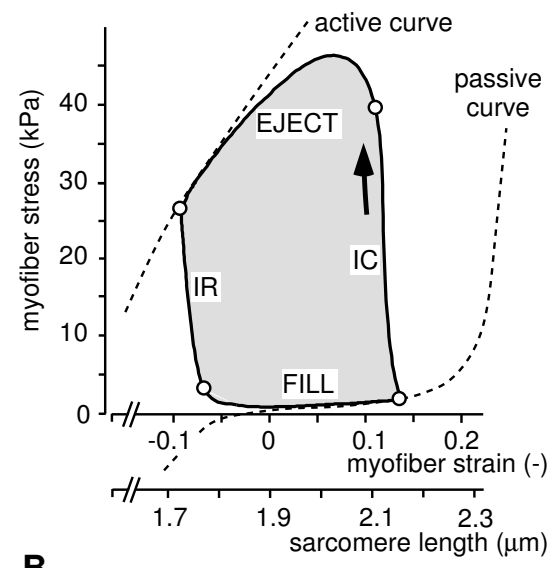

B

Figure 1.2. Quantification of mechanical load during the cardiac cycle by A LV cavity pressure as a function of volume at the global organ level, and by B myofiber stress as a function of myofiber strain at the local tissue level. The cardiac cycle consists of four phases: FILL=filling phase; IC=isovolumic contraction phase; EJECT=ejection phase; $\mathrm{IR}=$ isovolumic relaxation phase; $o=$ valve opening/closing. the pressure-volume and myofiber stress-strain loops are bounded by two curves describing the passive and active behavior of the myocardium. The arrows indicate the direction in which changes occur in time. 
matical analysis, it was furthermore found that spatial variation of myofiber stress and strain across the cardiac wall is highly sensitive to the orientations of the myofibers $(11,76)$, i.e., variation in orientations within the range of experimental data resulted in significant differences in stress and strain.

\subsection{MECHANICALLY INDUCED GROWTH AND REMODELING}

In the short-term, neurohumoral control ensures that blood pressure and flow generated by the heart as a whole are matched to the needs of the body (Fig 1.3). For instance, a low systemic blood pressure as sensed by specialized baroreceptors induces, among other responses, an increase in cardiac contractility (baro-reflex). Consequently, cardiac pump force increases and blood pressure is normalized (43).

Although an increase in contractility enhances cardiac output to match the needs of the body, tissue workload also increases with respect to normal. In the long-term, an elevated mechanical load has been shown to induce growth and remodeling (G\&R) of the tissue. Thus, while neurohumoral control attempts to match global cardiac output to the needs of the body, G\&R attempts to normalize local tissue loads. Unfortunately, much remains unknown about the relation between mechanical stimuli and responses involved in G\&R. For instance, there is no consensus about the type of mechanical loading (e.g., stress or strain) that triggers $G \& R(34,38,50)$. Furthermore, it is not yet fully understood how these stimuli transduce into changes in tissue composition and structure or how these changes affect cardiac output. Also, the adaptive capacity of the cardiac cells and the extent through which G\&R can restore normal mechanical load are unknown.

\subsubsection{Experimental models}

Most of current knowledge on the transduction of local mechanical stimuli to local tissue responses (mechano-transduction) is derived from in vitro cell culture and in vivo animal experiments. These experimental models mainly focus on unraveling the molecular aspects of tissue G\&R. In vitro models are especially valuable to investigate the relation between mechanical load, the resulting alterations in gene-expression, and the molecular signaling pathways involved. For instance, it was found that stretching of cardiac cells in vitro is sufficient to alter gene-expression and stimulate protein synthesis $(10,29,57,61-63,66,79)$. Stretch was also found to reduce protein degradation significantly $(13,59)$. In vivo studies are well-suited to investigate changes in, e.g., gene expression and tissue mass in relation to global changes in hemodynamic load. For instance, in vivo changes in workload during embryonic development were found to affect cardiac gene-expression, shape, size and structure $(32,46,67,68,73)$. Furthermore, 
in the adult heart, increased hemodynamic load results in an increase of wall mass in an attempt to normalize tissue load $(20,24,33,34,50)$. The associated change in myocyte shape depends on the type of overload, i.e., in case of pressure overload cells predominantly thicken, whereas in case of volume overload cells predominantly lengthen $(27,56,81)$. In addition to changes in tissue mass, changes in material properties such as stiffness are involved as well $(24,67)$.

While these experimental approaches have contributed significantly to our understanding of mechanically induced G\&R in the heart, the precise quantification of mechanical load (e.g., stress or strain), their related tissue response, and the effect on pump capacity remains poorly understood. This is mainly due to limitations of the applied experimental methodology. First, in vitro models have their limitations in describing the in vivo situation. For instance, the mechanical environment of the cells is different, i.e., deformation in in vitro experiments is usually restricted to uniaxial or biaxial extension $(44,45,58)$, while the three-dimensional deformation pattern in vivo is more complex (52). Second, it is hard to quantitatively extend results of in vitro gene-expression to local changes in tissue structure and to changes in cardiac output. Third, local quantitative information on amplitude and distribution of the mechanical stimulus as well as on the resulting tissue response is difficult to obtain in vivo. Tissue stresses in particular cannot be measured reliably in vivo (36).

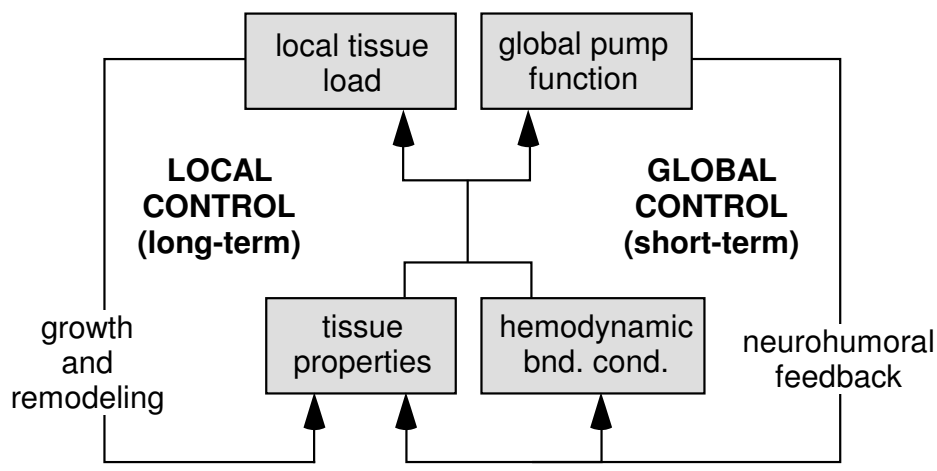

Figure 1.3. Conceptual model of short-term and long-term regulation of cardiac tissue properties, mechanical load and pump function. Cardiac pump function and mechanical load are determined by the properties of the cardiac tissue (e.g., stiffness, structure, and geometry) and the hemodynamic boundary conditions as imposed by the vasculature (e.g., vascular resistance). In the short-term, global control through neurohumoral feedback modulates cardiac tissue properties to match pumping action of the heart as a whole to the needs of the body. However, as a consequence of global control, local mechanical load of the tissue (e.g., stress and strain) may no longer be at its normal value. In the long-term, successful growth and remodeling of the tissue leads to normalization of mechanical load. 


\subsubsection{Mathematical models}

Parallel to in vitro and in vivo research on the molecular aspects of G\&R, (biomedical) engineers have studied the mechanical aspects of normal cardiac physiology $(1,2,5,20-24,28,34,37,51,53-55,68-70,78)$. Using mathematical models based on elementary laws of physics such as balance of force, local tissue composition and structure as well as global organ shape can be quantitatively related to local mechanical load and to function of the organ as a whole. Consequently, mathematical models provide the possibility to quantitatively link local tissue scale and global organ scale, thereby overcoming limitations of in vitro and in vivo experimental models. An additional advantage of mathematical models is that stress, which cannot be measured accurately in experimental models, is readily available.

Traditionally, mathematical models are applied to study the forward relation from cardiac tissue properties to local mechanical load and pump function $(4,6,9,11$, $16,42,49,64,71,77)$. In an attempt to unravel the role of stress and strain in cardiac G\&R, these forward models were applied in an inverse procedure to compute local stress and strain retrospectively from experimentally measured time series of parameters such as mass, cavity volume, and cavity pressure $(20,24,34)$. Such an approach allows evolution of stress and strain to be correlated with the evolution of the G\&R response. The accuracy of the model computations, however, is limited by the accuracy at which its input parameters are experimentally measured. In particular, it has been questioned whether current techniques to measure myofiber orientations are sufficiently accurate (26). In light of the previously mentioned high sensitivity of mechanical load distribution to the myofiber orientation, a high accuracy in myofiber orientations is required to correctly estimate mechanical load. Also, forward models do not include mechanical feedback during the G\&R process. As such, their ability to predict the long-term outcome of tissue G\&R effects on cardiac pump capacity and function is limited.

More recently, forward models have been combined with so-called adaptation rules or evolution laws that phenomenologically describe the local feedback from mechanical load to changes in tissue properties (Fig 1.3). These models have been applied successfully to predict the $G \& R$ responses in load-bearing tissues $(2,17,21-23,28,35,68,78,80)$.

In our group, we have developed similar models to study G\&R in actively contracting cardiac tissue $(4,5)$. Motivated by the apparent influence of mechanical load on cardiac gene-expression (32), myofiber orientations (73), and morphology $(46,53,67)$ during embryogenesis, we consider the healthy heart as a natural G\&R experiment. Consequently, hypotheses on local stimulus-response relations in cardiac G\&R have been tested on their ability to produce a realistic (healthy) normal LV (Fig 1.4). Indeed, with a mathematical model of mechanically induced G\&R, Arts et al. 1994 could estimate realistic values for cavity volume, wall vol- 
ume, and myofiber orientations (5). However, in their forward model of cardiac mechanics, the heart was geometrically simplified to a rotationally symmetric cylinder that allows for transmural variation in mechanics and tissue properties only (one-dimensional model). As such, that model is unsuitable to study inhomogeneous G\&R that may occur during disease (e.g., after a local infarction) or treatment (e.g., during artificial cardiac pacing).

\subsection{AIMS AND OUTLINE OF THE THESIS}

In this thesis we aim to gain additional insights into the stimulus-response relations in cardiac G\&R using two- and three-dimensional mathematical models which are capable of simulating complex G\&R patterns. Since tissue volume and the pattern of myofiber orientations have been identified as the key determinants of mechanical tissue load, volumetric growth and myofiber reorientation are the primary G\&R responses studied. Analogous to Arts et al. 1994, the general approach is to translate hypothesized stimulus-response relations into adaptation laws (5). By combining these adaptation laws with a forward model of cardiac mechanics, G\&R is simulated over time. Agreement of the model-estimates with experimental data would then support the hypotheses.
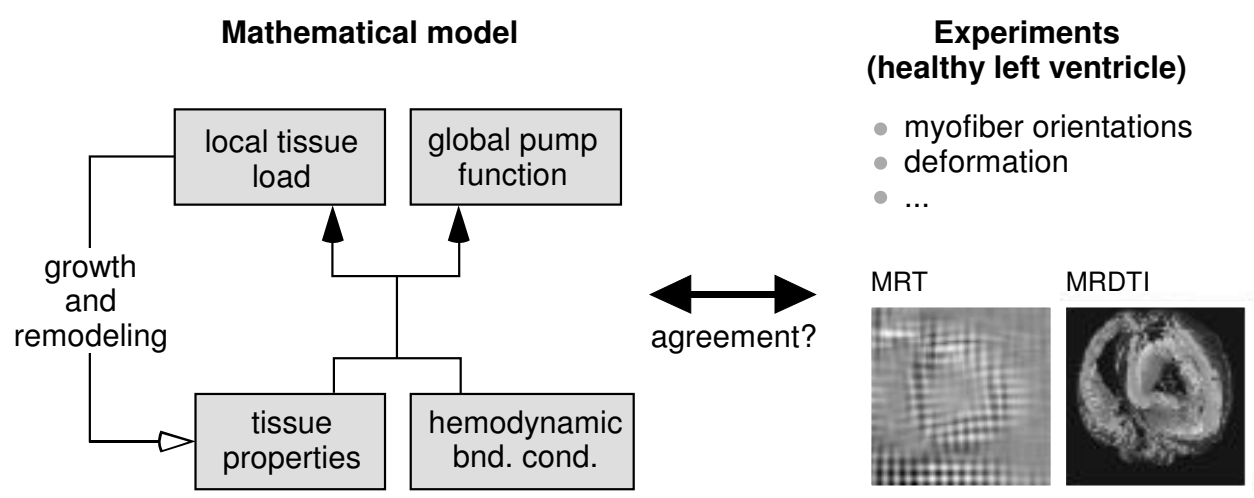

Figure 1.4. Approach as adopted in this thesis to study mechanically induced cardiac G\&R with mathematical models. In the mathematical model, forward relations from local tissue properties to local mechanical load and global pump function are captured by a model of cardiac mechanics ( $\longrightarrow$ ). The relation between mechanical load and growth and remodeling is accounted for by adaptation rules ( $\longrightarrow$ ). The model is evaluated by comparing model estimates in tissue deformation and structure with data on the healthy left ventricle as determined from, e.g., magnetic resonance tagging (MRT) and magnetic resonance diffusion tensor imaging (MRDTI) experiments. 
Our research is focussed on the healthy left ventricle (LV). Besides the normal $\mathrm{LV}$, we also consider the LV in the very rare case of situs inversus totalis (SIT) ${ }^{\dagger}$. Function of the SIT heart appears normal, however, limited histological data suggest myofiber orientations are not $(7,48,72)$. Since the SIT heart consists of the same type of cells as the normal heart, the same stimulus-response relations should be applicable to both hearts. As such, the aberrant structure of the SIT LV provides for an additional possibility to evaluate our hypotheses regarding myofiber reorientation.

Direct assessment of cardiac myofiber orientations in SIT via magnetic resonance diffusion tensor imaging (MRDTI) is hampered by the limited availability of SIT hearts ex vivo. The pattern of myofiber orientations, however, is reflected in the deformation of the wall. In particular, shear strains such as torsion were found to be sensitive to the myofiber orientations $(12,76)$. In chapter 2 , we present a study in which LV torsion in SIT is measured in vivo using magnetic resonance tagging (MRT). The normal LV is considered as control.

Subsequently, in chapter 3, we develop a 2D cylindrical shell model of mechanically induced growth and myofiber reorientation, and investigate which stimulusresponse relations in growth and myofiber reorientation yield a realistic geometry, structure, and torsion for both the normal and the SIT LV.

Next, to relieve restrictions on geometry, material description and deformation still present in the 2D shell model, a 3D finite element (FE) model is used to study G\&R in more detail. In chapter 4, a study is presented in which we investigate the hypothesis that the 3D pattern of myofiber orientations is determined by myocardial shear deformation. Myofiber orientations as estimated in the FE model are compared to the orientation pattern as obtained from MRDTI experiments. Subsequently, in chapter 5, we investigate to what extent reorientation of the myofibers yields a closer agreement between torsion and circumferential-radial shear strains in the model and those as obtained from MRT experiments.

Similar to chapter 3, we would like to include volumetric growth as an additional adaptation response in the FE model. However, especially in the complex case of $3 \mathrm{D}$ inhomogeneous volumetric growth, mathematical modeling is not straightforward. In chapter 6, we present a FE model with which we can simulate loadinduced 3D inhomogeneous volumetric growth.

This thesis concludes with a general discussion in chapter $\mathbf{7}$ in which the findings of the various chapters are put into a broader perspective.

${ }^{\dagger}$ Footnote: In SIT, internal anatomy is completely left-right mirror-imaged in the sagittal plane. Hence, instead of the normal position of the heart on the left side of the body, the heart is now located on the right side. Prevalence is about 1 in every 10.000 humans. 


\section{REFERENCES}

1. Alastrue V, Pena EA, Martinez MA, Doblare M (2007) Assessing the use of the "opening angle method" to enforce residual stresses in patient-specific arteries. Ann Biomed Eng 35:18211837.

2. Alford PW, Humphrey JD, Taber LA (2007) Growth and remodeling in a thick-walled artery model: effects of spatial variations in wall constituents. Biomechan Model Mechanobiol 7:245262.

3. Arts T, Bovendeerd PH, Prinzen FW, Reneman RS (1991) Relation between left ventricular cavity pressure and volume and systolic fiber stress and strain in the wall. Biophys $\mathrm{J}$ 59:93102.

4. Arts T, Delhaas T, Bovendeerd P, Verbeek X, Prinzen FW (2005) Adaptation to mechanical load determines shape and properties of heart and circulation: the CircAdapt model. Am J Physiol Heart Circ Physiol 288:H1943-H1954.

5. Arts T, Prinzen FW, Snoeckx LH, Rijcken JM, Reneman RS (1994) Adaptation of cardiac structure by mechanical feedback in the environment of the cell: a model study. Biophys $\mathrm{J}$ 66:953961.

6. Arts T, Reneman RS, Veenstra PC (1979) A model of the mechanics of the left ventricle. Ann Biomech Eng 7:299-318.

7. Asami I, Koizumi K (1989) [the vortex cordis is never reversely directed, even in situs inversus and L-loop anomaly] Kaibogaku Zasshi 64:36-45.

8. Baicu CF, Stroud JD, Livesay VA, Hapke E, Holder J, Spinale FG, Zile MR (2003) Changes in extracellular collagen matrix alter myocardial systolic performance. Am J Physiol Heart Circ Physiol 284:H122-H132.

9. Beyar R, Sideman S (1984) A computer study of the left ventricular performance based on fiber structure, sarcomere dynamics, and transmural electrical propagation velocity. Circ Res 55:358-375.

10. Borg TK, Terracio L (1990) Interaction of the extra-cellular matrix with myocytes during development and disease. In: Robinson TR, Kinne RK (Eds) Cardiac myocyte-connective tissue interactions in health and disease, Karger Basel, pp. 113-130 (ISBN-13: 978-3-8055-5028-4).

11. Bovendeerd PH, Arts T, Huyghe JM, van Campen DH, Reneman RS (1992) Dependence of local left ventricular wall mechancis on myocardial myofiber orientation: a model study. J Biomech 25:1129-1135.

12. Bovendeerd PH, Huyghe JM, Arts T, van Campen DH, Reneman RS (1994) Influence of endocardial-epicardial crossover of muscle fibers on left ventricular wall mechanics. J Biomech 27:941-951.

13. Byron KL, Puglisi JL, Holda JR, Eble D, Samarel AM (1996) Myosin heavy chain turnover in cultured neonatal rat heart cells: effects of $\left[\mathrm{ca}^{2+}\right]_{i}$ and contractile activity. Am J Physiol Cell Physiol 271:C1447-C1456.

14. Caulfield JB, Borg TK (1979) The collagen network of the heart. Lab Invest 40:364-372.

15. Cleutjens JP, Kandala JC, Guarda E, Guntaka RV, Weber KT (1995) Regulation of collagen degradation in the rat myocardium after infarction. J Mol Cell Cardiol 27:1281-1292.

16. Costa KD, Holmes JW, McCulloch AD (2001) Modeling cardiac mechanical properties in three dimensions. Phil Trans R Soc Lond A 359:1233-1250.

17. Cowin SC (1993) Bone stress adaptation models. J Biomech Eng 115:528-533.

18. de Tombe PP, ter Keurs HE (1990) Force and velocity of sarcomere shortening in trabeculae from rat heart. effects of temperature. Circ Res 66:1239-1254.

19. Dokos S, Smaill BH, Young AA, LeGrice IJ (2002) Shear properties of passive ventricular myocardium. Am J Physiol Heart Circ Physiol 283:H2650-H2659. 


\section{Chapter 1}

20. Donker DW, Volders PG, Arts T, Bekkers BC, Hofstra L, Spätjens RL, Beekman JD, Borgers M, Crijns HJ, Vos MA (2005) End-diastolic myofiber stress and ejection strain increase with ventricular volume overload: Serial in-vivo analyses in dogs with complete atrioventricular block. Basic Res Cardiol 100:372-382.

21. Driessen NJ, Boerboom RA, Huyghe JM, Bouten CV, Baaijens FP (2003) Computational analysis mechanically induced collagen remodeling in the aortic heart valve. J Biomech Eng 125:549-557.

22. Driessen NJ, Cox MA, Bouten CV, Baaijens FP (2008) Remodelling of the angular collagen fiber distribution in cardiovascular tissues. Biomech Model Mechanobiol 7(2):93-103.

23. Driessen NJ, Wilson W, Bouten CV, Baaijens FP (2004) A computational model for collagen fibre remodelling in the arterial wall. J Theor Biol 226:53-64.

24. Emery JL, Omens JH (1997) Mechanical regulation of myocardial growth during volumeoverload hypertrophy in the rat. Am J Physiol Heart Circ Physiol 273:H1198-H1204.

25. Fagard $\mathrm{RH}$ (1997) Impact of different sports and training of cardiac structure and function. Cardiol Clin 15:397-412.

26. Geerts-Ossevoort L (2003) Cardiac myofiber reorientation: a mechanism for adaptation? Ph.D. thesis Eindhoven University of Technology, Eindhoven, The Netherlands.

27. Gerdes AM, Capasso JM (1995) Structural remodeling and mechanical dysfunction of cardiac myocytes in heart failure. J Mol Cell Cardiol 27:849-856.

28. Gleason RL Jr, Humphrey JD (2005) A 2D constrained mixture model for arterial adaptations to large changes in flow, pressure and axial stretch. Math Med Biol 22:347-369.

29. Goldsmith EC, Carver W, McFadden A, Goldsmith JG, Price RL, Sussman M, Lorell BH, Cooper G, Borg TK (2003) Integrin shedding as a mechanism of cellular adaptation during cardiac growth. Am J Physiol Heart Circ Physiol 284:H2227-H2234.

30. Granzier HL, Irving TC (1995) Passive tension in cardiac muscle: contribution of collagen, titin, microtubules, and intermediate filaments. Biophys J 68:1027-1044.

31. Greenbaum RA, Ho SY, Gibson DG, Becker AE, Anderson RH (1981) Left ventricular fibre architecture in man. Brit Heart J 45:248-263.

32. Groenendijk BC, Hierck BP, Vrolijk J, Baiker M, Pourquie MJ, Gittenberger-de Groot AC, Poelmann RE (2005) Changes in shear stress-related gene expression after experimentally altered venous return in the chicken embryo. Circ Res 96:1291-1298.

33. Grossman W (1980) Cardiac hypertrophy: useful adaptation or pathologic process? Am J Med 69:576-584.

34. Holmes JW (2004) Candidate mechanical stimuli for hypertrophy during volume overload. J Appl Physiol 97:1453-1460.

35. Huiskes R, Weinans H, Grootenboer HJ, Dalstra M, Fudala B, Slooff TJ (1987) Adaptive boneremodeling theory applied to prosthetic-design analysis. J Biomech 20:1135-1150.

36. Huisman RM, Elzinga G, Westerhof N, Sipkema P (1980) Measurement of left ventricular wall stress. Cardiovasc Res 14:142-153.

37. Humphrey JD (2001) Stress, strain and mechanotransduction in cells. J Biomech Eng 123:638641.

38. Humphrey JD, Rajagopal KR (2002) A constrained mixture model for growth and remodeling of soft tissues. Math Model Meth Appl Sci 12:407-430.

39. Hunter PJ, McCulloch AD, ter Keurs HE (1998) Modeling the mechanical properties of cardiac muscle. Prog Biophys Mol Biol 69:289-331.

40. Jugdutt $\mathrm{BI}$ (2003) Ventricular remodeling after infarction and the extracellular collagen matrix: when is enough enough? Circ 108:1395-1403.

41. Kentish JC, ter Keurs HE, Ricciardi L, Bucx JJ, Noble MI (1986) Comparison between the sarcomere length-force relations of intact and skinned trabeculae from rat right ventricle. Influence of calcium concentrations on these relations. Circ Res 58:755-768. 
42. Kerckhoffs RC, Bovendeerd PH, Kotte JC, Prinzen FW, Smits K, Arts T (2003) Homogeneity of cardiac contraction despite physiological asynchrony of depolarization: a model study. Ann Biomed Eng 31:536-547.

43. Lanfranchi PA, Somers VK (2002) Arterial baroreflex function and cardiovascular variability: interactions and implications. Am J Physiol Regul Integr Comp Physiol 283:R815-R826.

44. Lee AA, Delhaas T, McCulloch AD, Villarreal FJ (1999) Differential responses of adult cardiac fibroblasts to in vitro biaxial strain patterns. J Mol Cell Cardiol 31:1833-1843.

45. Lee EJ, Holmes JW, Costa KD (2008) Remodeling of engineered tissue anisotropy in response to altered loading conditions. Ann Biomed Eng 36:1322-1334.

46. Männer J (2000) Cardiac looping in the chick embryo: a morphological review with special reference to terminological and biomechanical aspects of the looping process. Anat Rec 259:248262.

47. Marieb EN, Mallatt J, Wilhelm PB (2005) Human anatomy, fourth edition. Pearson Eductation Inc., San Fransisco.

48. Matsumura H, Aizawa Y, Kumaki K (1990) Myocardial architecture in situs inversus viscerum totalis. In: Clark EB, Takao A (Eds) Developmental cardiology: morphogenesis and function, Futura Pub Co, Mount Kisco, pp. 605-624 (ISBN-13: 978-0-879-93382-1).

49. Nash MP, Hunter PJ (2000) Computational mechanics of the heart: from tissue structure to ventricular function. J Elas 61:113-141.

50. Omens JH (1998) Stress and strain as regulators of myocardial growth. Prog Biophys Mol Biol 69:559-572.

51. Omens JH, Fung YC (1991) Residual strain in rat left ventricle. Circ Res 66:37-45.

52. Park J, Metaxas D, Axel L (1996) Analysis of left ventricular wall motion based on volumetric deformable models and MRI-SPAMM. Med Imag Anal 1:53-71.

53. Ramasubramanian A, Latacha KS, Benjamin JM, Voronov DA, Ravi A, Taber LA (2006) Computational model for early cardiac looping. Ann Biomed Eng 34:1655-1669.

54. Rodriguez EK, Hoger A, McCulloch AD (1994) Stress-dependent finite growth in soft elastic tissues. J Biomech 27:455-467.

55. Rodriguez J, Giocolea JM, Gabaldon F (2007) A volumetric model for growth of arterial walls with arbitrary geometry and loads. J Biomech 40:961-971.

56. Russell B, Motlach D, Ashley WW (2000) Form follows function: how muscle shape is regulated by work. J Appl Physiol 88:1127-1132.

57. Sadoshima J, Izumo S (1997) The cellular and molecular response of cardiac myocytes to mechanical stress. Ann Rev Physiol 59:551-571.

58. Sadoshima J, Jahn L, Takahashi T, Kulik TJ, Izumo S (1992) Molecular characterization of the stretch-induced adaptation of cultured cardiac cells: an in vitro model of load-induced cardiac hypertrophy. J Biol Chem 267:10551-10560.

59. Samarel AM, Spragia ML, Maloney V, Kamal SA, Engelmann GL (1992) Contractile arrest accelerates myosin heavy chain degradation in neonatal rat heart cells. Am J Physiol Cell Physiol 263:C642-C652.

60. Schmid H, O'Callaghan P, Nash MP, Lin W, LeGrice IJ, Smaill BH, Young AA, Hunter PJ (2008) Myocardial material parameter estimation: a nonhomogeneous finite element study from simple shear tests. Biomechan Model Mechanobiol 7:161-173.

61. Serneri GG, Modesti PA, Boddi M, Cecioni I, Paniccia R, Coppo M, Galanti G, Simonetti I, Vanni S, Papa L, Bandinelli B, Migliorini A, Modesti A, Maccherini M, Sani G, Toscano M (1999) Cardiac growth factors in human hypertrophy. Relations with myocardial contractility and wall stress. Circ Res 85:57-67.

62. Simpson DG, Majeski M, Borg TK, Terracio L (1999) Regulation of cardiac myocyte protein turnover and myofibrillar structure in vitro by specific directions of stretch. Circ Res 85:e59e69. 


\section{Chapter 1}

63. Spinale FG (2007) Myocardial matrix remodeling and the matrix metalloproteinases: influence on cardiac form and function. Physiol Rev 87:1285-1342.

64. Stevens C, Remme E, LeGrice IJ, Hunter P (2003) Ventricular mechanics in diastole: material parameter sensitivity. J Biomech 36:737-748.

65. Streeter DD Jr, Spotnitz HM, Platel DJ, Ross J, Sonnenblick EH (1969) Fiber orientation in the canine left ventricle during diastole and systole. Circ Res 24:339-347.

66. Swynghedauw B (1999) Molecular mechanisms of myocardial remodeling. Phys Rev 79:215262.

67. Taber LA (1995) Biomechanics of growth, remodeling, and morphogenesis. Appl Mechan Rev 48:487-545.

68. Taber LA (1998) Mechanical aspects of cardiac development. Prog Biophys Mol Biol 69:237255.

69. Taber LA, Humphrey JD (2001) Stress-modulated growth, residual stress, and vascular heterogeneity. J Biomech Eng 123:528-535.

70. Taber LA, Perucchio R (2000) Modeling heart development. J Elas 61:165-197.

71. Taber LA, Podszus WW (1997) A laminated shell model for the infarcted left ventricle. Int J Solids Struct 34:223-241.

72. Taussig HB (1926) The anatomy of the heart in two cases of situs transversus. Bull Johns Hopkins Hops 39(4):199-202.

73. Tobita K, Garrison JB, Liu LJ, Tinney JP, Keller BB (2005) Three-dimensional myofiber architecture of the embryonic left ventricle during normal development and altered mechanical loads. Anat Rec A Discov Mol Cell Evol Biol 283:193-201.

74. Torrent-Guasp F (1973) The cardiac muscle. Fundacin, Madrid.

75. Torrent-Guasp F, Kocica MJ, Corno AF, Komeda M, Carreras-Costa F, Flotats A, Cosin-Aguillar J, Wen H (2005) Towards new understanding of the heart structure and function. Eur J Cardiothorac Sur 27:191-201.

76. Ubbink SW, Bovendeerd PH, Delhaas T, Arts T, van de Vosse FN (2006) Towards model based analysis of cardiac MR tagging data: relation between left ventricular shear strain and myofiber orientation. Med Imag Anal 10:632-641.

77. Usyk TP, Mazhari R, McCulloch AD (2000) Effect of laminar orthotropic myofiber architecture on regional stress and strain in the canine left ventricle. J Elast 61:143-164.

78. Watton PN, Hill NA, Heil M (2004) A mathematical model for the growth of the abdominal aortic aneurysm. Biomechan Model Mechanobiol 3:98-113.

79. Weber KT, Sun Y, Tyagi SC, Cleutjens JP (1994) Collagen network of the myocardium: function, structural remodeling and regulatory mechanisms. J Mol Cell Cardiol 26:279-292.

80. Wilson W, Driessen NJ, van Donkelaar CC, Ito K (2006) Prediction of collagen orientation in articular cartilage by a collagen remodeling algorithm. Osteoarthr Cartil 14:1196-1202.

81. Zierhut W, Zimmer HG, Gerdes AM (1991) Effect of angiotensin converting enzyme inhibition on pressure-induced left ventricular hypertrophy in rats. Circ Res 69:609-617. 




\section{Structure and torsion of the normal and situs inversus totalis cardiac left ventricle \\ I. Experimental data in humans}

Tammo Delhaas ${ }^{1,2}$, Wilco Kroon ${ }^{2}$, Wim Decaluwe ${ }^{1}$, Mirjam Rubbens ${ }^{4}$, Peter Bovendeerd ${ }^{4}$, and Theo Arts $^{3}$

Department of ${ }^{1}$ Pediatrics, ${ }^{2}$ Physiology and ${ }^{3}$ Biophysics, Cardiovascular Research Institute Maastricht, University Hospital Maastricht, The Netherlands; and ${ }^{4}$ Department of Biomedical Technology, Eindhoven University of Technology, Eindhoven, The Netherlands.

Am J Physiol Heart Circ Physiol. 295:H197-H201, 2008 


\section{ABSTRACT}

In 1926, the famous American pediatric cardiologist, Dr. Helen B. Taussig, observed that in situs inversus totalis (SIT) main gross anatomical structures and the deep muscle bundles of the ventricles were a mirror image of the normal structure, while the direction of the superficial muscle bundles remained unchanged (Taussig HB (1926) Bull Johns Hopkins Hosp 39:199-202). She and we wondered about the implication of this observation for left ventricular (LV) deformation in SIT. We used magnetic resonance tagging to obtain information on LV deformation, rotation, and torsion from a series of tagged images in five evenly distributed, parallel, short-axis sections of the heart of nine controls and eight persons with SIT without other structural (cardiac) defect. In the controls, during ejection, the apex rotated counterclockwise with respect to the base, when looking from the apex. Furthermore, the base-to-apex gradient in rotation (torsion) was negative and similar at all axial levels of the LV. In SIT hearts, torsion was positive near the base, indicating mirrored myofiber orientations compared with the normal LV. Contrary to expectations, torsion in the apical regions of SIT LVs was as in normal ones, reflecting a normal internal myocardial architecture. The transition zone with zero torsion, found between the apex and base, suggests that the heart structure in SIT is essentially different from that in the normal heart. This provides a unique possibility to study regulatory mechanisms for myocardial fiber orientation and mechanical load, which has been dealt with in the companion paper by Kroon et al. 2008.

\subsection{INTRODUCTION}

In 1926, the famous American pediatric cardiologist, Dr. Helen B. Taussig, described in two cases of situs inversus totalis (SIT) that main gross anatomical structures and the deep muscle bundles of the ventricles were a mirror image of the normal heart, while the direction of the superficial muscle bundles remained unchanged (10). She also speculated as to the physiological significance of the muscle layer arrangement: "In view of the fact that the position of the deep muscle layers of the heart gives the mirror image of the normal constituting the preponderant contractile mass, the superficial layer being relatively thin by comparison, one would expect the systolic rotation about the anatomical axis of the transposed heart to be the mirror image of the normal" (10). The intriguing finding of Dr. Taussig remained unnoticed in cardiology, except for a limited number of studies from Japan in 1989 (2) and 1990 (8) that confirmed her original observation that the superficial layer of morphological left (LV) and right (RV) ventricles in SIT does not display the mirror image of that in situs solitus (SS = normal organ arrangement). Her speculation on rotation was never tested experimentally. 
In fact, the assumption that the inner layers dominate the outer ones is debatable, since the large radius of the latter layers adds to a high mass and moment arm. In 1926, observations on cardiac rotation could only be made during cardiac surgery. Nowadays, we can non-invasively estimate cardiac rotation and torsion by means of magnetic resonance (MR) tagging. Given the possibilities of the latter technique, we assessed whether the myofiber pattern within the LV wall in SIT leads to the same, although inverse, LV deformation pattern as in normal subjects.

\subsection{METHOD}

\subsubsection{Experimental subjects}

Nine healthy controls ( 1 female, 8 males; age 10-56 yr with a median of 30 yr) and eight persons with SIT (3 females, 5 males; age 8-70 yr with a median of $23 \mathrm{yr}$ ) were investigated. Six of the latter group had Kartagener syndrome, generally characterized by SIT, ciliary dyskinesia, variable impairment of fertility, and sinusitis with or without bronchiectasis with chronic cough. In our study, none of the SIT group had bronchiectasis or other structural (cardiac) defects or abnormalities. Subjects were included only if no evidence of preexisting cardiac disease or other significant coexisting illness was found in a clinical examination. Exclusion criteria included a history of hypertension, diabetes, ischemic or valvular heart disease, regular use of medication for cardiovascular illness, or a resting blood pressure above 160/90 $\mathrm{mmHg}$. On the 12-lead ECG, atrial fibrillation, bundle branch block, pathological Q-waves, LV hypertrophy, or changes consistent with myocardial ischemia resulted also in exclusion. The investigation conformed to the principles outlined in the Declaration of Helsinki. The Medical Ethics Committee of the University Hospital Maastricht approved the research protocol, and informed consent was obtained from all participants.

\subsubsection{Image acquisition and analysis}

MRI experiments were performed at 1.5 T (Gyroscan NT, Philips Medical Systems, Best, The Netherlands). Images were acquired in breath hold using ECG triggering, starting $20 \mathrm{~ms}$ after the R-wave. Using spatial modulation of the magnetization (3), two series of line-tagged images with lines in either horizontal (septal-lateral) or vertical (antero-posterior) direction were obtained over a period of $80-90 \%$ of the cardiac cycle in five evenly distributed, parallel, short-axis (SA) sections of the heart. Additionally, balanced fast-field echo (non-tagged) reference cine images were made. The following parameter settings were used: phase interval, $\sim 20 \mathrm{~ms}$; slice thickness, $8 \mathrm{~mm}$; intertag distance, $5 \mathrm{~mm}$; field of view, $200 \mathrm{~mm}$ for children and $250 \mathrm{~mm}$ for adults; image size, $256 \times 256$ pixels. 
MR images were analyzed off-line with custom software using Matlab 7.0 (MathWorks, Natick, MA) (11). The LV wall was manually outlined for each slice in a midsystolic cine image. This frame functions as reference for the displacement measurements to minimize errors due to large deformation. The tagged images were spatially band filtered around the line tag frequency (spatial frequency 0.14 $\mathrm{mm}^{-1}$, ratio of bandwidth to center frequency 1.0). The negative counterpart of the frequencies was filtered out. Applying two-dimensional inverse Fourier transformation to both filtered spectra, band filtered images resulted with a complex amplitude per pixel. Next, local phase and local frequency were derived. With the use of a correlation method previously applied for pulsed ultrasonic echo signals (4), vertical displacement maps for each time interval were calculated from the successive images with horizontal tag lines, whereas horizontal displacement maps were obtained from the images with vertical tag lines. Displacement per pixel was determined as the phase difference between both images, divided by the local spatial frequency, which has been determined as the local phase shift per pixel. Thus horizontal and vertical displacement maps were derived from pairs of vertically and horizontally tagged images, respectively.

Using the derived two-dimensional displacement information for each slice, rotation $(\Phi)$ of the LV wall was calculated as the average of rotational movement of each pixel within the wall about the centroid of the cavity. Cavity area $\left(A_{c, t}\right)$ was estimated as the sum of the manually outlined cavity area in the reference image and the change of this area relative to that in the outlined image. The end of the ejection phase was defined as the moment in time where $d A_{c, t} / d t$, averaged over all slices, approximated zero. Wall area $\left(A_{w}\right)$ was calculated in the reference frame as the area between the epi- and endocardial contours. For each section between subsequent slices, torsion $(\tau)$ was calculated as the axial gradient in rotation angle multiplied by the average of the outer radii $\left(r_{o}\right)$ of the upper $(u)$ and lower $(l)$ slice of that section:

$$
\tau=\frac{\Phi^{u}-\Phi^{l}}{d} \cdot \frac{r_{o}^{u}+r_{o}^{l}}{2} \quad \text { with } \quad r_{o}=\sqrt{\frac{A_{w}+A_{c, t}}{\pi}}
$$

with $d$ denoting the distance between the two SA cross sections. Physically, this measure of torsion may be interpreted as the negative of the circumferential-axial shear angle on the epicardial surface between the SA cross sections.

\subsection{RESULTS}

The balanced fast-field echo non-tagged cine images revealed normal cardiac anatomy and organ position in the control group, whereas these gross anatomy entities were mirror-imaged in SIT. 
For a 35-yr-old man with SS (=normal situation), rotation of five LV SA slices and torsion of the enclosed four sections are shown as a function of time in Fig 2.1A and Fig 2.1B, respectively. During practically the whole cardiac cycle, when viewing from the apex, the rotation angle remained positive for all five LV SA slices, indicating counterclockwise rotation with respect to the end-diastolic reference frame. Rotation rate is the first derivative of the rotation angle over time, i.e., the slope of the lines in Fig 2.1A. The sign of this rotation rate indicates the instantaneous direction of the rotation movement. Thus rotation of all slices

situs solitus

(normal)
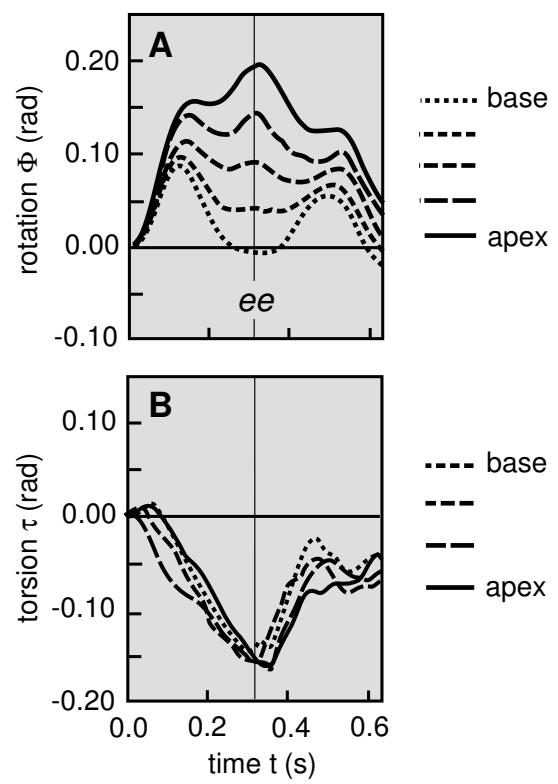

situs inversus totalis

(SIT)
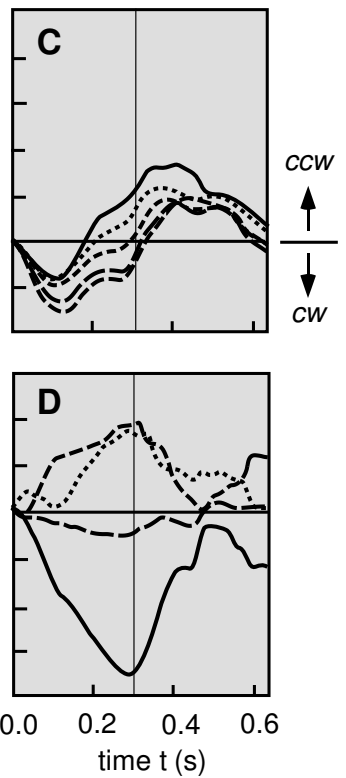

Figure 2.1. Time course of rotation angle and torsion of left ventricular (LV) short-axis slices in a normal subject and in one with situs inversus totalis (SIT). Positive rotation is counterclockwise when viewing the heart from the apex. Zero time corresponds to 20 ms after the R-wave. A and $\mathbf{B}$ : in the normal subject, the rotation angle at the end of the ejection phase increases gradually from base to apex. As a result, torsion, being proportional to the axial gradient in rotation angle, is about uniform during systole for all 4 enclosed sections. C and D: in the SIT, LV early-systolic rotation is opposite to normal. Thereafter, all slices rotate counterclockwise, albeit with different pace. At the end of the ejection phase, the apex rotated most counterclockwise just as the base did, although to a lesser degree. Net rotation of the midventricular slice is practically zero. Near the apex, torsion is about normal, whereas, near the base, torsion is inverted. As a transition, torsion for the zone between slices 3 and 4 is about zero. $c c w=$ counterclockwise; $c w=$ clockwise; $e e=$ end of the ejection phase. 
was counterclockwise in early-systole, reflecting a counterclockwise rotation of the whole heart. Thereafter, for the different slices, rotation diverges. The apical slice continues to rotate counterclockwise toward the end of the ejection phase, whereas the most basal slice rotates back in a clockwise direction. The slices in between apex and base show gradual transition patterns. At the end of the ejection phase, the rotation angle increases gradually from base to apex. As a result, torsion, being proportional with the axial gradient in rotation angle, is about uniform for all four sections during systole. The negative sign of torsion indicates that, looking from the apex, the LV apex rotates more counterclockwise than the LV base.

LV rotation and torsion patterns for a 30-yr-old man with SIT are shown in Fig 2.1C and Fig 2.1D. Early in systole, the SIT heart rotates inversely compared with the normal situation. All slices rotate clockwise, although the slices halfway

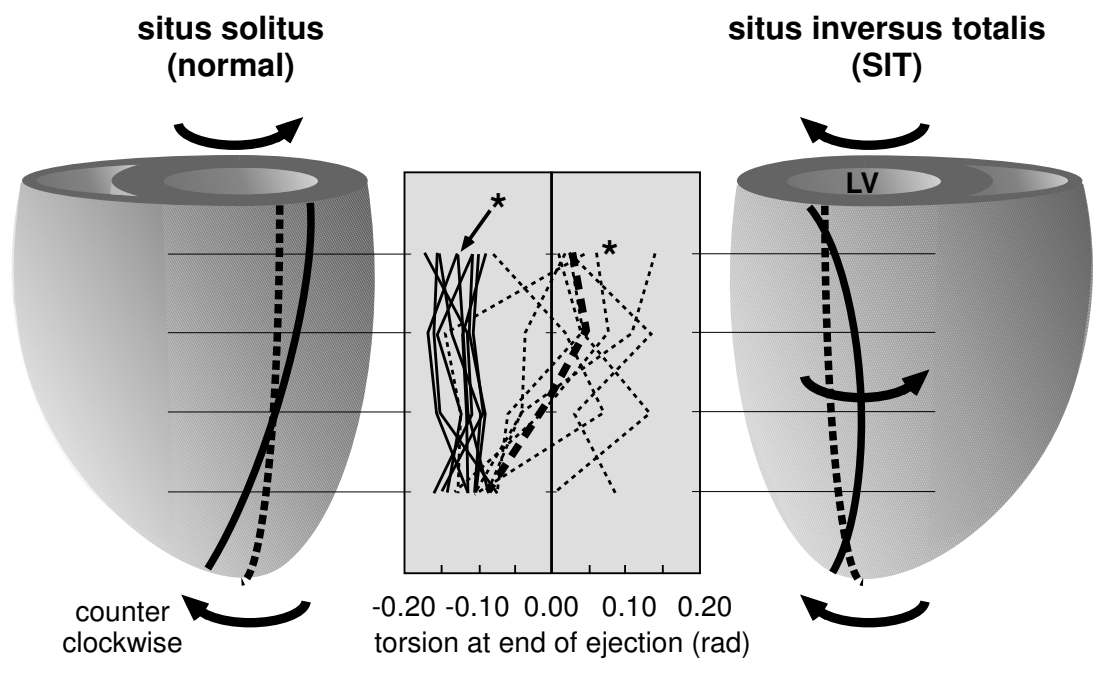

Figure 2.2. Systolic torsion of the LV in normal subjects and in those with SIT. Asterisks (*) indicate the subjects presented in more detail in Fig 2.1. In normal subjects ( - ), systolic LV torsion around the long axis is about uniform for all 4 sections in all subjects studied. In SIT ( ....-), systolic LV torsion is nonuniform with relatively large interindividual and intraventricular differences. The thick dashed line (-.-.) connects the mean values for each section. Near the apex, torsion is as in normal subjects. In the more basal sections, torsion gradually changes to an inverted torsion near the base. Frontal views are shown of the ventricular chambers in normal (left) and SIT (right) subjects. The dashed epicardial reference lines for beginning of ejection deform to the solid lines at the end of ejection. The normal LV, as viewed from the apex, exhibits a counterclockwise apical rotation with respect to the base. When viewed from the apex, apart from rigid body motion, LV motion in SIT can be described as having fixed both base and apex, while the midventricle rotates clockwise in between. 
between apex and base rotate most. Both apex and base rotate less and to about the same amount with respect to the reference frame. From midsystole, rotation becomes counterclockwise, but for all slices with a different pace. At the end of the ejection phase, the apex has the largest counterclockwise rotation angle with respect to the reference frame, followed by the slice near the base. At the end of the ejection phase, there is no net rotation of the midventricular slice, whereas the slices in between midventricle and apex are about equally rotated clockwise. Using the fact that torsion is proportional to the base-to-apex gradient of rotation, apical and basal torsion appear opposite. Near the base, torsion is inverted $\left(\Phi^{u}>\Phi^{l}\right)$, whereas, near the apex, torsion is about normal $\left(\Phi^{u}<\Phi^{l}\right)$. As a transition, torsion between the apex and the base is about zero $\left(\Phi^{u}=\Phi^{l}\right)$.

Fig 2.2 summarizes the results on torsion from the beginning to end of ejection, here called systolic torsion, of the subjects studied. The asterisks indicate the subjects presented in more detail in Fig 2.1. In the middle panel, LV systolic torsion is indicated for the four sections studied in normal subjects and in SIT. It clearly shows that in normal subjects the amount of LV systolic torsion is in a rather narrow range for all four sections in all subjects studied. Apart from rigid body motion, LV rotation is counterclockwise relative to the basal slice, as viewed from the apex. Systolic torsion is about uniform from apex to base in normal subjects, being $-0.13 \pm 0.03,-0.12 \pm 0.02,-0.13 \pm 0.02$, and $-0.13 \pm 0.03 \mathrm{rad}$.

In contrast, LV systolic torsion in SIT is nonuniform. Interindividual differences are rather large, and large differences are found between base and apex. Despite the interindividual differences, a common pattern can be observed, as depicted by the thick dashed line connecting the mean values of systolic torsion for each section. In the apical section, systolic torsion is in a normal direction, with amplitude somewhat lower than normal $(-0.08 \pm 0.05 \mathrm{rad})$. The following, more midventricular section exhibited hardly any systolic torsion $(-0.01 \pm 0.07 \mathrm{rad})$, whereas both the midventricular and basal sections have slight positive systolic torsion, being $0.02 \pm 0.08$ and $0.03 \pm 0.06 \mathrm{rad}$, respectively. Therefore, apart from rigid body motion, LV systolic motion in SIT can be described as having fixed both base and apex, while the midventricle rotates clockwise in between, as viewed from the apex.

\subsection{DISCUSSION}

Our results clearly show that, although gross anatomy is mirror-imaged in SIT, this is not the case for LV systolic deformation. Whereas LV torsion in normal subjects is negative and about uniform for all four sections studied, in SIT apical and basal torsion were opposite. Near the base, torsion is indeed inverted compared with normal subjects, but, near the apex, torsion has the same direction and almost the same amplitude as in normal subjects. 
Torsion is closely related to the distribution of myofiber orientation. In the normal SS heart, subepicardial fibers follow the path of a left-handed helix around the cavity, fibers in the midwall are circumferentially oriented, and subendocardial fibers follow a right-handed helical path $(5,9)$. Due to this specific pattern of fiber orientation, shortening of subendocardial fibers during systole tends to rotate the apex in a clockwise direction with respect to the base, while shortening of the subepicardial fibers tends to rotate the apex in the opposite direction. Since we measured a counterclockwise apical rotation with respect to the base, we conclude that the subepicardial fibers dominate the subendocardial ones in SS, as far as torsion is concerned. The negative torsion of the apical half of the LV in SIT is about normal, suggesting that the myocardial fiber orientation pattern is as in normal subjects. The positive torsion of the LV basal half in SIT suggests an inverted fiber orientation pattern in the basal region. In the midventricular sections with hardly any torsion, the pattern of fiber orientation is thought to be a mixture
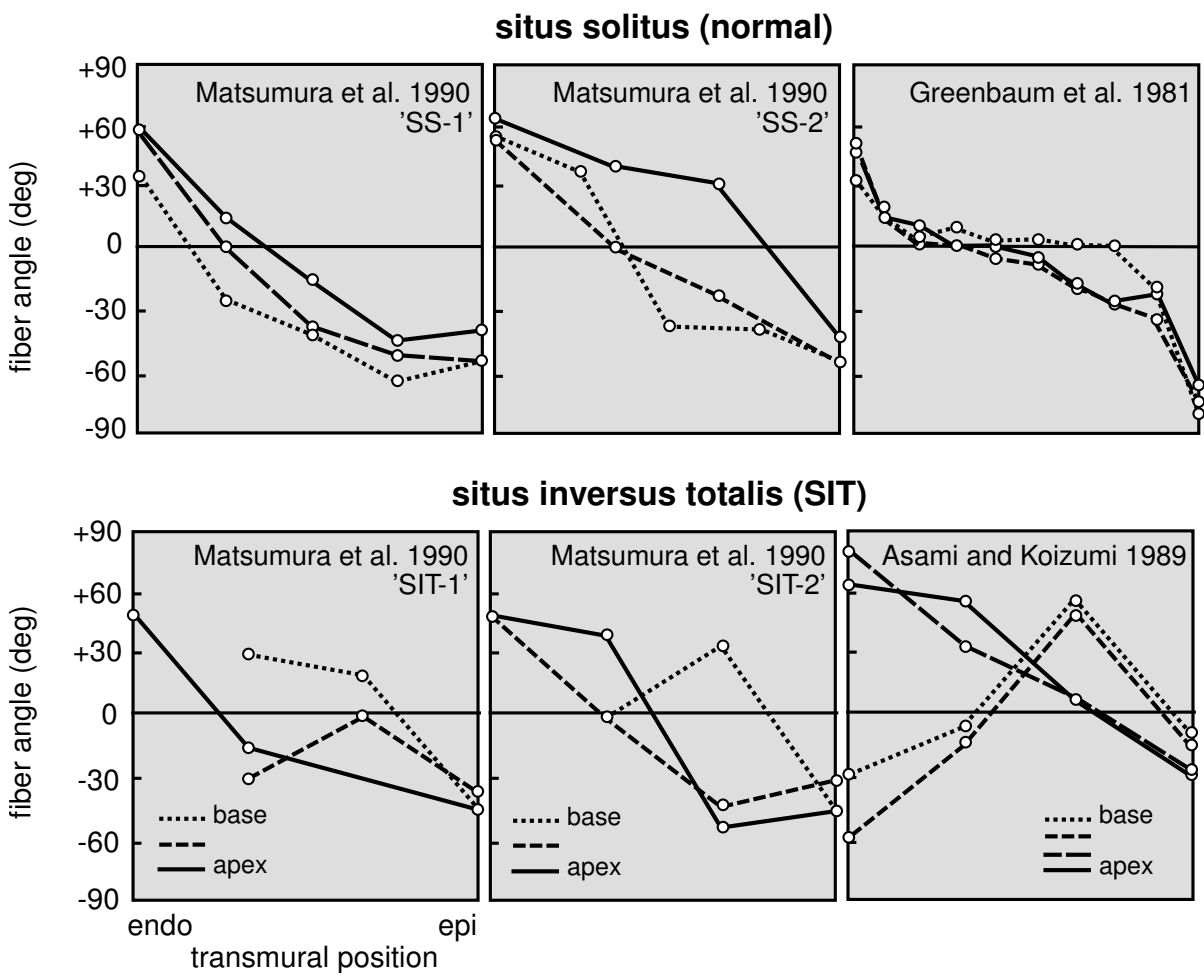

Figure 2.3. Transmural course of the fiber angle in the LV anterior wall in normal human hearts [situs solitus (SS); top] and in human SIT hearts (bottom). Data are derived from Asami and Koizumi 1989, Greenbaum et al. 1981, and Matsumura et al. 1990. 
of an inverted (basal type) inner half fiber pattern and a normal (apical type) outer half fiber pattern. The latter suggestion on the pattern of fiber orientation in SIT is supported by anatomical studies on LV myocardial fiber orientation in humans $(2,6,8,10)$. In Fig 2.3, we depict myocardial fiber angles in the LV anterior wall as derived from data in these studies ${ }^{\dagger}$. From these data, it can be appreciated that in normal hearts the pattern of the transmural change in fiber angle is qualitatively the same for the apex and the base of the LV. The SIT hearts, however, show a completely different pattern for the base and the apex. Whereas the apex of all SIT hearts studied shows the same pattern of transmural change in fiber angle as in the normal heart, the base of the SIT hearts has a partly mirror-imaged pattern of the transmural change in fiber angle. This difference is best appreciated in the SIT heart evaluated by Asami and Koizumi 1989. The myocardial fiber angle at the epicardium and the subepicardium of the anterior wall of the base of the SIT LV is as in the normal heart, whereas the deeper layers show an inverted pattern. It is to be noted that, although data regarding other areas than the anterior wall are not complete in the studies referenced in Fig 2.3, regional variation in the transmural course exists $(2,6,8)$. The abstract of Asami and Koizumi 1989 states: "The exception to this tendency (namely an inverted myofiber orientation pattern in the deeper layers of the basal levels) was found at the posterior region of the morphologically $L V$, in which there was no mirrorimaging but a normal pattern throughout the depth of the wall". This regional variation in fiber orientation pattern may also account for the differences in torsion pattern found within the SIT group (see below).

Ventricular torsion has been identified as a mechanism to reduce differences in fiber shortening within the LV wall. In the absence of torsion, during ejection, the amount of circumferential shortening, and hence fiber shortening, would be larger in subendocardial layers than in subepicardial layers. Torsion reduces subendocardial fiber shortening and amplifies subepicardial fiber shortening, leading to a more homogeneous distribution within the LV wall of fiber shortening during ejection (1). Mathematical model studies indicated that the specific helical myofiber pattern is needed to accommodate homogeneous fiber shortening. The specific fiber structure has been suggested to be the result of a myocardial adaptation process (1). In SIT, the LV rotation and torsion, as well as the helical myofiber pattern within the LV wall, are not a mirror image of the normal pattern: two poles of different functional (our study) and anatomical $(2,8,10)$ symmetry were found. Therefore, the SIT heart provides a unique possibility to study regulatory mechanisms for myocardial fiber orientation and mechanical load. This will be explored in detail in the accompanying computational study by Kroon et al. 2008.

${ }^{\dagger}$ Footnote: Data from Matsumura et al. 1990 are derived from their figure 1 (epicardial fiber angles in situs solitus), 4 and 5 (epicardial fiber angles in situs inversus totalis), and table 2 (fiber angles for the deeper layers in situs solitus and situs inversus totalis). Data from Asami and Koizumi 1989 are derived from their figure 4. Data from Greenbaum et al. 1981 are from their Fig 16, blocks 1-3 
Differences in the pattern of torsion between normal subjects and those with SIT cannot be explained by differences in heart rate variability or RV and/or LV loading conditions between the two groups. None of our subjects had conduction disturbances, myocardial infarct, or signs of (pulmonary) hypertension. In the normal heart, through-slice motion will not affect observed torsion, because the axial gradient in rotation angle is about uniform. In SIT, some differences in torsion may be attributed to through-slice motion, but it cannot explain the found dramatic difference between apical and basal torsion pattern. The variation in measured patterns of torsion in SIT suggests that every single SIT patient has a unique fiber orientation pattern, especially in the more basal parts of the LV. Deformation in these more basal parts of the LV influences, of course, the torsion pattern in the apical part. Also, regional variations in fiber orientation pattern, as well as transmural depth at which the normal fiber orientation pattern changes to an inverted one, influence the sign and amplitude of systolic torsion. Future, more detailed studies on the anatomy and deformation of both apical and basal slices might shed more light on the differences found for the torsion pattern within the SIT group.

Early systolic inverted rotation of the whole SIT heart is more likely to be due to the attachment of the heart by the large blood vessels than due to inversion of the internal myofiber structure near the base. After all, just after pulmonary valve opening, pressure in the pulmonary artery rises quickly, causing the RV outflow tract to increase in length and diameter. Consequently, the RV is pushed backward, causing whole heart rotation of the normal heart, as well as inverted rotation of the SIT heart.

In conclusion, although gross anatomy is mirror-imaged in SIT, the LV systolic deformation pattern is only partially mirror-imaged. In the normal heart, LV torsion is negative and quite uniform from base to apex. In SIT hearts, torsion is positive near the base, but negative near the apex. The sign of torsion reflects the handedness of the helical fiber pathways in the wall. In the SIT heart, mirrored torsion near the base reflects mirrored fiber structure. Contrary to expectations, near the apex torsion was as in normal subjects, reflecting a normal internal myocardial architecture. The transition zone with zero torsion between both types in myocardial architecture makes the SIT heart structure essentially different from the normal heart. Therefore, the SIT heart provides a unique possibility to study regulatory mechanisms for myocardial fiber orientation and mechanical load (see companion paper by Kroon et al. 2008).

\section{ACKNOWLEDGEMENTS}

This work was supported by The Netherlands Heart Foundation Grant 2000T036 and University Hospital Maastricht Grant PF155. T. Delhaas is a Clinical Fellow of The Netherlands Heart Foundation (Dr. E. Dekker Fund). 


\section{REFERENCES}

1. Arts T, Bovendeerd PH, Prinzen FW, Reneman RS (1991) Relation between left ventricular cavity pressure and volume and systolic fiber stress and strain in the wall. Biophys J 59:93102.

2. Asami I, Koizumi K (1989) [the vortex cordis is never reversely directed, even in situs inversus and L-loop anomaly] Kaibogaku Zasshi 64:36-45.

3. Axel L, Dougherty L (1989) MR imaging of motion with spatial modulation of magnetization. Am J Physiol Heart Circ Physiol 171:841-845.

4. de Jong PG, Arts T, Hoeks AP, Reneman RS (1990) Determination of tissue motion velocity by correlation interpolation of pulsed ultrasonic echo signals. Ultrason Imag 12:84-98.

5. Geerts L, Bovendeerd P, Nicolay K, Arts T (2002) Characterization of the normal cardiac myofiber field in goat measured with MR-diffusion tensor imaging. Am J Physiol Heart Circ Physiol 283:H139-H145.

6. Greenbaum RA, Ho SY, Gibson DG, Becker AE, Anderson RH (1981) Left ventricular fibre architecture in man. Brit Heart J 45:248-263.

7. Kroon W, Delhaas T, Bovendeerd P, Arts T (2008) Structure and torsion in the normal and situs inversus cardiac left ventricle. II. Modeling cardiac adaptation to mechanical loading. Am J Physiol Heart Circ Physiol 295:H202-H210.

8. Matsumura H, Aizawa Y, Kumaki K (1990) Myocardial architecture in situs inversus viscerum totalis. In: Clark EB, Takao A (Eds) Developmental cardiology: morphogenesis and function, Futura Pub Co, Mount Kisco, pp. 605-624 (ISBN-13: 978-0-879-93382-1).

9. Streeter DD Jr, Hanna WT (1973) Engineering mechanics for successive states in canine left ventricular myocardium. II. Fiber angle and sarcomere length. Circ Res 33:656-664.

10. Taussig HB (1926) The anatomy of the heart in two cases of situs transversus. Bull Johns Hopkins Hops 39(4):199-202.

11. van der Toorn A, Barenbrug P, Snoep G, van der Veen FH, Delhaas T, Prinzen FW, Maessen $\mathrm{J}$, Arts T (2002) Transmural gradients of cardiac myofiber shortening in aortic valve stenosis patients using MRI tagging. Am J Physiol Heart Circ Physiol 283:H1609-H1615. 



\section{Structure and torsion of the normal and situs inversus totalis cardiac left ventricle}

II. Modeling cardiac adaptation to mechanical load

Wilco Kroon ${ }^{2}$, Tammo Delhaas ${ }^{1,2}$, Peter Bovendeerd ${ }^{4}$, and Theo Arts $^{3}$

Department of ${ }^{1}$ Pediatrics, ${ }^{2}$ Physiology and ${ }^{3}$ Biophysics, Cardiovascular Research Institute Maastricht, University Hospital Maastricht, The Netherlands; and ${ }^{4}$ Department of Biomedical Technology, Eindhoven University of Technology, Eindhoven, The Netherlands.

Am J Physiol Heart Circ Physiol. 295:H202-H210, 2008 


\section{ABSTRACT}

Mathematical models provide a suitable platform to test hypotheses on the relation between local mechanical stimuli and responses to cardiac structure and geometry. In the present model study, we tested hypothesized mechanical stimuli and responses in cardiac adaptation to mechanical load on their ability to estimate a realistic myocardial structure of the normal and situs inversus totalis (SIT) left ventricle (LV). In a cylindrical model of the LV, 1) mass was adapted in response to myofiber strain at begin of ejection and to global contractility (average systolic pressure), 2) cavity volume was adapted in response to myofiber strain during ejection, and 3) myofiber orientations were adapted in response to myofiber strain during ejection and to local misalignment between neighboring tissue parts. The model was able to generate a realistic normal LV geometry and structure. In addition, the model was also able to simulate the instigating situation in the rare SIT LV with opposite torsion and transmural courses in myofiber direction between the apex and base (Delhaas et al. 2008). These results substantiate the importance of mechanical load in the formation and maintenance of cardiac structure and geometry. Furthermore, in the model, adapted myocardial architecture was found to be insensitive to myofiber misalignment in the transmural direction, i.e., myofiber strain during ejection was sufficient to generate a realistic transmural variation in myofiber orientation. In addition, the model estimates that, despite differences in structure, global pump work per unit of mass of the normal and SIT LV is similar.

\subsection{INTRODUCTION}

Mathematical models provide a suitable platform to test hypotheses on the relation between local mechanical stimuli and responses to cardiac structure and geometry, such as changes in mass $(18,22)$, shape $(23)$, and internal myofiber arrangement $(1,10,17,26)$. Model-based prediction of a realistic myocardial structure and geometry could substantiate the role of hypothesized stimuli and responses in real physiology.

Through mechanical feedback in the environment of the cell, Arts et al. 1994 were able to estimate a realistic normal left ventricular (LV) structure and geometry, i.e., myofiber orientations changed gradually across the wall from a subendocardial right-handed helix to a subepicardial left-handed one. In the normal $\mathrm{LV}$, there is little change in the transmural variation in myofiber orientation from apex to base. As a consequence, torsion in the normal LV is about equal at all levels from apex to base (6). As shown by Delhaas et al. 2008, the situs inversus totalis (SIT) LV, on average, exhibits a dualistic torsion pattern, with torsion at the apex being as in the normal LV, but opposite in sign at the base. The myocardial structure is dualistic as well, being the same as in the normal heart at the apex but mirrored at the base (Fig 3.3A, obtained from Matsumura et al. 1990). 
The aberrant nature of the myocardial structure in SIT provides an additional possibility to further test hypotheses regarding the mechanisms through which the cardiac wall adapts to mechanical load. In the present study, we investigated whether the hypotheses on adaptation used by Arts et al. 1994 are also able to generate the myocardial torsion and structure of the SIT LV (6). Special focus was on the transition zone between the apex and base.

To be able to incorporate the apex-to-base (axial) changes in myofiber orientation as present in the SIT LV, the one-dimensional model by Arts et al. 1994 was extended to include structural variations in the axial direction. Model-predicted LV structure and torsion were compared with experimental data reported by Delhaas et al. 2008. In a sensitivity study, the effect of the degree of myofiber coupling in the transmural and axial directions on the final cardiac structure and function was determined.

\subsection{METHOD}

In a numerical model, we simulated adaptation of global LV geometry and myofiber architecture to local mechanical tissue load. Mechanical quantities at begin and end of ejection as computed with a model of LV mechanics act as stimuli for adaptation of tissue mass, tissue shape (cavity volume), and myofiber orientations. Subsequently, the adapted myocardial structure and geometry are used in the end-diastolic state of the next cardiac cycle, thus completing an adaptation cycle. The process of adaptation was simulated as a sequence of adaptation cycles.

\subsubsection{Model of LV Mechanics}

Kinematics The LV geometry is represented by a rotational symmetric cylinder. To allow for axial variation in mechanics and structure, the cylindrical model is divided in a number of ring-shaped wall segments $\left(N_{\text {ring }}\right)$ stacked from the apex to base (Fig 3.1). Deformation of such a segment is assumed to be a combination of axial stretch $\lambda_{z}$, torsion $\tau$ (the negative of circumferential-axial shear strain), and contraction as quantified by the ejection volume $V_{e j}$.

As a consequence of the deformation field, a reference point with cylindrical coordinates $\left(r_{0}, \phi_{0}, z_{0}\right)$ translates to point $(r, \phi, z)$ :

$$
r=f\left(r_{0}, r_{i, 0}, V_{c a v, 0}, V_{e j}, \lambda_{z}\right) ; \quad z=\lambda_{z} z_{0} ; \quad \phi=\phi_{0}-\tau \frac{z}{r}
$$

where $V_{c a v, 0}$ and $r_{i, 0}$ are the cavity volume and internal radius in the reference configuration, respectively. Ejection volume $V_{e j}$ is prescribed. For a derivation of function $f$, we refer to the appendix. 
Constitutive laws The myocardial tissue is considered to be incompressible and to consist of myofibers with variable orientation embedded in an isotropic soft tissue matrix. Total Cauchy stress $\sigma$ in the tissue is considered to be the sum of hydrostatic pressure $p$ and the Cauchy stress $\sigma_{f}$ actively generated by the myofibers. In the ejection phase passive tissue stresses are neglected. Consequently, $\sigma$ is given by:

$$
\boldsymbol{\sigma}=-p \boldsymbol{I}+\sigma_{f} \vec{e}_{f} \vec{e}_{f}
$$

with $I$ the identity tensor. We define the myofiber direction $\vec{e}_{f}$ by the angle between the circumferential direction $\vec{e}_{\phi}$ and the axial direction $\vec{e}_{z}$, as follows:

$$
\vec{e}_{f}=\cos (\alpha) \vec{e}_{\phi}+\sin (\alpha) \vec{e}_{z}
$$

The relation between deformed myofiber angle $\alpha$ and reference myofiber angle $\alpha_{0}$ is given in the appendix. The active stress $\sigma_{f}$ generated by the myofibers during ejection depends linearly on their stretch ratio $\lambda_{f}$, elastance $E$, and normalized contractility $C$, as follows:

$$
\sigma_{f}=\left(\lambda_{f}-\lambda_{f, z s}\right) E C ; \quad \lambda_{f}=\frac{l_{f}}{l_{f, 0}}
$$

where $\lambda_{f, z s}$ represents the myofiber stretch ratio at zero myofiber stress, $l_{f}$ is the actual myofiber length, and $l_{f, 0}$ is a reference myofiber length. Given the reference geometry and myofiber angle $\alpha_{0}$, myofiber stretch $\lambda_{f}$ is derived as a function of ejection volume $V_{e j}$, torsion $\tau$, and axial stretch ratio $\lambda_{z}$ (see the appendix).

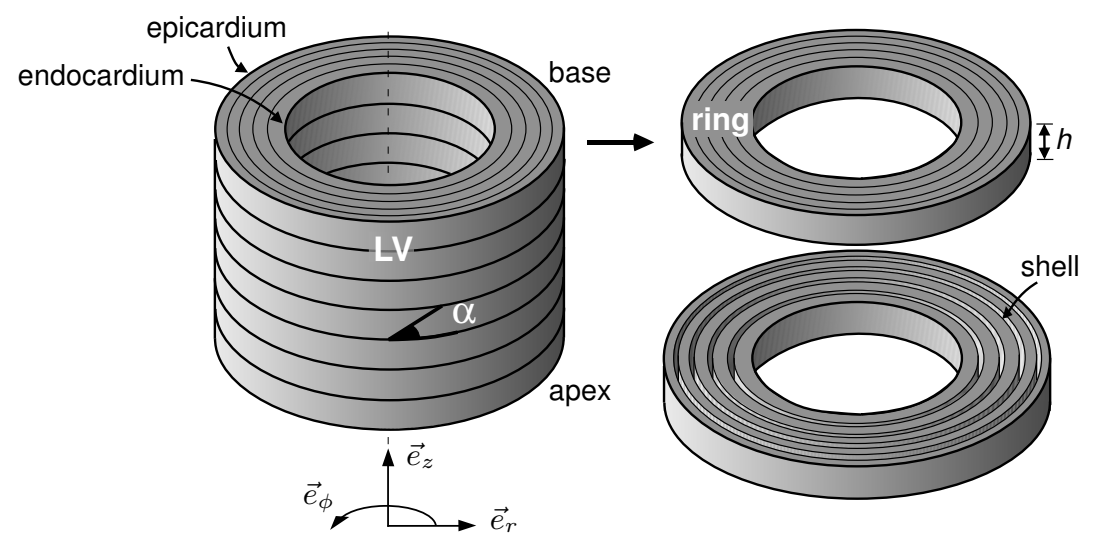

Figure 3.1. Cylindrical model of the left ventricle (LV). The LV wall is axially divided into ring segments with height $h$. Subsequently, each ring is divided into concentric shells with equal volume. The LV is attributed a cylindrical coordinate system $\left(\vec{e}_{r}, \vec{e}_{\phi}, \vec{e}_{z}\right)$. Myofiber directions are defined by angle $\alpha$ with respect to the circumferential direction $\left(\vec{e}_{\phi}\right)$ 
Normalized contractility $C$ of the myofibers represents the relative change in elastance $E$ required to reach an ejection pressure $\left(p_{c a v, e j, p r e f}\right)$ that is assumed to be preferred by the body. A value of $C=1$ means that $E$ is sufficient to generate an ejection cavity pressure that is equal to the preferred pressure.

Balance laws Within the wall, stresses are restricted by the balances of axial force, radial force, and axial momentum (e.g., Guccione et al. 1991). Assuming zero epicardial pressure:

$$
\begin{aligned}
\int_{r_{i}}^{r_{o}}\left[\sigma_{z z}-\frac{1}{2}\left(\sigma_{r r}-\sigma_{\phi \phi}\right)\right] r d r & =0 & & \text { Axial forces } \\
\int_{r_{i}}^{r_{o}} \sigma_{\phi z} r^{2} d r & =0 & & \text { Axial momentum } \\
\int_{r_{i}}^{r_{o}}\left[\frac{\sigma_{\phi \phi}-\sigma_{r r}}{r}\right] d r & =p_{\text {cav }} & & \text { Radial forces }
\end{aligned}
$$

where $p_{\text {cav }}$ is the cavity pressure and $r_{i}$ and $r_{o}$ are the inner and outer radii of the ring, respectively. The components of the stress tensor $\left(\sigma_{r r}, \sigma_{\phi \phi}, \sigma_{z z}\right.$, and $\left.\sigma_{\phi z}\right)$ are given by $\mathrm{Eq} 3.2$.

Numerical implementation Kinematics, constitutive laws, and balance laws are applied to each ring. Thus, each ring is attributed a torsion $\tau$ and an axial stretch $\lambda_{z}$. Ejection volume $V_{e j}$ of each ring is prescribed by the total ejection volume $V_{e j, t o t}$ of the LV, i.e., $V_{e j}=V_{e j, t o t} / N_{\text {ring }}$. The elastance $E$ is constant throughout the wall, indicating synchronous mechanical activity. The contractility $C$ is constant in each ring but may vary in the axial direction between ring segments.

Torsion $\tau$ and axial stretch $\lambda_{z}$ for the rings are iteratively determined from the balance laws in Eq 3.5 and Eq 3.6 by means of the conjugate gradient method for a given reference geometry and structure. To evaluate the integrals in Eq 3.5 through Eq 3.7, each ring is radially divided into a number of shells $\left(N_{s h}\right)$ that all have the same volume $V_{s h}$ (Fig 3.1). Consequently, the wall volume of the ring $V_{w}$ is given by $V_{w}=V_{s h} \times N_{s h}$. As a result, the continuous integrals over the radius $r$ become summations over the discrete number of shells (for details, see Arts et al. 1994). Myofiber stretch ratio $\lambda_{f}$ may vary between all shells in the transmural direction as well as in the axial direction.

The cardiac cycle Within the cardiac cycle, we discriminate between 1) end of diastole, which acts as the reference (as denoted by subscript 0 ), 2) begin of ejection (as denoted by subscript be), and 3 ) end of ejection (as denoted by subscript ee). During isovolumic contraction, the myofibers start to develop force as modeled by an increase in elastance $E$ to $80 \mathrm{kPa}$ at begin of ejection. Normalized contractility $C$ is set to 1 in all rings. During the transition from end of diastole to begin of ejection, LV cavity volume remains constant by specifying $V_{e j, t o t}=0$. 
To satisfy balance laws in Eq 3.5 and Eq 3.6, the individual rings will deform to their configuration at begin of ejection, exhibiting a torsion $\tau_{b e}$ and an axial stretch $\lambda_{z, b e}$. At the new equilibrium, Eq 3.7 gives the cavity pressures for each of the rings (denoted $\left.p_{c a v, b e}\right)$. During ejection, the volume change of the total LV $\left(V_{e j, t o t}\right)$ is set to $80 \mathrm{ml}$, whereas the elastance $E$ in all rings remains at $80 \mathrm{kPa}$. Again, balance laws in Eq 3.5 and Eq 3.6 provide the torsion $\tau_{e e}$ and axial stretch $\lambda_{z, e e}$, and Eq 3.7 gives the cavity pressure $p_{c a v, e e}$ for all rings at end of ejection.

Subsequently, contractility $C$ of the myofibers (Eq 3.4) in each ring adapts so that a preferred cavity pressure $p_{c a v, e j, p r e f}$ is reached. We relate the contractility to the mean of the LV cavity pressure at begin and end of ejection. Consequently, for ring $j$, the contractility is given by the following:

$$
C_{j}=\frac{p_{c a v, e j, p r e f}}{p_{c a v, e j, j}} ; \quad p_{c a v, e j, j}=\frac{p_{c a v, e e, j}-p_{c a v, b e, j}}{2}
$$

Next, a new cardiac cycle is simulated with the new contractility values $C_{j}$. The mechanical load quantities calculated at begin and end of ejection in this cycle are used as stimuli for adaptation.

\subsubsection{Rules for adaptation of geometry and myocardial archi- tecture}

Mechanical stimuli Wall mass, tissue shape (end-diastolic cavity volume), and myofiber orientations of each ring are locally adapted based on five load stimuli $s$ during the cardiac cycle. At begin of ejection, we define the myofiber stretch stimulus $s_{f, b e}$ for shell $i$ in $\operatorname{ring} j$ as follows:

$$
s_{f, b e, i, j}=\ln \left(\frac{\lambda_{f, b e, i, j}}{\lambda_{f, b e, p r e f}}\right)
$$

Similarly, we define stimuli due to deviations from preferred values of myofiber shortening during ejection $s_{f, e j}$ and normalized contractility $s_{C}$ :

$$
\begin{array}{rlrl}
s_{f, e j, i, j} & =\ln \left(\frac{\lambda_{f, e j, i, j}}{\lambda_{f, e j, p r e f}}\right) \quad \text { with } & \lambda_{f, e j, i, j}=\frac{\lambda_{f, e e, i, j}}{\lambda_{f, b e, i, j}} \\
s_{C, i, j} & =\ln \left(C_{j}\right) & \text { with } & C_{j}=\frac{p_{c a v, e j, p r e f}}{p_{c a v, e j, j}}
\end{array}
$$

In addition, we define stimuli at begin of ejection due to myofiber misalignment in transmural (radial) direction $s_{r}$ and axial direction $s_{z}$ :

$$
\begin{aligned}
& s_{r, i, j}=\left(\alpha_{b e, i, j}-\frac{\alpha_{b e, i+1, j}+\alpha_{b e, i-1, j}}{2}, \bmod \left(180^{\circ}\right)\right) \\
& s_{z, i, j}=\left(\alpha_{b e, i, j}-\frac{\alpha_{b e, i, j+1}+\alpha_{b e, i, j-1}}{2}, \bmod \left(180^{\circ}\right)\right)
\end{aligned}
$$


The modulus is added since angles at $+90^{\circ}$ and $-90^{\circ}$ essentially describe the same myofiber orientation.

It is noted that, during adaptation, sarcomere properties as described by Eq 3.4 are assumed to be unaffected.

Tissue mass Local tissue mass was considered to change in response to myofiber stretch ratio at begin of ejection $\lambda_{f, b e}$ and normalized contractility $C$ :

$$
g_{i, j}=s_{f, b e, i, j}+0.1 \cdot s_{C, i, j}
$$

where $g_{i, j}$ specifies the amount of growth. However, the calculation of mechanics requires a discrete number of shells with the same volume. Therefore, to implement growth, the shells were either duplicated and placed on the outside of the original shell (positive growth) or removed (negative growth). For this purpose, a probability function $G$ is computed from $g$;

$$
G_{i, j}=g_{i, j}+\delta_{g}
$$

The value of $\delta_{g}$, randomly taken between $-\beta$ and $+\beta$ (flat histogram), is added to translate a change of wall mass $g$ into a probability $G$ of shell duplication of removal. To duplicate or remove the shell;

$$
G_{i, j}=\left\{\begin{array}{cl}
>\beta & \text { positive growth, shell duplication } \\
<-\beta & \text { negative growth, shell removal }
\end{array}\right.
$$

We found that $\beta=0.5$ yielded a sufficiently fast and stable growth response.

Tissue shape In case myocardial tissue is subjected to large deformations as induced by increased filling, the cavity pressure-volume relationship has been shown to shift to higher volumes (8). Increased filling logically translates into increased end-diastolic myofiber strain. However, due to absence of the filling phase in our model, we use ejection myofiber strain as an estimate for enddiastolic strain. Consequently, to model the shift in the pressure-volume relation we change the end-diastolic ring cavity volume $V_{c a v, e d}$ before adaptation to $V_{c a v, e d}^{*}$ after adaptation, in response to the transmural average stimulus $s_{f, e j}$ :

$$
V_{c a v, e d, j}^{*}=V_{c a v, e d, j} \cdot \frac{1}{N_{s h}} \sum_{i=1}^{N_{s h}} \exp \left(s_{f, e j, i, j}\right)
$$

Each ring is assumed to contribute equally to the total end-diastolic cavity volume $V_{\text {cav }, e d}$, i.e., $V_{\text {cav }, e d}=V_{\text {cav }, e d, t o t} / N_{\text {ring }}$.

Myofiber orientation The end-diastolic (reference) myocardial structure, defined by angles $\alpha_{0, i, j}$, adapts via reorientation of the myofibers. We hypothesize that myofibers reorient to achieve a shortening during ejection of $15 \%$ 
$\left(\lambda_{f, e j, p r e f}=0.85\right)$. In addition, we hypothesize that myofibers reorient to form straight pathways in order to relieve internal stresses arising in the extra-cellular matrix due to myofiber contraction. Therefore, in the model, a local objective function $O_{i, j}$ was defined:

$$
O_{i, j}=s_{e j, i, j}^{2}+w_{r} s_{r, i, j}^{2}+w_{z} s_{z, i, j}^{2}
$$

The weight factors $w_{r}$ and $w_{z}$ scale contributions to $O_{i, j}$ of the misalignment in the transmural (radial) and axial direction, respectively. Higher values of $w_{r}$ and $w_{z}$ indicate a higher degree of myofiber alignment imposed on the myocardial structure.

To determine a more preferred myocardial structure, in each adaptation cycle, proposed end-diastolic (reference) myofiber angles $\alpha_{0}^{*}$ are determined by adding values $\delta_{\alpha}$ randomly selected between -2.5 and +2.5 degrees to the existing myofiber angles $\alpha_{0}$ :

$$
\alpha_{0, i, j}^{*}=\alpha_{0, i, j}+\delta_{\alpha}
$$

Next, a cardiac cycle is simulated with the proposed myofiber angles $\alpha_{0}^{*}$, and the values of the objective function $O^{*}$ are determined. The proposed angles are accepted if the corresponding value $O_{i, j}^{*}$ is less than the original value $O_{i, j}$. If not, myofiber angles remain unaltered.

\subsubsection{Simulations performed}

Adaptation in the single-ring model of the LV A multi-ring model of the LV requires boundary conditions at the apex and base. To obtain these boundary conditions, we first performed a set of simulations with a single ring, starting with random myofiber orientations. Total LV ejection volume was set at $80 \mathrm{ml}$. Initial cavity volume was arbitrarily set to $90 \mathrm{ml}$, and the wall initially consisted of 10 shells of $1 \mathrm{ml}$ each. Imposed myofiber alignment within the ring in the transmural direction was given by $w_{r}=1.0$. For a complete overview of the parameter values used in the model of cardiac mechanics and in the adaptation rules, see Table 3.1. Adaptation was simulated over 600 cycles.

In the simulations, after 600 cycles, either one of two stable myocardial structures was found (Fig 3.2A). In the NORM structure, myofiber angles gradually changed from positive at the endocardium to negative at the epicardium. The MIRROR structure was the mirror image of the NORM structure.

To asses the sensitivity of the final myocardial structure on $\lambda_{f, e j, p r e f}, p_{c a v, e j, p r e f}$, $E$, and $\lambda_{f, z s}$, a set of single-ring simulations was performed in which those parameters were individually increased and decreased. The resulting change of the myocardial structure was quantified by the root mean square (RMS) in the 
end-diastolic (reference) myofiber angle, as follows:

$$
R M S=\frac{1}{N_{s h}} \sum_{i=1}^{N_{s h}} \sqrt{\left(\alpha_{0, i}-\hat{\alpha}_{0, i}\right)^{2}}
$$

with $\alpha_{0}$ the angles that are obtained with the default values in Table 3.1 and $\hat{\alpha}_{0}$ the myofiber angles resulting from parameter change.

Adaptation in the multi-ring model of the LV Subsequently, adaptation is simulated with a model consisting of 30 axially stacked rings (the multi-ring model). Boundary conditions at the base and apex determined whether a normal LV or a SIT LV is simulated. For the normal LV, the NORM structure was used at the apical boundary as well as the basal boundary. For the SIT LV, the NORM structure was used at the apical boundary, whereas the MIRROR structure was taken at the basal boundary. Boundary structures remained unaltered during the adaptation process.

Between the apex and base, myofiber orientations, end-diastolic cavity volume, and wall volume were adapted. At the start, myofibers were randomly orientated while the end-diastolic cavity volumes for each ring were obtained by normalizing the resulting volumes in the single-ring simulation with respect to the number of rings. Shell volume was also normalized (equal to $1 / N_{\text {ring }}$; in $\mathrm{ml}$ ).

The imposed alignment between myofibers in the transmural and axial direction was specified by $w_{r}=1.0$ and $w_{z}=1.0$, respectively. The sensitivity of the final SIT structure on values of $w_{r}$ and $w_{z}$ was determined via simulations using combinations of threefold increased or threefold decreased values of $w_{r}$ and $w_{z}$.

$\begin{array}{llll}\text { Description } & \text { Symbol } & \text { Value } & \text { Unit } \\ \text { Initial total end-diastolic cavity volume } & V_{\text {cav, ed,tot,init }} & 90^{*} & \mathrm{ml} \\ \text { Initial number of shell per ring } & N_{\text {sh,init }} & 10^{*} & - \\ \text { Shell volume } & V_{\text {sh }} & 1^{*} \text { or } 1 / 30^{* *} & \mathrm{ml} \\ \text { Total ejection volume } & V_{\text {ej,tot }} & 80 & \mathrm{ml} \\ \text { Number of rings } & N_{\text {ring }} & 1^{*} \text { or } 30^{* *} & - \\ \text { Systolic myocardial elastance } & E & 80 & \mathrm{kPa} \\ \text { Myofiber stretch at zero stress } & \lambda_{f, z s} & 0.5 & - \\ \text { Preferred average systolic cavity pressure } & p_{\text {cav,ej,pref }} & 13 & \mathrm{kPa} \\ \text { Preferred myofiber stretch during ejection } & \lambda_{f, e j, p r e f} & 0.85 & - \\ \text { Preferred myofiber stretch at begin of ejection } & \lambda_{f, b e, p r e f} & 1.0 & - \\ \text { Apex-to-base alignment factor in myofiber reorientation } & w_{z} & 1.0^{* *} & - \\ \text { Transmural alignment factor in myofiber reorientation } & w_{r} & 1.0 & -\end{array}$

Table 3.1. Input parameters for the model of cardiac mechanics and for the adaptation rules in the simulation of cardiac adaptation to mechanical load. *Single-ring simulation. ${ }^{* *}$ Multi-ring simulation. 


\subsection{RESULTS}

\subsubsection{Adaptation in the single-ring model of the LV}

Fig 3.2 shows the resulting transmural course of end-diastolic (reference) myofiber angles and myofiber stretch ratios at begin and end of ejection for the singlering simulations.

In the NORM structure, the myofiber angle shows a gradual decrease from $+60^{\circ}$ at the subendocardium to about $-30^{\circ}$ at $92 \%$ of wall thickness, at which a large gradient occurs towards $-90^{\circ}$ at the subepicardium (Fig 3.2A). The MIRROR structure shows the mirrored transmural course from $-60^{\circ}$ at the subendocardium to about $+90^{\circ}$ at the subepicardium. Associated stretch ratios of the myofibers at begin and end of ejection for the NORM simulation as well as the MIRROR simulation have obtained their reference values in the major part of the wall except for a $2 \%$ deviation in shortening near $92 \%$ of the wall thickness. This is the transmural location where the large gradient in the myofiber angle occurs (Fig 3.2B).

Fig 3.2C through $\mathrm{E}$ show the evolution of average ejection pressure, wall volume, and end-diastolic cavity volume during the adaptation process, respectively. Table 3.2 shows that the global parameters in the single-ring LV have converged to stable values after 600 adaptation cycles. The obtained average ejection pres-

A

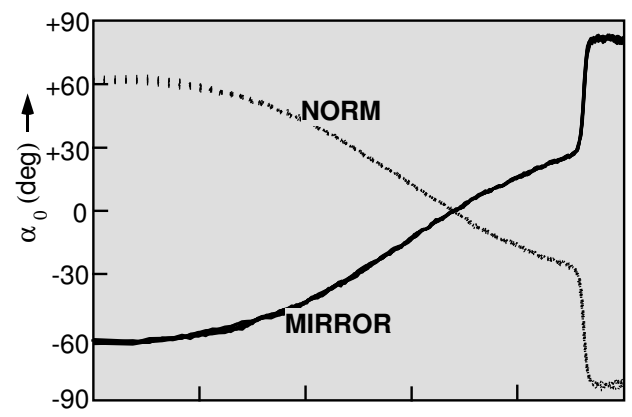

B

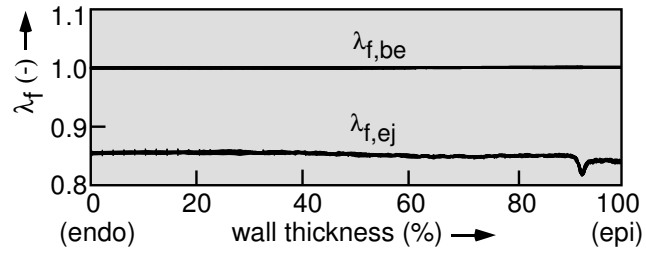

C

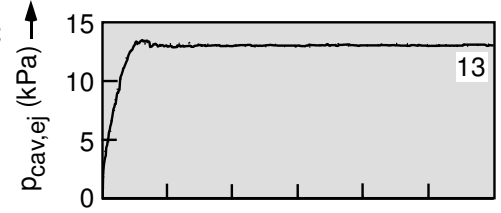

D

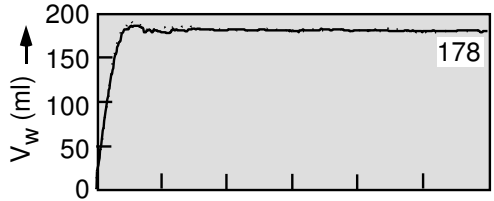

E

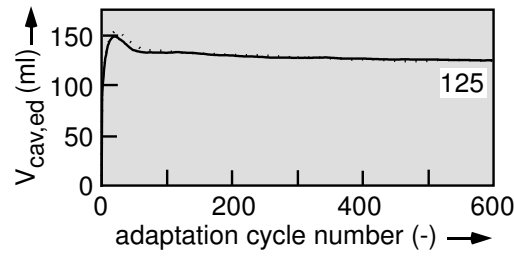

Figure 3.2. Results of adaptation in a single-ring for the NORM (broken line) and MIRROR simulation (solid line). A End-diastolic myofiber angle $\left(\alpha_{0}\right)$. B Myofiber stretch ratio at begin of ejection $\left(\lambda_{f, b e}\right)$ and during ejection $\left(\lambda_{f, e j}\right)$. C Average systolic cavity pressure $\left(p_{c a v, e j}\right)$. D Wall volume $\left(V_{w}\right)$. E End-diastolic cavity volume $\left(V_{c a v, e d}\right)$. A and B show results for the last 100 adaptation cycles in increments of 10 cycles. 
sure $p_{c a v, e j}$ is at the preferred level $p_{c a v, e j, p r e f}$ of $13 \mathrm{kPa}$, indicating that the increase in wall mass has fully compensated for the initial low ejection pressure (Fig 3.2C and D). Except for the sign of torsion at end of ejection, the adapted global parameters were not significantly different between the NORM and MIRROR simulations. Table 3.3 shows the RMS of the myofiber angles in response to changes in preferred myofiber stretch during ejection $\lambda_{f, e j, p r e f}$, preferred average ejection pressure $p_{c a v, e j, p r e f}$, myofiber elastance $E$, and myofiber stretch at zero stress $\lambda_{f, z s}$.

\subsubsection{Adaptation in the multi-ring model of the LV}

After 1,000 adaptation cycles, myofiber orientations in the multi-ring models of the normal and SIT LV have converged. For the multi-ring model of the normal LV, the resulting cavity volume, wall volume, torsion, and myofiber orientations were very similar to those obtained with the single-ring model in the NORM simulation (Table 3.2 and Fig 3.2 and Fig 3.3). Hence, for the analysis and discussion of the multi-ring simulations, we will focus on the SIT LV only.

The SIT LV developed a transition zone in between the normal orientations at the apex and the mirrored orientations at the base. In this transition zone, especially in the endocardial layers, more myofibers appear to have adopted an axial

\begin{tabular}{|c|c|c|c|c|c|}
\hline \multirow{2}{*}{ Parameter } & \multicolumn{2}{|c|}{ Single-ring simulation } & \multicolumn{2}{|c|}{ Multi-ring simulation } & \multirow{2}{*}{ Unit } \\
\hline & NORM & MIRROR & normal & SIT & \\
\hline End-diastolic cavity volume & $125.2 \pm 0.3$ & $125.0 \pm 0.3$ & $124.6 \pm 0.4$ & $118.1 \pm 0.3$ & $\mathrm{ml}$ \\
\hline Wall volume & $178.3 \pm 0.6$ & $178.0 \pm 0.7$ & $177.9 \pm 0.3$ & $177.7 \pm 0.3$ & $\mathrm{ml}$ \\
\hline Torsion at end of ejection & $-0.135 \pm 0.002$ & $+0.136 \pm 0.002$ & see Fig 3.3F & see Fig 3.3F & $\mathrm{rad}$ \\
\hline Ejection fraction & 63.7 & 63.8 & 64.2 & 69.4 & $\%$ \\
\hline
\end{tabular}

Table 3.2. Output parameters for the adapted LV. Simulation results are expressed as mean \pm standard deviation of results of the last 100 adaptation cycles. SIT=situs inversus totalis.

\begin{tabular}{lcccccccc} 
Parameter & \multicolumn{2}{c}{$\lambda_{f, \text { ej,pref }}$} & \multicolumn{2}{c}{$p_{\text {cav } \text { ej,pref }}$} & \multicolumn{2}{c}{$E$} & \multicolumn{2}{c}{$\lambda_{f, z s}$} \\
Value & 0.80 & 0.90 & $12 \mathrm{kPa}$ & $14 \mathrm{kPa}$ & $60 \mathrm{kPa}$ & $100 \mathrm{kPa}$ & 0.4 & 0.6 \\
\hline RMS (deg.) & 0.94 & 0.68 & 1.28 & 0.97 & 1.51 & 1.93 & 1.02 & 1.47
\end{tabular}

Table 3.3. Effect of a decrease and an increase of preferred myofiber stretch during ejection $\lambda_{f, e j, r e f}$, preferred average ejection pressure $p_{c a v, e j, p r e f}$, myofiber elastance $E$, and myofiber stretch at zero stress $\lambda_{f, z s}$ on the final myofiber angles. Changes in myofiber angles are expressed in root-mean-square (RMS) values. 
A

\section{normal}

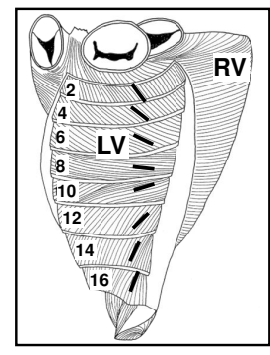

B

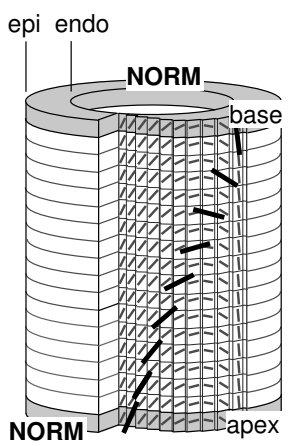

C

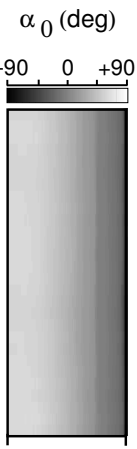

D

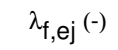

$\lambda_{\mathrm{f}, \mathrm{be}}{ }^{(-)}$

$\tau_{\text {ee }}(\mathrm{rad})$
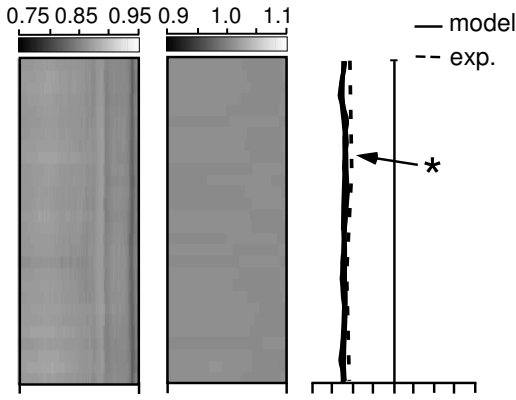

\section{situs inversus totalis}

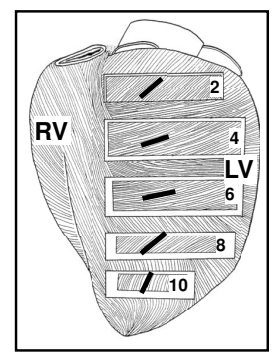

NORM

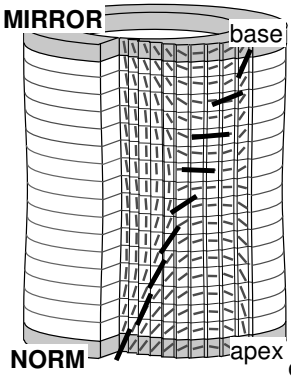

$$
\text { wall }
$$
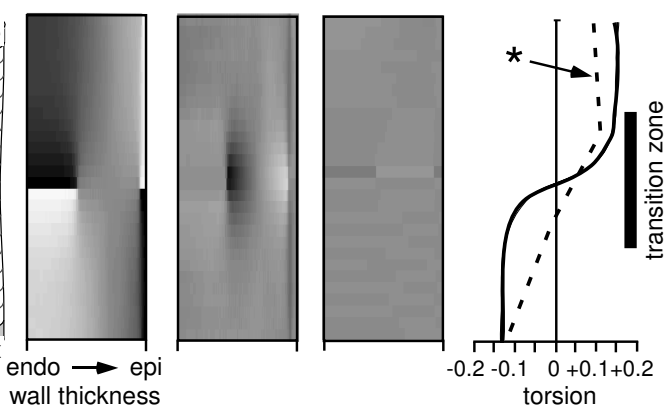

Figure 3.3. Real and model-predicted pattern of myofiber orientations in the normal and situs inversus totalis (SIT) LV. Model results were obtained after 1,000 adaptation cycles in a multi-ring model with 30 axially stacked ring segments. The figures show results interpolated to 18 rings consisting of 10 shells each. A Vector plots of experimentally determined myofiber orientations as adopted from Matsumura et al. 1990. Myofiber orientations at several transmural depths were obtained by peeling off layers of the myocardium. Numbers indicate the depth with respect to the outer layer. RV=right ventricle. B Vector plots of model-determined myofiber orientations. Boundary conditions were adopted in the shaded rings. For each axial level, myofibers were visualized at transmural depths that increase in circumferential direction. endo=endocardium; epi=epicardium. C Angle plots of myofiber orientations. Note that myofiber angles of $+90^{\circ}$ and $-90^{\circ}$ both refer to axially oriented myofibers. D Myofiber shortening during ejection $\left(\lambda_{f, e j}\right)$. E Myofiber shortening at begin of ejection $\left(\lambda_{f, b e}\right)$. $\mathbf{F}$ Torsion at the end of ejection $\left(\tau_{e e}\right)$. Solid lines are the model-determined torsion values for the last 100 adaptation cycles in increments of 10 . Dashed lines are experimental data from Delhaas et al. 2008 for the hearts indicated by the asterisks ( $*$ ). 
orientation. Fig 3.3E shows that the myofiber stretch ratio at begin of ejection $\left(\lambda_{f, b e}\right)$ is homogeneously distributed over the wall. However, within the transition zone, the myofiber stretch ratio during ejection $\left(\lambda_{f, e j}\right)$ is heterogeneously distributed, ranging from 0.75 to 0.95 (Fig 3.3D). After adaptation reached a stable result, cavity pressures within all rings of the SIT LV obtained a value of $13 \mathrm{kPa}$. Cavity and wall volume for the SIT LV were $118.1 \pm 0.3$ and $177.7 \pm 0.3$, respectively (Table 3.2). Torsion values at different levels between the apex and base are shown in Fig 3.3F. In the SIT LV, torsion gradually changed sign over the transition zone from $+0.136 \mathrm{rad}$ at the base to $-0.135 \mathrm{rad}$ at the apex.

Fig 3.4 shows the sensitivity of myofiber stretch ratios during ejection and torsion at end of ejection to different values of $w_{z}$ and $w_{r}$ for the SIT LV. With increasing axial coupling factor $w_{z}$ the transition zone widened, as reflected by the widening of the zone in which the myofiber stretch ratio during ejection is inhomogeneously distributed. Furthermore, torsion at the end of ejection changed more gradually from apex to base. Decreasing $w_{z}$ showed opposite results in myofiber shortening and torsion. Alterations in imposed myofiber coupling in the transmural direction by changing $w_{r}$ did not significantly affect myofiber stretch ratio and torsion patterns. Parameter $w_{r}$ could be reduced to zero without a significant change in myofiber stretch ratio and torsion patterns.

A transmural misalignment

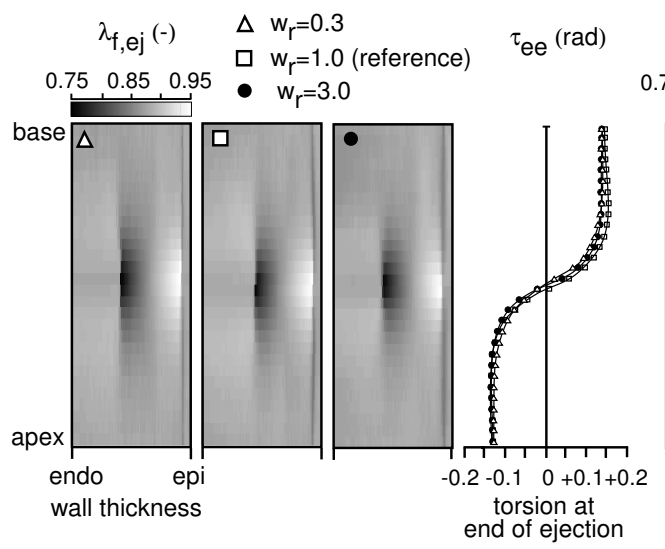

B axial misalignment

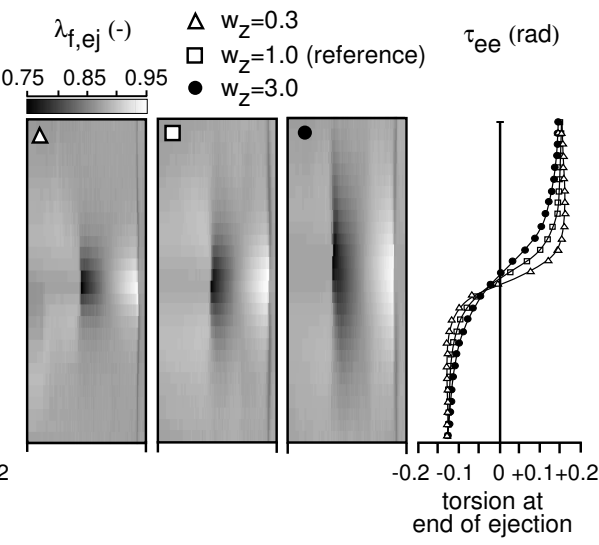

Figure 3.4. Resulting myofiber stretch ratio during ejection $\left(\lambda_{f, e j}\right)$ and torsion values at the end of ejection $\left(\tau_{e e}\right)$ for different values of scale factors in adaption $\left(w_{r}\right.$ and $\left.w_{z}\right)$. A Dependence on the amount of imposed myofiber alignment in the transmural direction $\left(w_{r}\right)$. B Dependence on the amount of imposed myofiber alignment in the apex-to-base direction $\left(w_{z}\right)$. 


\subsection{DISCUSSION}

In the present study, we investigate whether, based on adaptation to mechanical loading, we could estimate the myocardial torsion and structure of the SIT LV (6). We followed the approach as adopted by Arts et al. 1994 in which, in a model of cardiac mechanics, parameters of shape, mass, and internal myocardial architecture are simultaneously adapted based on mechanical stimuli. Stimuli were linked to adaptation responses of the tissue using adaptation rules that phenomenologically describe results from in vivo and in vitro experiments.

\subsubsection{Model results compared with experimental data}

First, similar to Arts et al. 1994, adaptation was simulated in a circumferentially symmetric model, representing a LV with no axial variation in myocardial structure (the single-ring model in the present study). As a result of adaptation, a random initial architecture converged to either one of two stable architectures that were mirror-imaged with respect to one another (the NORM and MIRROR myofiber angle distributions in Fig 3.2A). Geometrical parameters in both populations were identical (Table 3.2 and Fig 3.2C through E). End-diastolic volumes, ejection fractions, and wall mass were realistic at $\sim 125 \mathrm{ml}, \sim 64 \%$, and $\sim 178 \mathrm{ml}$, respectively. Myofiber shortening during ejection was homogeneous across the wall.

As shown in Fig 3.5, myofiber orientation in the NORM structure is within the range of experimental data of the normal LV. In addition, the torsion value of $+0.135 \mathrm{rad}$ at the end of ejection was in the range of experimental observations

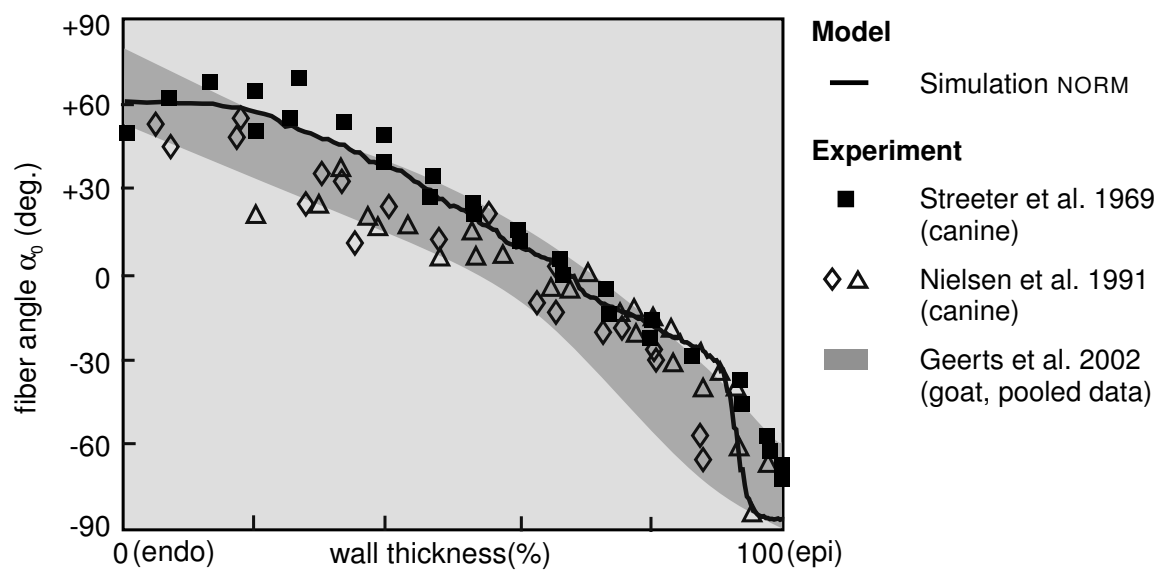

Figure 3.5. Comparison between the model-predicted transmural course in myofiber angle and experimental data for the normal LV. 
of the normal LV (6). The single-ring simulations typically show a large gradient in myofiber angle near the epicardium (Fig 3.2).

Furthermore shown in Fig 3.5 and also reported by Arts et al. 1994, experiments show a similar increase in transmural gradient in myofiber angle near the epicardium $(1,9,16,21)$. A possible explanation for this increased gradient has been provided previously (2). In short, near the epicardium, the myofiber direction is similar to the principle direction of tissue shortening (3). Consequently, the amount of shortening near the epicardium becomes relatively insensitive to the myofiber direction. Conversely, from the argument that the myofiber orientation adapts to achieve a certain amount of shortening, a wide range of myofiber directions are possible near the epicardium. Apparently, to achieve balance of forces in our model, this local degree of freedom in myofiber orientation results in a steep gradient to more axial orientations.

Our model suggests that the mirror image of the normal structure is stable as well. However, to the best of our knowledge, an LV with a completely mirrored structure has never been reported. Instead, the SIT LV was found to have a dualistic myocardial structure in which a normal structure at the apex appears to transit into a mirrored structure at the base (6) (Fig 3.3). To allow for the axial variation in the SIT myocardial structure, a multi-ring model was created. Preliminary simulations with the multi-ring model showed that when all rings were allowed to adapt, eventually the structure exhibited no transition zone, i.e., the structure became either fully normal (and equal to the results obtained with the single-ring model) or fully mirrored. Therefore, to enforce a transition zone, we fixed the ring at the basal boundary in the mirrored structure and the ring at the apical boundary in the normal structure as obtained after convergence in the single-ring model (Fig 3.2). Starting with a random intermediate structure, the local adaptation resulted in a stable geometry and structure (Table 3.2 and Fig 3.3). The final myocardial architecture exhibited a small transition zone between the apex and base with predominantly axially and circumferentially oriented myofibers (Fig 3.3B). Myofiber shortening during ejection was locally heterogeneous (Fig 3.3D). Currently, the experimental data presented or referred to by Delhaas et al. 2008 are all that is available on SIT hearts. Quantification of the SIT myocardial structure with magnetic resonance diffusion tensor imaging (MRDTI) could be used to assess in more detail whether the structure estimated by the model is realistic. However, existing SIT heart specimens have been preserved in formalin or ethanol-glycerine for a long time, which makes them unsuitable for MRDTI. Still, qualitatively, the model-estimated myofiber orientations shown in Fig 3.3B are similar to the experimental data shown in Fig 3.3A. Furthermore, the transmural course in myofiber angle is reflected by the torsion. Although there is some spread in the data, the experiments show a reversal in sign of torsion from apex to base, where absolute values of apical and basal torsion are similar. The torsion as estimated by the model exhibits the same characteristics (Fig 3.3F). 
The model shows some differences between the normal and SIT LV. Estimated end-diastolic cavity volume is slightly decreased by $5 \%$ with respect to that of the normal LV. Since stroke volume was used as a fixed input in the model, ejection fraction is somewhat elevated by $4 \%$ (Table 3.2). However, these differences are small. In addition, wall mass and ejection pressures were similar to those in the normal LV. This indicates that for both hearts, the globally generated external work (area pressure-volume loop) per unit of mass is equal, despite local heterogeneity in SIT myofiber shortening.

\subsubsection{Sensitivity analysis}

In the model, we used a preferred myofiber shortening and average cavity pressure during ejection as target values for the adaptation. Quantification of normal LV deformation with magnetic resonance tagging revealed myofiber shortening during ejection in the real normal LV to be $\sim 15 \%$ (14). In addition, cavity pressure during ejection is roughly $13 \mathrm{kPa}$. Apparently, in reality, the normal LV geometry and structure have adapted to achieve $\sim 15 \%$ myofiber shortening during ejection at an average pressure of $13 \mathrm{kPa}$. To investigate the sensitivity of the myocardial structure on the preferred values of myofiber shortening or ejection pressure, we performed a parameter variation in the single-ring model (Table 3.3). The simulations revealed that the RMS value in the end-diastolic myofiber angle was no more than $2^{\circ}$, indicating that changes in the myocardial structure were minor. Cavity volume, wall mass, and deformation (torsion and axial shortening) appeared to have adapted in such a way that the preferred myofiber shortening was achieved with the same myocardial structure.

In the multi-ring model, alignment of myofibers was imposed by coupling neighboring myofibers in the transmural and axial direction. To asses the influence of the degree of myofiber coupling on the final myofiber architecture, a parameter sensitivity analysis was conducted. A change in transmural myofiber coupling (factor $w_{r}$ ) from the reference value did not significantly alter final ejectional myofiber shortening and torsion (Fig 3.4A). In fact, even in the absence of coupling $\left(w_{r}=0\right)$, shortening and torsion did not change significantly. Since myofiber shortening is very sensitive to myofiber orientations (5), it is likely that the final myocardial structure did not change significantly as well. In contrast, shortening and torsion changed significantly in response to a variation in parameter $w_{z}$, controlling coupling between myofibers in the axial direction (Fig 3.4B). This indicates that myofiber orientations have changed as well. The size of the transition zone increased, as indicated by an increase of the zone in which myofiber shortening is heterogeneously distributed and by a more gradual change in torsion from the apical to basal value. Simulations with $w_{z}=0.3$ showed that no transition zone developed between the apical and basal structure, i.e., the structure in the upper half of the LV was fully mirrored, whereas the structure in the bottom half of 
the LV was fully normal. In case of $w_{z}=3.0$, the transition zone extended to the apical and basal boundaries. In that case, the prescribed apical and basal boundary conditions, rather than the adaptation rules, dominated the architecture in the transition zone. Our model suggests that for the development and maintenance of the transmural variation in myofiber orientation, imposed myofiber coupling in the transmural direction is not required. The mechanical load (myofiber shortening during ejection) sensed by each individual cell appears to be sufficient.

\subsubsection{Assumptions and limitations}

Adaptation rules The adaptation rules used in this study are based on the rules proposed by Arts et al. 1994. We added an axial component in the adaptation rules to study adaptation in SIT. In our model, 1) increased diastolic myofiber stretch and increased contractility are linked to an increase in wall mass, 2) increased strain excursions induces cavity dilation, and 3) myofibers align with their environment, attempting to achieve a preferred amount of systolic shortening. These rules are substantiated by more recent in vitro and in vivo data and remain up to date. For instance, strain remains one of the primary correlates to changes in wall mass and cavity volume $(7,8,11)$. Nonetheless, experiments have not yet ruled out other mechanical stimuli such as stress or strain rate $(12,22)$.

There is still some debate on whether increased contractility is a stimulus to hypertrophy. Cathecholamine release in response to low systemic pressure is known to induce an acute increase in contractility via adrenergic receptors, chronically leading to an increase in wall mass in vivo $(4,19)$. However, in an in vivo volume overload model, Holmes 2004 did not find a high correlation between observed contractility (as quantified by a maximum rate of pressure increase $\left.\left(d p / d t_{\max }\right)\right)$ and the increase in wall mass.

It is noted that, in the adult heart, myofiber reorientation was never confirmed in vivo or ex vivo (9). Therefore, it may seem odd that we propose myofiber reorientation as a means for the tissue to adapt to mechanical loading. However, the high sensitivity of load distribution to myofiber orientation (5) and the apparent absence of heterogeneities in loading in the real $\operatorname{LV}(25,27)$ advocate for the existence of a myofiber orientation-controlling mechanism (1). In other words, mechanical load redistribution may be achieved by a very small amount of myofiber reorientation. This amount may well fall within the measurement accuracy of $\sim 6^{\circ}$ for MRDTI, which is currently one of the most accurate techniques to measure myofiber orientations $(9,20)$. Thus, the lack of in vivo or ex vivo confirmation of myofiber reorientation does not exclude its existence.

In reality, adaptation is likely to be a continuous process. However, the time scale at which changes in geometry and structure occur are orders of magnitude larger than the time scale at which changes in mechanics occur during the cardiac cycle. We found that, provided the adaptation yielded a stable solution, this solu- 
tion was insensitive to the rate at which the adaptation occurs (see also Arts and Reneman 1988). Therefore, the time scale of the simulated adaptation becomes arbitrary. In our model, we have chosen to adapt the geometry and structure in a discrete fashion after each cardiac cycle to improve computational efficiency.

Mechanics model The presented mechanics model provides a suitable initial step toward simulation of load-induced adaptation. In this mechanics model, the cardiac LV is represented by a rotationally symmetric cylinder. The real LV basal geometry is, by good approximation, cylindrical, whereas the apical geometry is rather spherical. Also, the internal myocardial structure is not entirely symmetric, exhibiting markable differences between septum and free wall (e.g., Geerts et al. 2002). In addition, the right ventricle (RV) affects mechanics of the LV through direct mechanical coupling via the septum. Neglecting the contribution of the RV to LV mechanics may affect adaptation and, as a result, final geometry and structure of the LV. In future studies, finite element models may be used to include more complex asymmetries in cardiac geometry and structure to investigate the influence of the RV and a closed apex on the final structure.

Furthermore, the fluid-myofiber model of the cardiac tissue behavior during ejection neglects contributions of the passive matrix as well as transmural components in the myofiber orientation. During systole, stresses passively induced due to matrix deformation are usually much smaller than the actively developed stress (13). Therefore, exclusion of passive stress is not likely to affect the model results on myocardial structure and torsion. Similarly, although a transmural component in myofiber orientation may affect the torsion (24), the torsion pattern from the apex to base is not likely to be significantly affected. In the SIT LV model, a dualistic pattern will still be seen.

In addition, sarcomere elastance and myofiber stretch ratio at zero active stress did not change during adaptation. Experimental data are unclear as to whether sarcomere properties change during adaptation or not (22). The stress-strain relation, as given by Eq 3.4, was obtained from data presented by Donker et al. 2005. They found that during ejection, the stress-strain relation is approximately linear. Although variation of sarcomere elastance and myofiber stretch ratio at zero active stress resulted in a change in wall mass, cavity volume, and cavity pressure, they did not yield significant changes in myofiber orientation (Table 3.3).

\subsection{CONCLUSIONS}

In the present model study, we tested hypothesized mechanical stimuli and responses in cardiac adaptation to mechanical load on their ability to estimate a realistic myocardial structure of the normal and SIT LV. Local changes in tissue altered cavity volume, wall mass, and myocardial architecture at the organ level. 
The model was able to generate a realistic LV geometry and structure. In addition, the model was also able to simulate the instigating situation in the rare SIT LV with mirrored movements and transmural courses in myofiber direction between the apex and base (6). These results substantiate the importance of mechanical load in the formation and maintenance of cardiac structure and geometry. Furthermore, adapted myocardial architecture was found to be insensitive to myofiber misalignment in the transmural direction, i.e., myofiber strain during ejection was sufficient to generate a realistic transmural variation in myofiber orientation. In addition, the model estimates that the normal and SIT LV have similar global pump work and mass, despite differences in structure.

\section{APPENDIX}

As a consequence of deformation a material point displaces from cylindrical coordinates $\left(r_{0}, \phi_{0}, z_{0}\right)$ to coordinates $(r, \phi, z)$. Globally, the deformation is parameterized by a ejection volume $V_{e j}$, axial stretch $\lambda_{z}$ and torsion $\tau$. Assuming the tissue to be incompressible:

$$
r^{2}=\frac{r_{0}^{2}}{\lambda_{z}}-\left(\frac{r_{i, 0}^{2}}{\lambda_{z}}-r_{i}^{2}\right)
$$

with $r_{i}$ and $r_{i, 0}$ the inner radius of the cylinder in the deformed and the reference configuration, respectively. The ejection fraction relates $r_{i}$ and $r_{i, 0}$ as follows:

$$
\frac{V_{e j}}{V_{c a v, 0}}=\frac{V_{c a v, 0}-V_{c a v}}{V_{c a v, 0}}=1-\frac{r_{i}^{2}}{r_{i, 0}^{2}} \lambda_{z}
$$

with $V_{c a v, 0}$ and $V_{c a v}$ the cavity volume enclosed by the cylinder in the reference situation and the deformed situation, respectively. Rewriting Eq A3.2 gives:

$$
\left(\frac{r_{i, 0}^{2}}{\lambda_{z}}-r_{i}^{2}\right)=\frac{r_{i, 0}^{2}}{\lambda_{z}} \frac{V_{e j}}{V_{c a v, 0}}
$$

Combination of Eq A3.1 and Eq A3.3 results in:

$$
\begin{aligned}
r & =\sqrt{\frac{r_{0}^{2}}{\lambda_{z}}-\frac{r_{i, 0}^{2}}{\lambda_{z}} \frac{V_{e j}}{V_{c a v}, 0}} \\
& =f\left(r_{0}, r_{i, 0}, V_{c a v, 0}, V_{e j}, \lambda_{z}\right)
\end{aligned}
$$

From continuum mechanics, the deformation field is described by the deformation gradient tensor $\boldsymbol{F}$. Tensor components with respect to the cylindrical coordinate base are stored in matrix $F$, which for the used deformation field is given by:

$$
\underline{F}=\left[\begin{array}{ccc}
\lambda_{r} & 0 & 0 \\
\lambda_{\phi r} & \lambda_{\phi} & \lambda_{\phi z} \\
0 & 0 & \lambda_{z}
\end{array}\right]
$$


with

$$
\begin{aligned}
\lambda_{r} & =\frac{\partial r}{\partial r_{0}}=\frac{r_{0}}{\lambda_{z} r} \quad ; \quad \lambda_{\phi}=\frac{r}{r_{0}} \frac{\partial \phi}{\partial \phi_{0}}=\frac{r}{r_{0}} \\
\lambda_{\phi r} & =r \frac{\partial \phi}{\partial r_{0}}=\frac{\tau z_{0} r_{0}}{r^{2}} ; \quad \lambda_{\phi z}=r \frac{\partial \phi}{\partial z_{0}}=-\tau \lambda_{z}
\end{aligned}
$$

According to Eq 3.3 for the components of reference myofiber direction $\vec{e}_{f, 0}$ :

$$
{\underset{\sim}{f, 0}}_{f}=\left[\begin{array}{lll}
0 & \cos \left(\alpha_{0}\right) & \sin \left(\alpha_{0}\right.
\end{array}\right]^{T}
$$

with $\alpha_{0}$ the myofiber angle defined in the reference configuration. The deformed myofiber direction $\underset{\sim}{\underset{f}{f}}$ is given by:

$$
\underline{\sim}_{f}=\underline{F}_{\sim} \underline{f}_{0} / \lambda_{f}
$$

For the myofiber stretch ratio $\lambda_{f}$ :

$$
\lambda_{f}=\sqrt{e_{f, 0}^{T}\left(\underline{F}^{T} \underline{F}\right) e_{f, 0}}
$$

Combination of Eq A3.5, Eq A3.8 and Eq A3.10 results in:

$$
\lambda_{f}=\sqrt{\left(\cos \left(\alpha_{0}\right) \lambda_{\phi}+\sin \left(\alpha_{0}\right) \lambda_{\phi z}\right)^{2}+\sin ^{2}\left(\alpha_{0}\right) \lambda_{z}^{2}}
$$

Furthermore, the myofiber angle $\alpha$ in the deformed configuration is given by:

$$
\alpha=\arctan \left(\frac{\sin \left(\alpha_{0}\right) \lambda_{z}}{\cos \left(\alpha_{0}\right) \lambda_{\phi}+\sin \left(\alpha_{0}\right) \lambda_{\phi z}}\right)
$$

\section{ACKNOWLEDGEMENTS}

This work was supported by The Netherlands Heart Foundation Grant 2000T036 and University Hospital Maastricht Grant PF155. T. Delhaas is a Clinical Fellow of The Netherlands Heart Foundation (Dr. E. Dekker Fund).

\section{REFERENCES}

1. Arts T, Prinzen FW, Snoeckx LH, Rijcken JM, Reneman RS (1994) Adaptation of cardiac structure by mechanical feedback in the environment of the cell: a model study. Biophys J 66:953961.

2. Arts T, Reneman RS (1988) The importance of geometry of the heart. In: Ter Keurs HE, Noble MIM (Eds) Starling's law of the heart revisited, Kluwer Adacemic Publishers, chapter 8 (ISBN13: $978-0-898-38382-9)$. 
3. Azhari H, Weiss JL, Rogers WJ, Siu CO, Zerhouni EA, Shapiro EP (1993) Noninvasive quantification of principal strains in normal canine hearts using tagged MRI images in 3-D. Am J Physiol Heart Circ Physiol 264:H205-H216.

4. Bishopric NH, Sato B, Webster KA (1992) Beta-adrenergic regulation of a myocardial actin gene via a cyclic AMP-independent pathway. J Biol Chem 267:20932-20936.

5. Bovendeerd PH, Arts T, Huyghe JM, van Campen DH, Reneman RS (1992) Dependence of local left ventricular wall mechancis on myocardial myofiber orientation: a model study. J Biomech 25:1129-1135.

6. Delhaas T, Kroon W, Decaluwe W, Rubbens M, Bovendeerd P, Arts T (2008) Structure and torsion of the normal and the situs inversus totalis cardiac left ventricle. I. Experimental data in humans. Am J Physiol Heart Circ Physiol 295:H197-H201.

7. Donker DW, Volders PG, Arts T, Bekkers BC, Hofstra L, Spätjens RL, Beekman JD, Borgers M, Crijns HJ, Vos MA (2005) End-diastolic myofiber stress and ejection strain increase with ventricular volume overload: Serial in-vivo analyses in dogs with complete atrioventricular block. Basic Res Cardiol 100:372-382.

8. Emery JL, Omens JH, McCulloch AD (1997) Strain softening in rat left ventricular myocardium. J Biomech Eng 119:6-12.

9. Geerts L, Bovendeerd P, Nicolay K, Arts T (2002) Characterization of the normal cardiac myofiber field in goat measured with MR-diffusion tensor imaging. Am J Physiol Heart Circ Physiol 283:H139-H145.

10. Guccione JM, McColloch AD, Waldman LK (1991) Passive material properties of intact ventricular myocardium determined from a cylindrical model. J Biomech Eng 113:42-53.

11. Holmes JW (2004) Candidate mechanical stimuli for hypertrophy during volume overload. J Appl Physiol 97:1453-1460.

12. Humphrey JD (2001) Stress, strain and mechanotransduction in cells. J Biomech Eng 123:638641.

13. Hunter PJ, McCulloch AD, ter Keurs HE (1998) Modeling the mechanical properties of cardiac muscle. Prog Biophys Mol Biol 69:289-331.

14. MacGowan GA, Shapiro EP, Azhari H, Siu CO, Hees PS, Hutchins GM, Weiss JL, Rademakers FE (1997) Noninvasive measurement of shortening in the fiber and cross-fiber directions in the normal human left ventricle and in idiopathic dilated cardiomyopathy. Circ 96:535-541.

15. Matsumura H, Aizawa $Y$, Kumaki K (1990) Myocardial architecture in situs inversus viscerum totalis. In: Clark EB, Takao A (Eds) Developmental cardiology: morphogenesis and function, Futura Pub Co, Mount Kisco, pp. 605-624 (ISBN-13: 978-0-879-93382-1).

16. Nielsen PM, LeGrice IJ, Smaill BH, Hunter PJ (1991) Mathematical model of geometry and fibrous structure of the heart. Am J Physiol Heart Circ Physiol 260:H1365-H1378.

17. Rijcken J, Bovendeerd PH, Schoofs AJ, van Campen DH, Arts T (1999) Optimization of cardiac fiber orientation for homogeneous fiber strain during ejection. Ann Biomed Eng 27:289-297.

18. Rodriguez EK, Hoger A, McCulloch AD (1994) Stress-dependent finite growth in soft elastic tissues. J Biomech 27:455-467.

19. Schlüter KD, Piper HM (1999) Regulation of growth of adult cardiomyocytes. FASEB J 13:S17S22.

20. Scollan DF, Holmes A, Winslow R, Forder J (1998) Histological validation of myocardial microstructure obtained from diffusion tensor magnetic resonance imaging. Am J Physiol Heart Circ Physiol 275:H2308-H2318.

21. Streeter DD Jr, Spotnitz HM, Platel DJ, Ross J, Sonnenblick EH (1969) Fiber orientation in the canine left ventricle during diastole and systole. Circ Res 24:339-347.

22. Taber LA (1995) Biomechanics of growth, remodeling, and morphogenesis. Appl Mechan Rev 48:487-545.

23. Taber LA, Perucchio R (2000) Modeling heart development. J Elas 61:165-197. 


\section{Chapter 3}

24. Ubbink SW, Bovendeerd PH, Delhaas T, Arts T, van de Vosse FN (2006) Towards model based analysis of cardiac MR tagging data: relation between left ventricular shear strain and myofiber orientation. Med Imag Anal 10:632-641.

25. van der Vusse GJ, Arts T, Glatz JF, Reneman RS (1990) Transmural difference in energy metabolism of the left ventricle: fact or fiction. J Mol Cell Cardiol 22:23-37.

26. Vendelin M, Bovendeerd PH, Engelbrecht J, Arts T (2002) Optimizing ventricular fibers: uniform strain or stress, but not atp consumption, leads to high efficiency. Am J Physiol Heart Circ Physiol 283:H1072-H1081.

27. Waldman LK, Nosan D, Villarreal F, Covell JW (1988) Relation between transmural deformation and local myofiber direction in canine left ventricle. Circ Res 63:550-562. 




\section{Computational analysis of the myocardial structure Adaptation of myofiber orientations through deformation in three dimensions}

Wilco Kroon ${ }^{2}$, Tammo Delhaas ${ }^{1,2}$, Peter Bovendeerd ${ }^{4}$, and Theo Arts $^{3}$

Department of ${ }^{1}$ Pediatrics, ${ }^{2}$ Physiology and ${ }^{3}$ Biophysics, Cardiovascular Research Institute Maastricht, University Hospital Maastricht, The Netherlands; and ${ }^{4}$ Department of Biomedical Technology, Eindhoven University of Technology, Eindhoven, The Netherlands.

Med Imag Anal. Doi:10.1016/j.media.2008.06.015, 2008 


\section{ABSTRACT}

Deformation and structure of the cardiac wall can be assessed non-invasively by imaging techniques such as magnetic resonance imaging. Understanding the (patho-)physiology that underlies the observed deformation and structure is critical for clinical diagnosis. However, much about the genesis of deformation and structure is unknown. In the present computational model study, we hypothesize that myofibers locally adapt their orientation to achieve minimal fiber cross-fiber shear strain during the cardiac cycle. This hypothesis was tested in a 3D finite element model of left ventricular (LV) mechanics by computation of tissue deformations and subsequent adaptation of initial myofiber orientations towards those in the deformed tissue. As a consequence of adaptation, local myofiber peak stress, strain during ejection and stroke work density were all found to increase by at least $10 \%$, as well as to become $50 \%$ more homogeneous throughout the wall. Global LV work (peak systolic pressure, stroke volume and stroke work) increased significantly as well (>9\%). The model-predicted myofiber orientations were found to be similar to those in experiments. To the best of our knowledge the presented model is the first that is able to simultaneously predict a realistic myocardial structure as well as to account for the experimentally observed homogeneity in local myofiber strain during ejection.

\subsection{INTRODUCTION}

Deformation and structure of the cardiac wall can be assessed by magnetic resonance imaging techniques such as tagging (3) and diffusion tensor imaging (MRDTI) $(13,16)$, respectively. Understanding the (patho-)physiology that underlies the observed deformation and structure is critical for clinical diagnosis. However, much about the genesis of deformation and structure is unknown. In this study, we propose to use a computational model to test hypothesized processes involved in the genesis of the myocardial structure. A correct computational estimate of myocardial structure as obtained with MRDTI would substantiate a possible role for the hypothesized processes in real physiology.

An important observation is that the cardiac left ventricle (LV) adapts its cavity and wall volume in response to altered mechanical loading $(10,17,23)$. Furthermore, models of cardiac mechanics have demonstrated that the load distribution in the wall is highly dependent on the myofiber orientations, i.e., variation of the myofiber orientations within the range of experimental data resulted in significant differences in myofiber stresses and strains $(7,28)$. Experimental data, however, show that myofiber shortening during ejection exhibits little heterogeneity throughout the wall (21). Heterogeneity in metabolism and oxygen consumption was found to be limited as well, which suggests that myocardial work is 
homogeneously distributed (29). These observations suggest the existence of an adaptive process controlling myofiber orientation so that mechanical load is homogeneously distributed over the wall $(2,13,26)$.

Rijcken et al. 1999 hypothesized that myofiber orientations are such that myofiber shortening throughout the cardiac wall is distributed uniformly. In their study, a polynomial description of the spatial distribution of myofiber orientations was used. Subsequently, coefficients of the polynomial were optimized for minimal heterogeneity in myofiber shortening during ejection. At the minimum, the myofiber angles were found to be realistic, thus supporting the idea that myofiber directions are such that loading is homogeneously distributed. Translation of the model to physiology implies that heterogeneity can be sensed and that myofiber orientations can be adapted at a spatial scale larger than that of the individual myofiber. This is unlikely to be realistic. Instead, it is more likely that cells adjust their orientation locally in response to a local stimulus (2).

passive tissue

myofiber
(3) adaptation direction
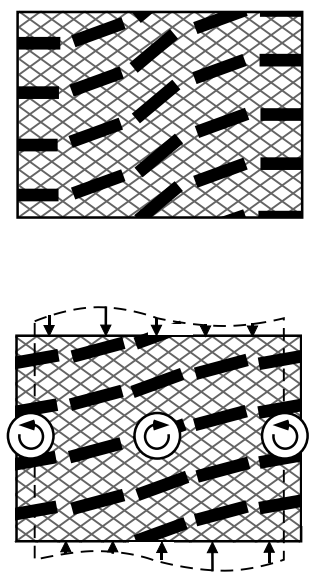

adapted passive tissue deformed tissue

(A)

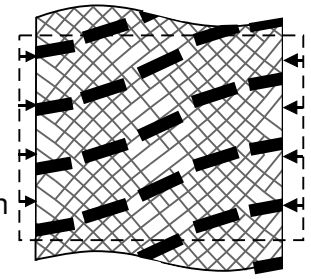

turnover contraction

(C)

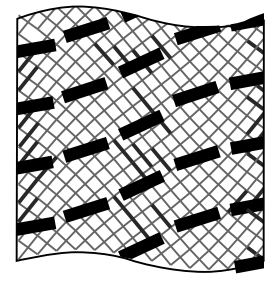

(B)

myofiber

relaxation

adapted deformed tissue

Figure 4.1. Schematic description of the hypothesis on adaptation of myofiber orientation. (A) Due to myofiber contraction the tissue exhibits fiber cross-fiber shear deformation. As a result, the extra-cellular matrix is mechanically loaded which leads to a local loss of integrity. (B) New connections in the deformed tissue restore the integrity, supporting the deformed myofiber orientations. (C) Upon relaxation the myofibers do not fully return to their original orientation and adaptation of the myofiber orientation towards the deformed orientation has taken place. The process will continue until a situation is reached where fiber cross-fiber shear deformation is minimal. 
For collagenous tissues, models have been proposed for adaptation of local collagen fiber orientation in response to local tissue deformation $(5,11,12,33)$. Application to passively loaded tissues such as the aortic valve leaflet, the artery or articular cartilage, showed that alignment of collagen in between principal deformation directions results in a structure similar to that in the real tissue. However, in the actively contracting cardiac wall, principal directions of deformation do not correlate directly to the myofiber orientation $(4,21,24,30)$. Consequently, an alternative hypothesis is needed for the myocardial tissue.

In this study, we build upon the hypothesis that the local myocardial structure is determined by local deformation and assume that myofibers locally adapt their orientation to achieve minimal shear strain during the cardiac cycle. It is known that the extra-cellular matrix (ECM) acts as an important regulator of the myocardial structure. For instance, a disease that affects the ECM integrity such as osteogenesis imperfecta was found to coincide with abnormal myofiber orientations (32). We assume that fiber cross-fiber shear deformation, as induced by systolic myofiber contraction, reflects mechanical load of the connections between the ECM and the myofibers. These forces locally affect the structural integrity of the myocardium (Fig 4.1). In the process of continuous ECM turnover, rearrangement of connections would lead to reduction of forces between the myofibers and the ECM. Eventually, a structure would appear with minimal fiber cross-fiber shear.

The above hypothesized mechanism was investigated in a finite element model of LV mechanics (18), extended with a closed-loop model of the circulation. The model-predicted structure was quantitatively compared with MRDTI data from literature. In addition, we analyzed the effect of adaptation of the myofiber orientations on local mechanics and global hemodynamics.

\subsection{METHODS}

\subsubsection{Model of adaptation of the myofiber orientations}

During systole, fiber cross-fiber shear strain as induced by myofiber contraction locally generates forces between the extra-cellular matrix (ECM) and the myofibers, resulting in a local loss of myocardial integrity (Fig 4.1). It is assumed that during the turnover of connections between the ECM and the myofibers, new connections are formed so that the unloaded myofiber direction $\vec{e}_{f, 0}$ evolves towards the deformed myofiber direction $\vec{e}_{f}$. This evolution is phenomenologically described by:

$$
\frac{\partial \vec{e}_{f, 0}}{\partial t}=\frac{1}{\kappa}\left(\vec{e}_{f}(\boldsymbol{F})-\vec{e}_{f, 0}\right)
$$


with $\kappa$ being a time constant. The tissue deformation with respect to the unloaded tissue configuration is quantified by the deformation gradient tensor:

$$
\boldsymbol{F}=\left(\vec{\nabla}_{0} \vec{u}\right)^{T}+\boldsymbol{I}
$$

with $\vec{u}$ the local tissue displacement vector, $\boldsymbol{I}$ the unity tensor, and $\vec{\nabla}_{0}$ the spatial gradient operator with respect to the unloaded tissue configuration.

Because rigid body rotations are irrelevant to adaptation, they should be excluded from relation Eq 4.1. Rigid body rotations $\boldsymbol{R}$ are removed from $\boldsymbol{F}$ by polar decomposition (22):

$$
\boldsymbol{U}=\boldsymbol{R}^{-1} \cdot \boldsymbol{F}=\sqrt{\boldsymbol{C}} ; \quad \boldsymbol{C}=\boldsymbol{F}^{T} \cdot \boldsymbol{F}
$$

with $C$ the Cauchy strain tensor. The tensor $U$ describes the deformation excluding rigid body rotations, but including shear deformation. The deformed fiber direction $\vec{e}_{f}$ to be used in Eq 4.1 is then given by ${ }^{\dagger}$ :

$$
\vec{e}_{f}=\frac{U \cdot \vec{e}_{f, 0}}{\lambda_{f}} ; \quad \lambda_{f}=\left|\boldsymbol{U} \cdot \vec{e}_{f, 0}\right|
$$

with $\lambda_{f}$ the myofiber stretch ratio. Note that by excluding rigid body rotations from the deformation, the adaptation of the unloaded myofiber direction according to Eq 4.1 becomes solely dependent on fiber cross-fiber shear strain.

\subsubsection{Model of cardiac mechanics}

Tissue displacements $\vec{u}$ during the cardiac cycle are computed with the finite element (FE) model of left ventricular (LV) mechanics as previously presented by Kerckhoffs et al. 2003. The model will be summarized briefly.

Material properties The cardiac tissue is modeled as a nearly incompressible, nonlinearly elastic, transversely isotropic, fiber-reinforced material. Tissue Cauchy stress $\sigma$ is the sum of the passive tissue matrix stress $\sigma_{m}$ and the active myofiber stress $\sigma_{a}$, uniaxially generated in the myofiber direction $\vec{e}_{f}$ :

$$
\sigma=\sigma_{m}+\sigma_{a} \vec{e}_{f} \vec{e}_{f}
$$

The relation between deformation and the passive tissue stress is described by a strain energy density function $W$ :

$$
\boldsymbol{\sigma}_{m}=\tilde{\boldsymbol{\sigma}}_{m}\left(W\left(\boldsymbol{C}, \vec{e}_{f}, \boldsymbol{F}\right)\right)
$$

${ }^{\dagger}$ Footnote: In terms of principal deformation directions $\boldsymbol{U}=\lambda_{1} \vec{e}_{1} \vec{e}_{1}+\lambda_{2} \vec{e}_{2} \vec{e}_{2}+\lambda_{3} \vec{e}_{3} \vec{e}_{3}$ (22), where $\vec{e}_{i}$ and $\lambda_{i}$ are the principal deformation directions and magnitudes with respect to the unloaded reference configuration (eigenvectors and eigenvalues of $\boldsymbol{U}$ ), respectively. In case one of the principal deformation directions coincides with the fiber direction, e.g., $\vec{e}_{1}=\vec{e}_{f, 0}$, then $\vec{e}_{f}=\lambda_{1} \vec{e}_{1} \vec{e}_{1} \cdot \vec{e}_{f, 0} / \lambda_{f}=$ $\vec{e}_{f, 0}$ and the fiber direction stays the same. Hence, adaptation of the unloaded myofiber orientation occurs only in case no principal deformation direction coincides with the unloaded myofiber direction, i.e., in case of fiber cross-fiber shear strain. 
Active myofiber stress generated during contraction is a function of sarcomere length $l_{s}$, sarcomere shorting velocity $\partial l_{s} / \partial t$ and the time since mechanical activation $t_{a}$ :

$$
\sigma_{a}=\tilde{\sigma}_{a}\left(l_{s}, \frac{\partial l_{s}}{\partial t}, t_{a}\right)
$$

Initial geometry, myofiber orientation, and sarcomere length The unloaded LV is represented by a truncated ellipsoid with a cavity and wall volume of 44 and $120 \mathrm{ml}$, respectively. Both are representative volumes for the dog LV (7). During adaptation the unloaded LV geometry remains unaltered. Myofiber orientation $\vec{e}_{f, 0}$ in the initial unloaded LV is prescribed by helix angle $\alpha_{h, 0}$ and transverse angle $\alpha_{t, 0}$ as depicted in Fig 4.2A. The initial values for $\alpha_{h, 0}$ are obtained from Bovendeerd et al. 1998 while values for $\alpha_{t, 0}$ are set to zero. Note that the unloaded myofiber orientation is directly adapted according to Eq 4.1, i.e., no additional constraints are imposed on the individual myofiber angles. The sarcomere length in the initial unloaded tissue configuration is uniformly set to $1.9 \mu \mathrm{m}$.

Balance laws The balance of linear momentum renders the displacements. Neglecting body forces and inertial forces, the balance of linear momentum is given by:

$$
\vec{\nabla} \cdot \boldsymbol{\sigma}=\overrightarrow{0}
$$

The tissue is externally loaded by a cavity pressure $p_{l v}$ normal to the endocardial surface $\Gamma_{e n}$, while the epicardial surface $\Gamma_{e p}$ is assumed to be traction-free:

$$
\begin{aligned}
& \sigma \cdot \vec{n}=-p_{l v} \vec{n} \text { on } \Gamma_{e n} \\
& \sigma \cdot \vec{n}=\overrightarrow{0} \quad \text { on } \Gamma_{e p}
\end{aligned}
$$

where $\vec{n}$ denotes the direction of the unit outer normal at the surface.

\begin{tabular}{|llll} 
Parameter & Description & Value & Unit \\
& Venous resistance & $4.5 \times 10^{6}$ & $\mathrm{~Pa} \cdot \mathrm{s} \cdot \mathrm{m}^{-3}$ \\
$R_{\text {ven }}$ & Arterial resistance & $1.5 \times 10^{7}$ & $\mathrm{~Pa} \cdot \mathrm{s} \cdot \mathrm{m}^{-3}$ \\
$R_{\text {per }}$ & Peripheral resistance & $1.4 \times 10^{8}$ & $\mathrm{~Pa} \cdot \mathrm{s} \cdot \mathrm{m}^{-3}$ \\
$C_{\text {ven }}$ & Venous compliance & $3.0 \times 10^{-8}$ & $\mathrm{~m}^{3} \cdot \mathrm{Pa}^{-1}$ \\
$C_{\text {art }}$ & Arterial resistance & $1.0 \times 10^{-9}$ & $\mathrm{~m}^{3} \cdot \mathrm{Pa}^{-1}$ \\
$V_{\text {ven }, 0}$ & Venous blood volume at zero pressure & $3.2 \times 10^{-3}$ & $\mathrm{~m}^{3}$ \\
$V_{\text {art }, 0}$ & Arterial blood volume at zero pressure & $0.5 \times 10^{-3}$ & $\mathrm{~m}^{3}$ \\
$V_{\text {blood }}$ & Total blood volume & $4.4 \times 10^{-3}$ & $\mathrm{~m}^{3}$ \\
\hline & & &
\end{tabular}

Table 4.1. Parameter values for the lumped parameter model of the circulation. 
Model of the circulation As an extension to the original model by Kerckhoffs et al. 2003, the finite element model of the LV is incorporated in a lumped parameter (LP) model of the closed systemic circulation to supply hemodynamic boundary conditions. The systemic circulation is modeled with a series of constant resistances $R$ and capacitances $C$ (Fig 4.2B). A valve is modeled as an ideal diode. Pressure drop $\Delta p$ across a resistance or a capacitance is given by (6):

$$
\begin{aligned}
\Delta p_{C} & =\frac{V-V_{0}}{C} \\
\Delta p_{R} & =q R
\end{aligned}
$$

with $V$ the volume in the capacitance and $q$ the flow through the resistance. Volume $V_{0}$ represents the volume at zero pressure. Parameter values in the circulation model are listed in Table 4.1. Arterial, venous, and LV cavity volume sum up to a constant total blood volume of $4.4 \times 10^{-3} \mathrm{~m}^{3}$.

Numerical implementation To determine the cardiac tissue displacements from the balance of linear momentum in Eq 4.8 we employ a Galerkin type finite element method, as implemented in the commercial package SEPRAN (SEPRA, Leidschendam, The Netherlands). A modified Newton-Raphson iteration method is used to solve the non-linear equations of conservation of linear momentum.

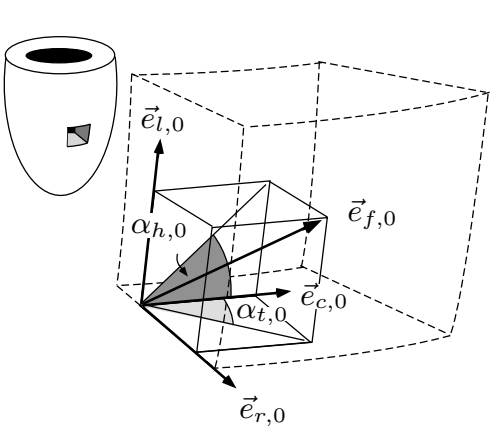

A

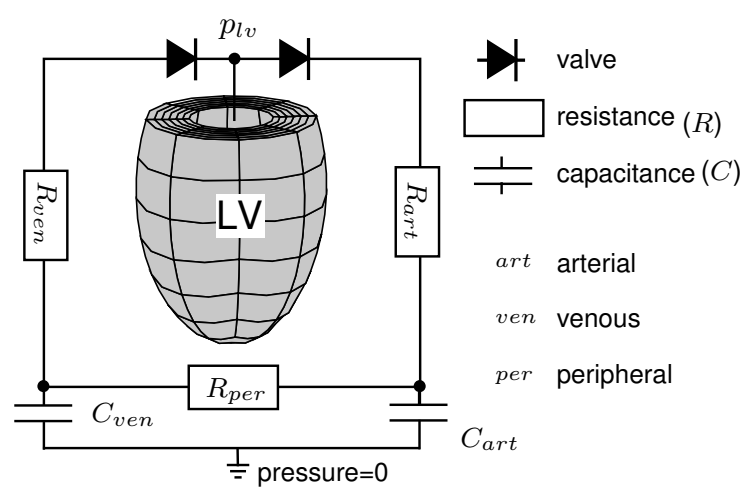

B

Figure 4.2. Computational model of cardiac left ventricular (LV) mechanics. A The unstressed LV is represented by a truncated ellipsoid. Myofiber direction $\vec{e}_{f, 0}$ is quantified by helix angle $\alpha_{h, 0}$ and transverse angle $\alpha_{t, 0}$, with respect to local longitudinal, circumferential, and radial directions $\vec{e}_{l, 0}, \vec{e}_{c, 0}$, and $\vec{e}_{r, 0}$, respectively. B The finite element mesh consists of 312, 27-noded triquadratic hexahedral elements with 3 degrees of freedom (displacements) per node. Circulatory boundary conditions on cavity pressure $p_{l v}$ are modeled by a lumped parameter model. 


\section{Chapter 4}

Spatial discretization occurred with 312, 27-noded, triquadratic hexahedral elements with 3 degrees of freedom (displacements) per node (see Fig 4.2B). Myofiber orientations were defined at each node and interpolated using the same triquadratic elements as used for displacement interpolation. Spatial numerical integration is conducted with a Newton-Cotes scheme. Temporal discretization during the cardiac cycle occurs at a resolution of $\Delta t=1 \mathrm{~ms}$, using an explicit Euler scheme. Coupling between the models of the ventricle and circulation occurs by adjusting the pressure $p_{l v}$ in such a way that the volume change as computed with the FE model equals the volume change as computed with the LP model (19). Adaptation was implemented by altering the unloaded myofiber directions in each nodal point according to Eq 4.1, using the nodal point value of $U$.

\subsubsection{Simulations performed}

The first 10 consecutive cardiac cycles, in which adaptation of the myofiber orientation was not allowed, were used to reach a hemodynamic steady state. Adaptation was simulated over the next 30 cardiac cycles. Contraction of the myofibers was synchronously initiated with a cycle time of $600 \mathrm{~ms}$. Adaptation time constant $\kappa$ was set to $1 \times 10^{4} \mathrm{~ms}$, indicating that, in each simulation time step $\Delta t$, the reference myofiber orientation is changed with $\Delta t / \kappa=1 \times 10^{4}$ times the difference between the reference and the deformed myofiber orientation. Because of the coarseness of the finite element mesh near the apex, we excluded the apex from adaptation (Fig 4.3A).

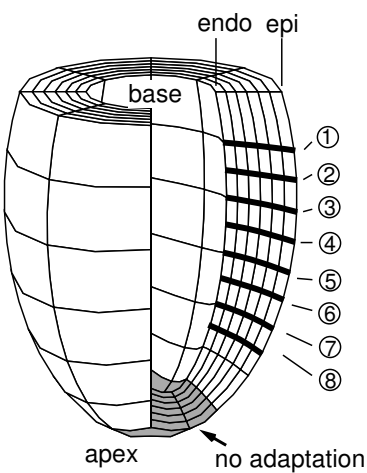

A

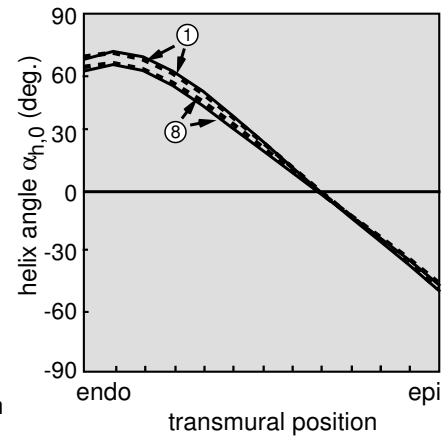

B

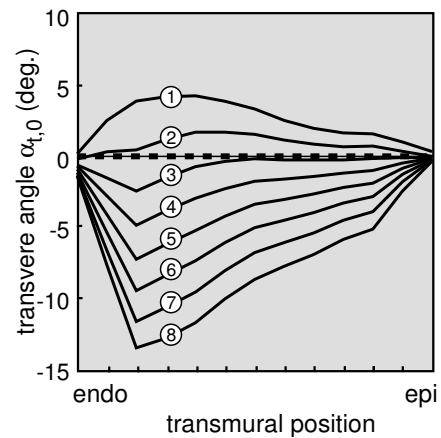

C

Figure 4.3. Transmural course in myofiber angles before (- -) and after (-) myofiber adaptation. A Analysis is done at 8 different longitudinal levels in the LV wall. Near the apex adaptation was not allowed. B Helix angles $\alpha_{h, 0}$. C Transverse angles $\alpha_{t, 0}$. Initially the transverse angle was zero at all levels. 


\subsection{RESULTS}

To asses the effect of adaptation of the myofiber orientation on the structure and function of the LV, we analyzed global hemodynamics and local mechanics in addition to the change in the unloaded myofiber orientations. Baseline values for structure, local mechanics, and global hemodynamics were derived from the hemodynamic steady state at cardiac cycle 10.

\subsubsection{Structural response}

Adaptation of the unloaded myofiber orientations $\vec{e}_{f, 0}$ was quantified by changes in the transmural distribution of myofiber angles $\alpha_{h, 0}$ and $\alpha_{t, 0}$ for 8 levels from apex to base (Fig 4.3A). Angles $\alpha_{h, 0}$ and $\alpha_{t, 0}$ were determined from $\vec{e}_{f, 0}$ in a postprocessing step. Fig 4.3B shows that the adapted helix angles were not significantly different from the initial angles. Apparently, the initial helix angles as determined by Bovendeerd et al. 1998 were already near the steady state values for local adaptation. In contrast, the transverse angles show a significant change, becoming positive near the base and negative near the apex (Fig 4.3C). The absolute maximum of the transverse angle is located near the subendocardium.

\subsubsection{Mechanical load response}

Fig 4.4B shows the local myofiber stress-strain loops at several positions in the wall. The initial structure resulted in an inhomogeneous distribution of myofiber stresses and strains. Peak-systolic stress increased towards the epicardium, while myofiber shortening during ejection was maximum near the endocardium. After adaptation, distribution of myofiber stresses and strains became more homogeneous.

For the grey area indicated in Fig 4.4A, the effect of adaptation on local tissue mechanics is quantified by the change in mean and standard deviation of the maximum myofiber stress, natural myofiber strain during the ejection phase, and stroke work density $w_{f}$. The myofiber stroke work density $w_{f}$ at each point was given by the area enclosed by the myofiber stress-natural strain loop:

$$
w_{f}=\oint \sigma_{f} d \varepsilon_{f} ; \quad \varepsilon_{f}=\ln \left(\lambda_{f}\right)
$$

where $\sigma_{f}$ and $\varepsilon_{f}$ denote the myofiber strain and natural myofiber strain, respectively.

Fig 4.4C shows the evolution of local myofiber mechanics. As a consequence of adaptation, the mean of all parameters increases, while the standard deviation decreases. The latter indicates homogenization of mechanics throughout the wall. 


\subsubsection{Hemodynamics response}

Global cardiac function was assessed through global pump work $W$ as computed from the LV pressure-volume loop by:

$$
W=\oint p_{l v} d V_{l v}
$$

Maximum pressure, stroke volume, and pump work before and after the reorientation were compared. Fig 4.5B shows that as a consequence of adaptation of the myofiber orientation, maximum pressure, stroke volume, as well as pump work increase.

\section{○ valve opening/closure - - - - before reorientation after reorientation}

A

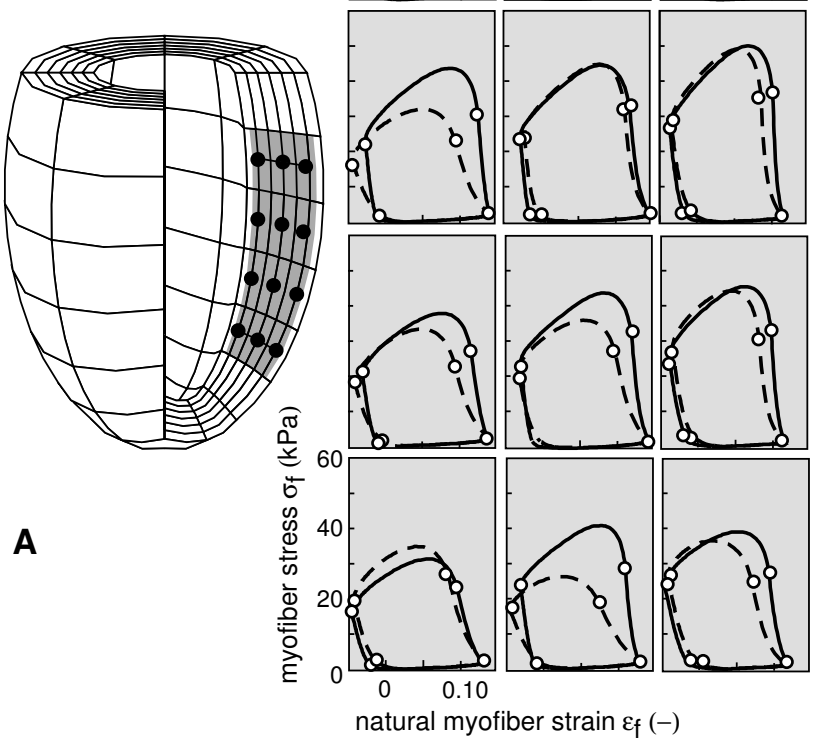

B
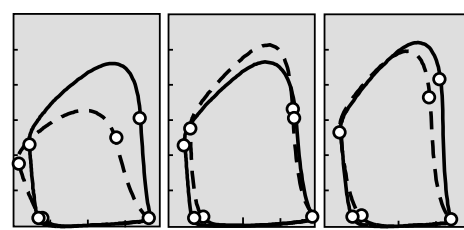

natural myofiber strain $\varepsilon_{f}(-)$

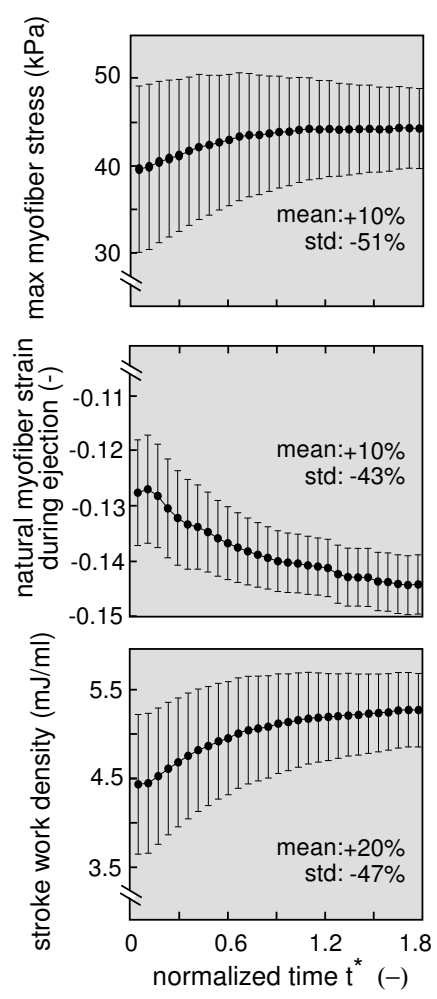

C

Figure 4.4. Change in local mechanics as a consequence of myofiber reorientation. A Analysis includes nodes as indicated by $\bullet$. B Myofiber stress-strain loops before (- -) and after ( $(-)$ adaptation. C Evolution of mean $(\bullet)$ and standard deviation (error bars) of maximum myofiber stress, natural myofiber strain during ejection, and stroke work density as determined for the grey area in $\mathbf{A}$. Time $t$ is normalized with respect to adaptation time constant $\kappa\left(\mathrm{t}^{*}=\mathrm{t} / \kappa\right)$. 


\subsection{DISCUSSION}

The aim of the present study is to investigate the hypothesis that myofibers adapt their orientation to minimize their shear deformation. In a computational model we tested this hypothesis on the ability to reproduce the myofiber orientations as obtained experimentally using MRDTI.

\subsubsection{Comparison between model and experiment}

Fig 4.6 shows the comparison between the model estimated myofiber orientation and experimental data for the free-wall of the LV determined with MRDTI (13). As in the experiment, we express fiber orientation by angles $\alpha_{h, 0}^{*}$ and $\alpha_{t, 0}^{*}$

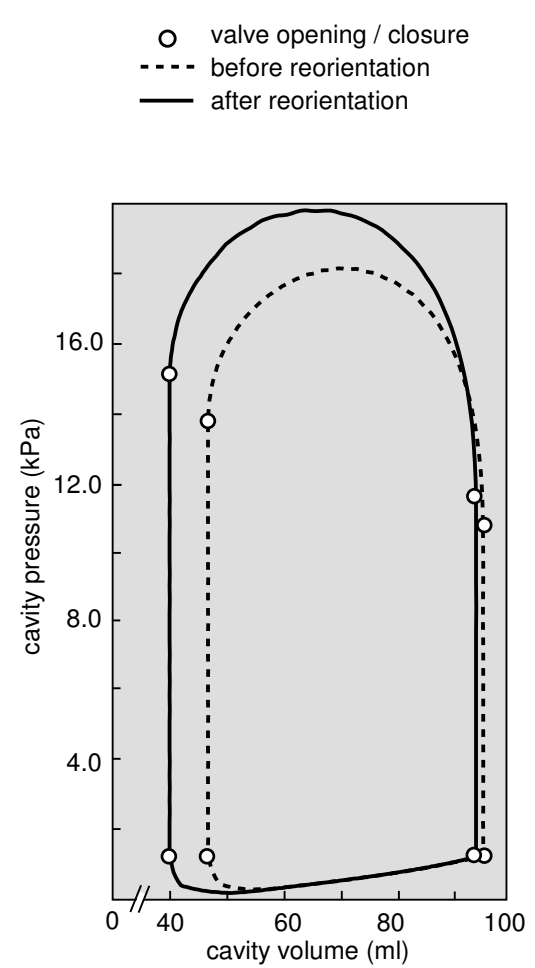

A

Figure 4.5. Hemodynamics during myofiber reorientation. A Pressure-volume loops. B Maximum pressure, stroke volume, as well as stroke work increase as a consequence of adaptation. The percentage indicates the relative change. Time $t$ is normalized with respect to the adaptation time constant $\kappa\left(\mathrm{t}^{*}=\mathrm{t} / \kappa\right)$.

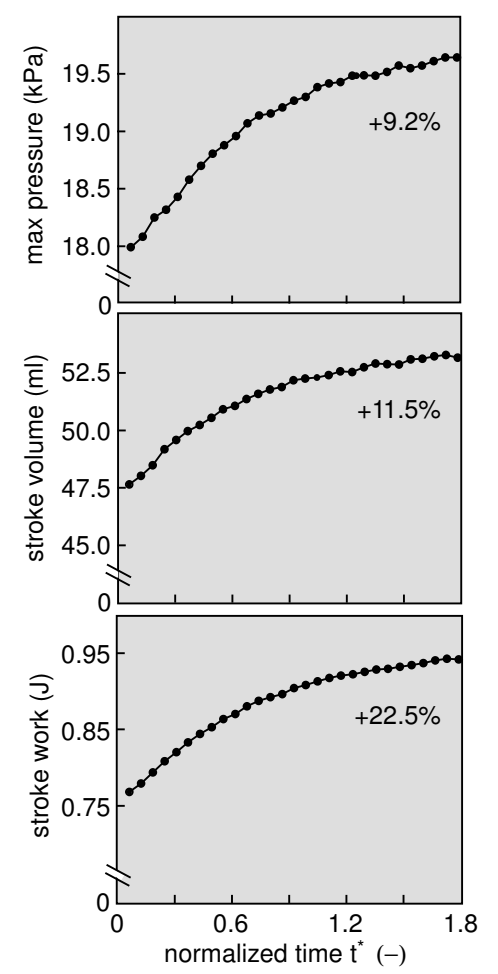

B 
with respect to a local cylindrical basis instead of the ellipsoidal basis as shown in Fig 4.2A. It is noted that this definition yields identical values for $\alpha_{h, 0}^{*}$ and $\alpha_{h, 0}$, $\alpha_{t, 0}^{*}$ and $\alpha_{t, 0}$ in the equatorial plane. As a consequence of adaptation of the orientation, a structure with realistic helix and transverse angles developed out of a non-physiological structure in which the myofiber orientations lacked a transmural component. Predicted helix angles are within experimental range and the experimentally observed change in sign of the transverse angle from positive near the base to negative near the apex is also correctly predicted (Fig 4.6). Furthermore, the longitudinal gradient of the model-predicted transverse angle at midwall is similar to experimental data.

Histological and anatomical findings show that myofibers run parallel to the cardiac surfaces (transverse angle of zero at endocardium and epicardium) and cross-over at the apex and the base (non-zero value of transverse angle in between endocardium and epicardium) (15). Our model predictions are in agreement with these observations. However, to the best of our knowledge no experimental data exist that can confirm whether the model-predicted transmural distribution of the transverse angle as shown in Fig 4.3C is realistic or not. Until now, the signal-to-noise ratio of MRDTI measurements resulted in an accuracy of $6^{\circ}$. Consequently, averaging of a relatively large transmural region was required, yielding only a single transverse angle value per longitudinal level $(13,25)$.

In addition, our simulations show that the hypothesized restructuring of the wall leads to an increased pump function with the same amount of mass (Fig 4.5). Furthermore, heterogeneity of the local mechanics was decreased by almost $50 \%$ (Fig 4.4C). It is noted that both the myocardial structure and reduction in heterogeneity are an outcome of the model, in contrast to the optimization approach of Rijcken 1997, where homogeneity was used as an input criterion. To the best of our knowledge the model presented is the first that is able to predict a realistic myocardial structure, as well as to account for the experimentally observed homogeneity in local mechanics, simultaneously.

\subsubsection{Study assumptions and limitations}

Experimental observations From the large dependence of tissue deformation and tissue stress on the myofiber orientation and the absence of significant heterogeneity in experimentally observed myofiber shortening, it has been deduced that myofiber orientation is controlled by mechanics. However, experimental evidence of load-induced adaptation of myofiber orientations in the adult heart remains elusive (see also Geerts-Ossevoort 2003).

Confocal laser scanning microscopy shows a deviation in cardiac myofiber orientation after a change in tissue loading in the developing (chicken) embryo (27). Furthermore, MRDTI of failing hearts reveals some changes in sheet orientation with respect to normal hearts (16). However, Helm et al. 2006 did not find 
significant changes in the orientation of the individual myofibers. Furthermore, MRDTI data did not show significant changes in infarcted areas (31). In addition, Walker et al. 2005 observed a difference in orientation after surgical repair, but it remains unclear whether this difference can be contributed to adaptation or to a direct effect of the surgical procedure. Possibly, experimental confirmation of load-induced adaptation of myofiber orientations is obscured by the limited accuracy of orientation measurement by MRDTI of $\pm 6^{\circ}$.

Model assumptions At present, there is insufficient information to derive a relation between local deformation and the underlying microstructural changes that occur during adaptation. Therefore, we used a simple first order relation with time constant $\kappa$ to model the adaptation process (Eq 4.1). In the model, $\kappa$ was chosen so that stable convergence of the adaptation occurred in the order of 100 cardiac cycles ( $\sim 10 \mathrm{~s})$. In reality, adaptation of myofiber directions may occur at a much larger timescale. Typically, the half-life of ECM collagen is on the order of days, while the myocytes replace the bulk of their internal proteins within a few weeks (1). However, our goal was not to investigate the kinematics involved in the adaptation, but to test whether our hypothesis could predict a realistic structure.

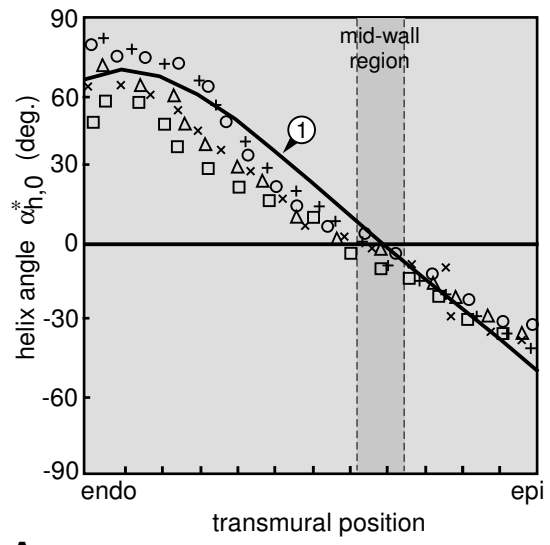

A

Figure 4.6. Comparison of myocardial structure between model and experiment. Modeldetermined angles $\alpha_{h, 0}^{*}$ and $\alpha_{t, 0}^{*}$ are defined with respect to a cylindrical coordinate system, as done in the experiments. A Transmural variation of helix angle $\alpha_{h, 0}^{*}$ near the equator. B Apex-to-base variation in the average of transverse angle $\alpha_{t, 0}^{*}$ near the midwall. The experimental data is adopted from Geerts et al. 2002 and shows the angles in left ventricular free-wall near the base of five unloaded goat hearts as obtained with MRDTI. The encircled numbers indicate apex-to-base levels as shown in Fig 4.3A. The midwall region was defined as the transmural region with an absolute helix angle value of less than $5^{\circ}$. 
Our model only accounts for the adaptation of the myofiber orientations. In reality, adaptation of the cardiac wall mass or adaptation of the systemic vasculature are also likely to occur to tune the demands of the cardiovascular system to the demands of the body on the long term. Although these additional adaptation processes may affect the amplitude of the change in hemodynamics and tissue mechanics, it is expected that the directionality of the relation between adaptation of the structure and the response in hemodynamics and tissue mechanics will remain unaffected.

In the introduction it has been noted that principal directions of deformation do not correlate directly to the myofiber orientation $(4,21,24,30)$. Consequently, we formulated an alternative hypothesis in which myofibers adapt their orientation to minimize shear during the cardiac cycle. However, other hypotheses remain plausible such as alignment of the myofiber to the principal stress direction (5). A future study may be aimed at comparing both hypotheses.

A final note is that our study would benefit from a detailed analysis with different starting conditions and a steady-state solution. However, due to the high computational demand (current simulations took about a week to compute on a standard PC) such an analysis was not yet feasible. Still, different starting conditions are expected to yield small differences with respect to the presented solution. Local adaptation may be considered as a decentralized optimization approach with the local stimulus as local objective function. In general, such an approach does not yield the same solution as would be obtained by centralized optimization where a single objective function is used. Instead, solutions will be found around the same minimum, but a unique solution rarely exists since generally not all local objective functions simultaneously achieve their minimum. Based on experimental data multiple solutions can also be expected. While normal LVs show transmural distributions in helix angle that are similar to what is shown in Fig 4.6A, complete mirroring of the internal organs (Situs Inversus Totalis, SIT) has been shown to result in a transmural distribution that is (partially) mirrored with respect to normal (9). Adaptation models that include myofiber reorientation in a cylindrical LV also indicate the possibility of two solutions to develop from random initial orientations that are each others mirror image (20). Despite the lack of a detailed analysis, however, our model suggests the hypothesized mechanism may effectively (re)structure the heart, while simultaneously homogenizing mechanical loading of the tissue.

Model of cardiac mechanics The model of cardiac mechanics has several limitations as well. Geometry and structure of the LV were assumed rotationally symmetric, while interaction of the LV with the right ventricle (RV) was not taken into account. Although the real geometry of the healthy LV is by good approximation rotationally symmetric, myofiber orientations show significant differences between the free wall and the septum (15). Probably, this asymmetry in structure is due 
to the mechanical interaction between the LV and RV. Since interaction between the LV and RV will have the least effect on the LV free wall, the model predictions were compared with experimental data of the free wall (Fig 4.6). Extending the model with an RV, and subsequent comparison of predicted and measured myofiber orientation would constitute a possible next test for our hypothesis.

\subsection{CONCLUSIONS}

Local adaptation of myofiber orientation to achieve minimal fiber cross-fiber shear during the cardiac cycle was found to lead to a realistic myocardial structure, i.e., myofibers in the model developed a transmural component in their orientation that was similar to those in the real left ventricle as measured with MRDTI. In addition, adaptation was found to lead to significant homogenization of mechanical loading. Furthermore, the adapted structure was able to generate more pump work with the same amount of mass.

\section{REFERENCES}

1. Alberts B, Bray D, Lewis J, Raff M, Roberts K, Watson JD (1994) Molecular Biology of the Cell. Garland publishing, New York.

2. Arts T, Prinzen FW, Snoeckx LH, Rijcken JM, Reneman RS (1994) Adaptation of cardiac structure by mechanical feedback in the environment of the cell: a model study. Biophys $\mathrm{J} 66: 953-$ 961.

3. Axel L, Dougherty L (1989) MR imaging of motion with spatial modulation of magnetization. Am J Physiol Heart Circ Physiol 171:841-845.

4. Azhari H, Weiss JL, Rogers WJ, Siu CO, Zerhouni EA, Shapiro EP (1993) Noninvasive quantification of principal strains in normal canine hearts using tagged MRI images in 3-D. Am J Physiol Heart Circ Physiol 264:H205-H216.

5. Baek S, Rajagopal KR, Humphrey JD (2006) A theoretical model of enlarging intracranial fusiform aneurysms. J Biomech Eng 128:142-149.

6. Bovendeerd PH, Arts T, Delhaas T, Huyghe JM, van Campen DH, Reneman RS (1996) Regional wall mechanics in the ischemic left ventricle: numerical modeling and dog experiments. Am J Physiol Heart Circ Physiol 270:H398-H410.

7. Bovendeerd PH, Arts T, Huyghe JM, van Campen DH, Reneman RS (1992) Dependence of local left ventricular wall mechancis on myocardial myofiber orientation: a model study. J Biomech 25:1129-1135.

8. Bovendeerd PH, Rijcken J, van Campen DH, Schoofs AJG, Nicolay K, Arts T (1998) Optimization of left ventricular muscle fiber orientation. In: Pedersen P, Bendsøe MP (Eds) Proc IUTAM Symposium on Synthesis on Bio Solid Mechanics, Copenhagen, Denmark. pp. 285-295 (ISBN13: 978-0-792-35615-8).

9. Delhaas T, Kroon W, Decaluwe W, Rubbens M, Bovendeerd P, Arts T (2008) Structure and torsion of the normal and the situs inversus totalis cardiac left ventricle. I. Experimental data in humans. Am J Physiol Heart Circ Physiol 295:H197-H201.

10. Donker DW, Volders PG, Arts T, Bekkers BC, Hofstra L, Spätjens RL, Beekman JD, Borgers M, Crijns HJ, Vos MA (2005) End-diastolic myofiber stress and ejection strain increase with 


\section{Chapter 4}

ventricular volume overload: Serial in-vivo analyses in dogs with complete atrioventricular block. Basic Res Cardiol 100:372-382.

11. Driessen NJ, Cox MA, Bouten CV, Baaijens FP (2008) Remodelling of the angular collagen fiber distribution in cardiovascular tissues. Biomech Model Mechanobiol 7(2):93-103.

12. Driessen NJ, Wilson W, Bouten CV, Baaijens FP (2004) A computational model for collagen fibre remodelling in the arterial wall. J Theor Biol 226:53-64.

13. Geerts L, Bovendeerd P, Nicolay K, Arts T (2002) Characterization of the normal cardiac myofiber field in goat measured with MR-diffusion tensor imaging. Am J Physiol Heart Circ Physiol 283:H139-H145.

14. Geerts-Ossevoort L (2003) Cardiac myofiber reorientation: a mechanism for adaptation? Ph.D. thesis Eindhoven University of Technology, Eindhoven, The Netherlands.

15. Gilbert SH, Benson AP, Li P, Holden AV (2007) Regional localisation of left ventricular sheet structure: integration with current models of cardiac fibre, sheet and band structure. Eur $\mathrm{J}$ Cardiothorac Surg 32:231-249.

16. Helm PA, Younes L, Beg MF, Ennis DB, Leclercq C, Faris OP, McVeigh E, Kass D, Miller MI, Winslow R (2006) Evidence of structural remodeling in the dyssynchronous failing heart. Circ Res 98:125-132.

17. Holmes JW (2004) Candidate mechanical stimuli for hypertrophy during volume overload. J Appl Physiol 97:1453-1460.

18. Kerckhoffs RC, Bovendeerd PH, Kotte JC, Prinzen FW, Smits K, Arts T (2003) Homogeneity of cardiac contraction despite physiological asynchrony of depolarization: a model study. Ann Biomed Eng 31:536-547.

19. Kerckhoffs RC, Neal ML, Gu Q, Bassingthwaighte JB, Omens JH, McCulloch AD (2007) Coupling of a 3D finite element model of cardiac ventricular mechanics to lumped systems models of the systemic and pulmonic circulation. Ann Biomed Eng 35:1-18.

20. Kroon W, Delhaas T, Bovendeerd P, Arts T (2008) Structure and torsion in the normal and situs inversus cardiac left ventricle. II. Modeling cardiac adaptation to mechanical loading. Am J Physiol Heart Circ Physiol 295:H202-H210.

21. MacGowan GA, Shapiro EP, Azhari H, Siu CO, Hees PS, Hutchins GM, Weiss JL, Rademakers FE (1997) Noninvasive measurement of shortening in the fiber and cross-fiber directions in the normal human left ventricle and in idiopathic dilated cardiomyopathy. Circ 96:535-541.

22. Malvern LE (1969) Introduction to the Mechanics of a Continuous Medium. Prentice-Hall Inc., Englewood Cliffs, New Jersey.

23. Omens JH (1998) Stress and strain as regulators of myocardial growth. Prog Biophys Mol Biol 69:559-572.

24. Prinzen FW, Arts T, van der Vusse GJ, Reneman RS (1984) Fiber shortening in the inner layers of the left ventricular wall as assessed from epicardial deformation during normoxia and ischeme. J Biomech 17:801-811.

25. Rijcken J (1997) Optimization of left ventricular muscle fiber orientation. Ph.D. thesis Eindhoven University of Technology, Eindhoven, The Netherlands.

26. Rijcken J, Bovendeerd PH, Schoofs AJ, van Campen DH, Arts T (1999) Optimization of cardiac fiber orientation for homogeneous fiber strain during ejection. Ann Biomed Eng 27:289-297.

27. Tobita K, Garrison JB, Liu LJ, Tinney JP, Keller BB (2005) Three-dimensional myofiber architecture of the embryonic left ventricle during normal development and altered mechanical loads. Anat Rec A Discov Mol Cell Evol Biol 283:193-201.

28. Ubbink SW, Bovendeerd PH, Delhaas T, Arts T, van de Vosse FN (2006) Towards model based analysis of cardiac MR tagging data: relation between left ventricular shear strain and myofiber orientation. Med Imag Anal 10:632-641.

29. van der Vusse GJ, Arts T, Glatz JF, Reneman RS (1990) Transmural difference in energy metabolism of the left ventricle: fact or fiction. J Mol Cell Cardiol 22:23-37. 
30. Waldman LK, Nosan D, Villarreal F, Covell JW (1988) Relation between transmural deformation and local myofiber direction in canine left ventricle. Circ Res 63:550-562.

31. Walker JC, Guccione JM, Jiang Y, Zhang P, Wallace AW, Hsu EW, Ratcliffe MB (2005) Helical myofiber orientation after myocardial infarction and left ventricular surgical restoration in sheep. J Thorac Cardiovasc Surg 129:382-390.

32. Weis SM, Emery JL, Becker KD, McBride DJ Jr, Omens JH, McCulloch AD (2000) Myocardial mechanics and collagen structure in the osteogenesis imperfecta murine (oim). Circ Res 87:663-669.

33. Wilson W, Driessen NJ, van Donkelaar CC, Ito K (2006) Prediction of collagen orientation in articular cartilage by a collagen remodeling algorithm. Osteoarthr Cartil 14:1196-1202. 



\section{Adaptation of myofiber orientations through deformation}

Comparison between shear strains in model and experiment 


\begin{abstract}
Mechanical load plays an important role in cardiac growth and remodeling. In a previous study, we hypothesized that fiber cross-fiber shear acts as a stimulus for remodeling of the pattern of myofiber orientations (Kroon et al. 2008). This hypothesis was tested in a mathematical model of cardiac mechanics. We found that, after reorientation in response to fiber cross-fiber shear, model-predicted longitudinal and transmural components of myofiber orientation were within the range of those determined experimentally using magnetic resonance diffusion tensor imaging. In this study, we investigated whether circumferential-radial shear and torsion as determined experimentally with magnetic resonance tagging can be reproduced in the model as well. Our results show that after myofiber reorientation, differences in circumferential-radial shear and torsion between model and experiment were reduced significantly. However, a complete match could not be obtained through shear-induced myofiber reorientation alone.
\end{abstract}

\title{
5.1 INTRODUCTION
}

It is well recognized that cardiac cells such as fibroblasts and myocytes respond to changes in mechanical load. For instance, stretch may significantly alter myocardial protein synthesis and enzymatic activity $(10,12-14)$. As such, mechanical load plays an important role in cardiac growth and remodeling. However, details on stimulus-response relation remain unclear.

In a previous mathematical model study, we investigated the hypothesis that fiber cross-fiber shear deformation acts as a stimulus for reorientation of the myofibers (8). Near the steady-state, model-predicted longitudinal and transmural components of the myofiber orientations were well within the range of those determined experimentally with magnetic resonance diffusion tensor imaging (MRDTI). These results suggest that fiber cross-fiber shear strain plays an important role in adaptation of the myofiber orientations. However, due to inaccuracies in MRDTI measurements, experimental data on myofiber orientations has a relatively broad range (6). Thus, direct comparison of model-predicted orientations with experimental data is not a very critical check for the model.

Cardiac tissue deformation was found to be highly sensitive to the pattern of myofiber orientations. In particular circumferential-radial shear $\left(E_{c r}\right)$ and torsion ( $\tau$, negative of circumferential-axial shear strain) have been found to be sensitive $(2,15)$, suggesting that comparison of shear strains would constitute a (more) critical test. In this study, we compare left ventricular (LV) shear strains as computed with the model before and after myofiber reorientation with those determined from magnetic resonance tagging (MRT) experiments. 


\subsection{METHODS}

\subsubsection{Experimental assessment of LV wall strains with MRT}

Image acquisition In three healthy subjects (age 28-33 years) LV deformation was assessed non-invasively using MRT. Three parallel short-axis slices of the heart were imaged with a distance to the basal plane of one third, one half, and two thirds of the apex-to-base distance; the basal, midventricular, and apical slice, respectively (Fig 5.1A). Tissue was grid-tagged using spatial modulation of magnetization (1), triggered on the R-wave of the electrocardiogram. During the cardiac cycle, the deformed grid was imaged in each slice (tagging images) with a time interval of about $20 \mathrm{~ms}$ (Fig 5.1B). Besides tagging images, cine images were recorded for LV contouring.

Postprocessing The LV wall was outlined in each slice by manually contouring the endo- and epicardial boundaries in the cine images. Subsequently, displacement maps were determined from the tagging images, following Delhaas

A

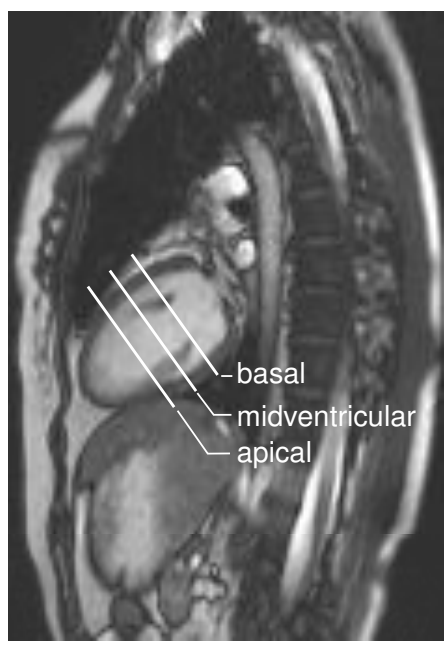

B
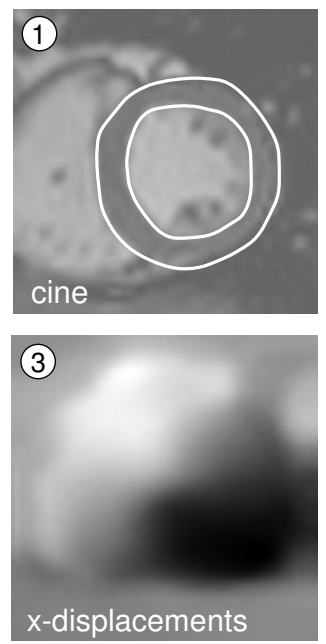
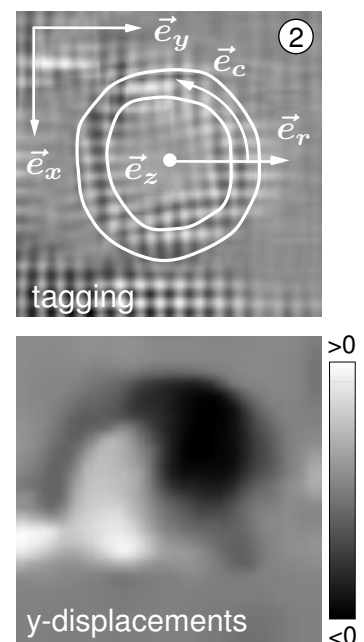

Figure 5.1. A Long-axis MR slice showing the positions of the three short-axis slices, imaged in the cardiac left ventricle (LV). B Short-axis MR images showing the heart as viewed from the apex. (1) LV endo- and epicardial boundaries are contoured in a reference cine image, and (2) subsequently projected on the tagging images. (3) Displacement components along directions $\vec{e}_{x}$ and $\vec{e}_{y}$ are derived through correlation of consecutive tagging images. These displacements are converted into circumferential strain $\left(E_{c c}\right)$, circumferential radial shear strain $\left(E_{c r}\right)$, and torsion $(\tau)$ as expressed with respect to a right-handed coordinate system $\left\{\vec{e}_{r}, \vec{e}_{c}, \vec{e}_{z}\right\}$. Direction $\vec{e}_{z}$ points towards the reader. 
et al. 2008. The deformation gradient tensor was determined by spatial differentiation of the displacement maps. Next, the Green-Lagrange strain tensor was determined referenced with respect to begin-ejection (first image frame), and written in components with respect to a right-handed cylindrical coordinate system $\left\{\vec{e}_{r}, \vec{e}_{c}, \vec{e}_{z}\right\}$. The center of gravity of the pixels in the LV wall was chosen as origin for this coordinate system (Fig 5.1B). $E_{c c}$ and $E_{c r}$ were used for further analysis. For definition of $E_{c r}$, see Fig 5.2A.

Strains were averaged in transmural direction, with weight factors $w\left(\bar{r}_{i}\right)$ defined according to:

$$
w\left(\bar{r}_{i}\right)=\frac{\left(1-\bar{r}_{i}^{2}\right)^{2}}{\sum_{i=1}^{N}\left(1-\bar{r}_{i}^{2}\right)^{2}}
$$

where $\bar{r}_{i}$ is a normalized transmural coordinate, running from -1 at the endocardial contour to +1 at the epicardial contour, and $N$ is the number of pixels in the LV wall. The obtained transmural averages were subsequently averaged in the circumferential direction.

Since no long-axis tagging images were available, circumferential-axial shear strain $\left(E_{c z}\right)$ had to be determined from displacement maps in the short-axis. As an estimate for $E_{c z}$, torsion $\tau$ was calculated in a section between subsequent short-axis slices; $\tau$ was defined as the axial gradient in average wall rotation $\Phi$

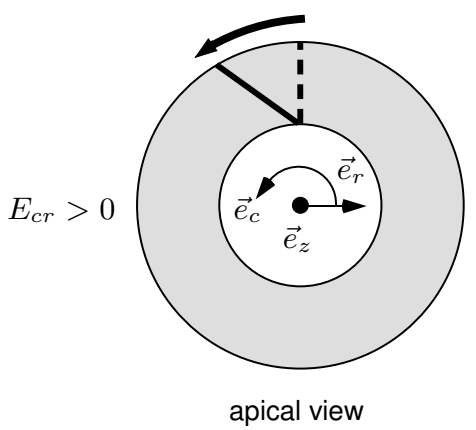

A

Figure 5.2. Circumferential-radial shear strain $E_{c r}$ and torsion $\tau$ are defined with respect to a right-handed cylindrical coordinate system $\left\{\vec{e}_{r}, \vec{e}_{c}, \vec{e}_{z}\right\}$. A $E_{c r}$ quantifies the transmural gradient in rotation. In case the epicardium rotates in circumferential direction $\vec{e}_{c}$ relative to the endocardium, $E_{c r}$ is positive. B $\tau$ quantifies the axial gradient in rotation. In case the base rotates more in circumferential direction $\vec{e}_{c}$ than the apex, $\tau$ is positive. Notice that according to the conventional definition of $\tau$ in Eq 5.2, a positive $\tau$ implies a negative circumferential-axial shear $E_{c z}$. 
multiplied by the average outer radii $r_{o}$ (Fig 5.2B):

$$
\tau=\frac{\Phi^{u}-\Phi^{l}}{d} \frac{r_{o}^{u}+r_{o}^{l}}{2} \quad \text { with } \quad r_{o}=\sqrt{\frac{A_{m w}+\frac{1}{2} A_{w}}{\pi}}
$$

with $d$ the inter-slice distance. Superscripts $u$ and $l$ denote values for the upper and lower slice of the section, respectively. Midwall area $A_{m w}$ was estimated for each frame as the area enclosed by the average radius of the pixels in the wall (midwall radius). Wall area $A_{w}$ was calculated in the reference frame as the area between the epi- and endocardial contours.

To relate timing of the MRT images to phases in the cardiac cycle, the cavity volume as enclosed by the three slices was computed using the cavity areas and the inter-slice distance. Differentiation of this volume with respect to time yielded an estimation of mitral inflow and aortic outflow. Zero crossings of this signal defined moments of transition between phases in the cycle.

\subsubsection{Determining LV wall strains with a mathematical model}

Mathematical model The applied models of cardiac mechanics and myofiber reorientation have been described before $(7,8)$. In short, the stress-free LV geometry is represented by a truncated ellipsoid. The LV wall is composed of myofibers. Their orientation in the stress-free geometry is quantified by the helix angle $\alpha_{h, 0}$ describing the inclination of the myofiber with the circumference, and by the transverse angle $\alpha_{t, 0}$ describing the endo-to-epicardial cross-over of the myofiber. Total wall stress is composed of a passive component, related to deformation, and an active component, related to contraction. The passive stressstrain response of the tissue is non-linear and transversely isotropic with respect to the myofiber orientation. Local active myofiber stress is a function of time after activation, sarcomere length, and sarcomere shortening velocity. Sarcomere length in the stress-free state is constant throughout the wall and equals $1.9 \mu \mathrm{m}$. Mechanical activation of the LV is synchronous with a cycle time of 600 milliseconds. Hemodynamic preload and afterload as imposed on the LV by the vascular system are represented by a simple closed-loop lumped parameter (windkessel) model. Deformation is computed from the balance of linear momentum using the finite element method (FEM).

Locally determined fiber cross-fiber shear was used to adapt the orientation of the myofibers in the stress-free geometry. Initial helix angles were set to values that were determined after global optimization to achieve homogeneous myofiber strain during ejection (3). Initial transverse angles were set to zero. Subsequently, myofiber orientations were locally adapted during 30 cardiac cycles (8). The myofiber angles before and after adaptation are shown in Fig 5.3B. 
Postprocessing $E_{c c}, E_{c r}$ and $\tau$ were determined before and after adaptation. For comparison with MRT data, strains were determined at the level of the MR slices by interpolation from adjacent nodal points in the FEM mesh. The locations of the MR slices relative to the FEM mesh are shown in Fig 5.3A. Similar to the experimental procedure, strains were computed with respect to begin-ejection and averaged in transmural and circumferential directions.

\subsection{RESULTS}

\subsubsection{MRT experiments}

After triggering on the R-wave, the first tagging and cine frames were captured in the ejection phase. Due to variability in heart rate and tag fading only about $80 \%$ of the cardiac cycle could be imaged. As a result, no strains are determined for the last part of the filling phase and isovolumic contraction phase.

A

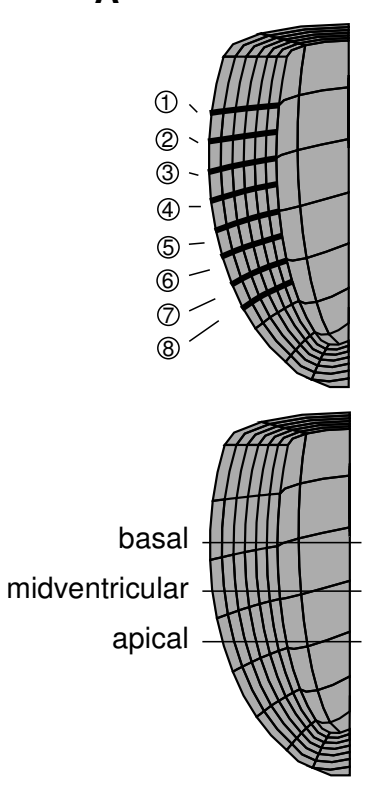

B
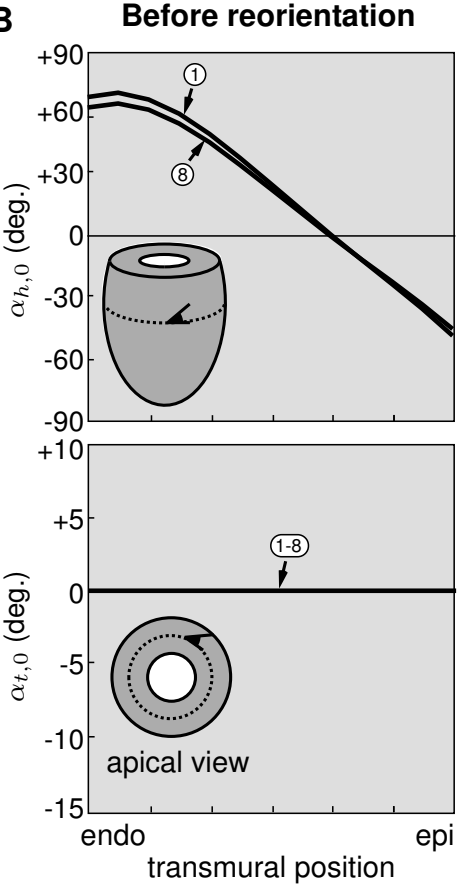

After reorientation
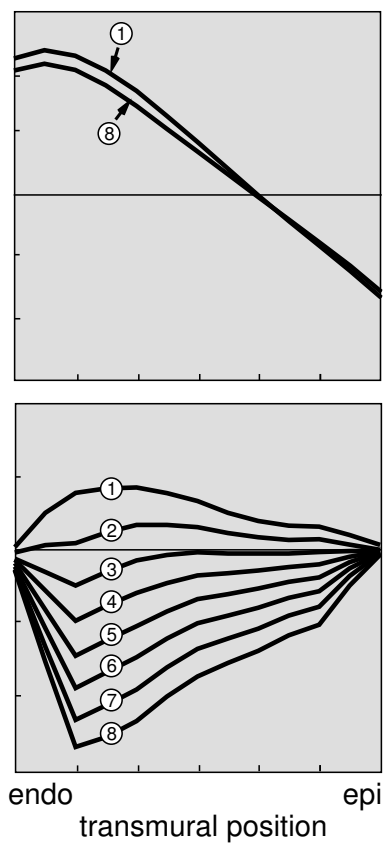

Figure 5.3. A Top: levels for which the transmural variation in helix and transverse angle are visualized. Bottom: positions of the three short-axis slices relative to the finite element mesh. B Top: helix angles $\alpha_{h, 0}$ in the stress-free model geometry. Bottom: transverse angles $\alpha_{t, 0}$. Notice that adaptation mainly affected the transverse angle. 
Fig 5.4 shows that the time course of $E_{c c}, E_{c r}$, and $\tau$ are similar for all three hearts. Typically, $E_{c c}$ decreases during ejection, increases slightly during isovolumic relaxation, and increases further during filling.

In all three slices, $E_{c r}$ decreases equally until one third of the ejection period. Thereafter, $E_{c r}$ continues to decrease near the apex, remains about constant near the equator, and increases near the base. Throughout the isovolumic re-
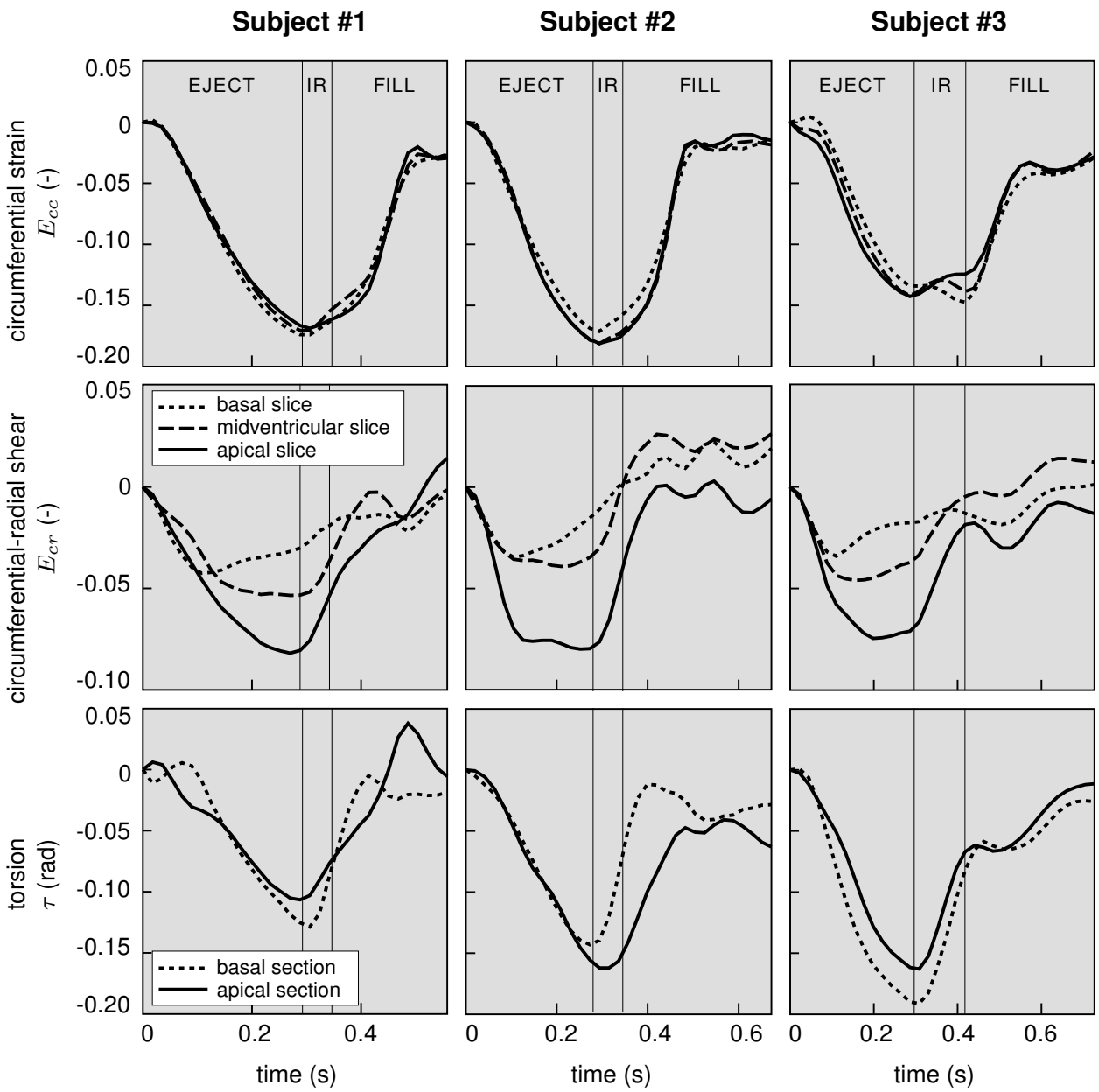

Figure 5.4. Experimentally determined strain for three healthy humans. EJECT=ejection phase; IR=isovolumic relaxation phase; FILL=filling phase. See Fig 5.1A for the position of the slices relative to the ventricular geometry. Torsion is presented for the basal section, between basal and midventricular slice, and the apical section, between midventricular and apical slice. 
laxation phase, $E_{c r}$ increases in all slices, but remains negative. Notice that this negative $E_{c r}$ implies that the subepicardium rotates in clockwise direction with respect to the subendocardium (opposite to deformation shown in Fig 5.2A). The overall amplitude in $E_{c r}$ during the cardiac cycle increases towards the apex, having a value of about 0.08 in the apical slice.

During the ejection phase, $\tau$ decreases reaching a minimum value near the end of ejection. The minimum torsion value differs slightly between the subjects, ranging from about -0.12 in subject one to about -0.17 in subject three. The apexto-base gradient in $\tau$ also differs between subjects. Notice that this negative $\tau$ indicates that during ejection the apex has rotated in counterclockwise direction relative to the base, when viewed from the apex (Fig 5.2B). During isovolumic relaxation, $\tau$ increases by about half the amplitude during ejection. During filling a further, less fast, increase in $\tau$ is observed.

At the last available frame in the filling phase, $E_{c c}, E_{c r}$, and $\tau$ are close to zero. This suggests that during isovolumic contraction changes are limited.

\subsubsection{Mathematical model simulations}

Fig 5.5 shows the time course for $E_{c c}, E_{c r}$, and $\tau$ as simulated with the myofiber orientations before and after adaptation to fiber cross-fiber shear (8).

Before myofiber reorientation Before myofiber reorientation, $E_{c c}$ decreases to about -0.13 during ejection, after which a slight increase is seen during isovolumic relaxation. This increase of $E_{c c}$ is larger towards the apex. $E_{c c}$ increases further during filling to a slightly positive value of about +0.02 . During isovolumic contraction, $E_{c c}$ for all slices returns to zero.

$E_{c r}$ increases to about +0.07 , decreases during the rest of ejection, but remains positive. During isovolumic relaxation and the first part of filling, $E_{c r}$ decreases strongly. Thereafter, $E_{c r}$ continues to decrease slightly. In the isovolumic contraction phase a strong increase in $E_{c r}$ is observed. The overall amplitude of $E_{c r}$ during the cardiac cycle increases towards the apex, having a value of about 0.40 in the apical slice.

During the first third of ejection, $\tau$ increases. This increase is larger in the basal section. Subsequently, a strong decrease is observed to a value of about -0.05 $\mathrm{rad}$ at the end of ejection. During isovolumic relaxation, $\tau$ decreases further; in the basal section $\tau$ decreases more than in the apical section. At begin filling, $\tau$ exhibits a 'hump', yielding a minimum value of about -0.10 rad near the base. Subsequently, $\tau$ increases gradually to about $-0.07 \mathrm{rad}$ at the start of isovolumic relaxation. During isovolumic relaxation $\tau$, increases to zero at begin of ejection of the next cycle. 
After myofiber reorientation After myofiber reorientation, $E_{c c}$ decreases during ejection to about -0.14 . During isovolumic relaxation a small increase is seen that is similar for all slices. During filling $E_{c c}$ becomes positive, followed by a decrease during isovolumic contraction.

During the first third of ejection $E_{c r}$ decreases in the basal slice, while for the midventricular and apical slice an increase is observed. Maximum $E_{c r}$ during ejection is observed at the apex $(+0.06)$. For the remaining part of ejection, $E_{c r}$

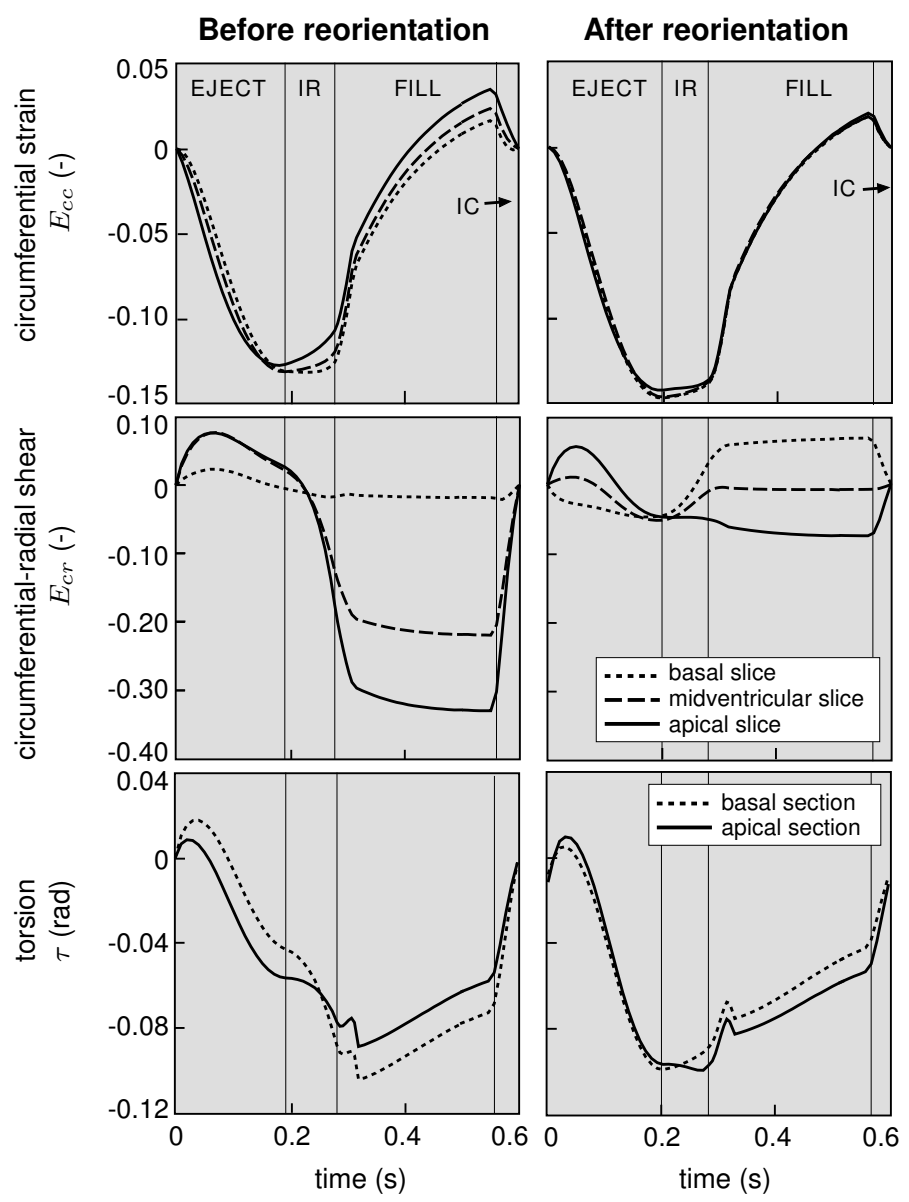

Figure 5.5. Model strains computed with myofiber angles before and after myofiber reorientation. EJECT=ejection phase; IR=isovolumic relaxation phase; FILL=filling phase; $\mathrm{IC}=$ isovolumic contraction phase. See Fig 5.3A for the position of the slices relative to the model geometry. The basal section is between basal and midventricular slice, and the apical section is between midventricular and apical slice. 
decreases in all slices, having a value of about -0.05 at the start of isovolumic relaxation. During isovolumic relaxation $E_{c r}$ increases in the midventricular and basal slice. This increase is more pronounced towards the base. In the apical slice, $E_{c r}$ remains about constant. Consequently, $E_{c r}$ diverges between the slices; at the end of isovolumic relaxation, $E_{c r}$ in the basal, midventricular, and apical slice becomes about $+0.05,0$, and -0.05 , respectively. These differences remain similar during filling. A large change during isovolumic contraction causes $E_{c r}$ in all slices to return to zero at the end of the cardiac cycle.

During the first third of ejection, $\tau$ increases. The apical slice shows a somewhat more pronounced increase. In the remaining part of ejection, $\tau$ decreases to a minimum value of about -0.10 . During isovolumic relaxation apical torsion remains about the same, while basal torsion increases by $0.01 \mathrm{rad}$. During the first part of filling, $\tau$ exhibits a 'hump'. For the remaining part of filling $\tau$ increases gradually to about $-0.05 \mathrm{rad}$. During isovolumic relaxation $\tau$ increases back to zero.

\subsection{DISCUSSION}

In a previous study we estimated myofiber orientations in the cardiac left ventricle with a mathematical model through shear-induced adaption of the myofiber orientations (8). We found that the estimated myofiber orientations were well within the range of experimentally measured ones. In this study, as an additional more critical test for the model, we investigated whether circumferential-radial shear $\left(E_{c r}\right)$ and torsion $(\tau)$, as computed with the adapted myofiber orientation, were realistic as well.

\subsubsection{Comparison between model and experiment}

The MRT data show a consistent time course for circumferential strain $\left(E_{c c}\right)$, $E_{c r}$ and $\tau$ in all three subjects. $E_{c c}$ is kinematically related to cavity volume. Therefore, time course of $E_{c c}$ strongly resembles the time course of LV volume. Sensitivity of $E_{c c}$ on structural properties of the myocardium is relatively low (Fig 5.5). Therefore, it is not surprising that differences in $E_{c c}$ between experimental and simulation data are relatively small.

Kinematic relations between LV volume and $E_{c r}$ and between LV volume and $\tau$ are less strong. Consequently, differences in $E_{c r}$ and $\tau$ between model and experiment are larger than differences in $E_{c c}$. Shear strains $E_{c r}$ and $\tau$ are related to the spatial and temporal pattern variation in tissue anisotropy. Therefore, differences between model and experiment mainly occur during ejection and isovolumic relaxation (Fig 5.4 and Fig 5.5), when tissue anisotropy changes due to myofiber contraction and relaxation. 
In the experiment, $E_{c r}$ becomes negative during ejection with an increase in amplitude towards the apex. During isovolumic relaxation an increase in $E_{c r}$ is observed. At the end of the filling phase, $E_{c r}$ is close to zero, suggesting that during isovolumic contraction changes in $E_{c r}$ are relatively small. Overall amplitude is about 0.08 . In contrast, before myofiber reorientation, the model shows a positive $E_{c r}$ during ejection, followed by a decrease during isovolumic relaxation, yielding a strongly negative $E_{c r}$ at the end of filling. The overall amplitude during ejection is much higher than that in the experiment $(\sim 0.40)$. After myofiber reorientation, the model shows a more realistic overall amplitude of $(\sim 0.10)$. Furthermore, as in the experiment, $E_{c r}$ increases during isovolumic relaxation. However, the apexto-base gradient in $E_{c r}$ is opposite to that in the experiment. Although for the last part of ejection phase $E_{c r}$ is negative, still an unrealistic positive $E_{c r}$ is observed at the begin of ejection.

In the experiment, $\tau$ is minimal at the end of ejection (Bottom row Fig 5.5). After adaptation of the myofiber orientations, a more realistic torsion pattern is obtained, with minimal torsion at the end of ejection, instead of at the start of filling which was the case before adaptation. However, the amplitude is larger in the measurements $(>0.12 \mathrm{rad})$ than in the model $(\sim 0.10 \mathrm{rad})$.

\subsubsection{Myofiber orientation and shear strain}

Our simulations show that through adaptation of the myofiber orientations in response to shear, a more realistic pattern of $E_{c r}$ and $\tau$ can be obtained. To explain these results and the remaining discrepancies between model and experiment, we consider a conceptual model that qualitatively describes the genesis of $E_{c r}$ and $\tau$ (Fig 5.6). LV torsion is determined by the balance of torque generated in the subendo- and subepicardium. Upon shortening of the myofibers in the subendocardial layers, the apex tends to rotate in clockwise direction with respect to the base, when viewing the heart from the apex in apex-to-base direction. Shortening of myofibers in the subepicardial layers tends to rotate the apex in counterclockwise direction. As a consequence a transmural shear load is created. This load results in a circumferential-radial shear deformation. The amplitude and sign of this shear are determined by the shear stiffness of the tissue. In absence of a transmural component in orientation, i.e., with $\alpha_{t}=0$, active shear stiffness is low and mechanical equilibrium is reached with a large positive $E_{c r}$. This explains the overestimation in $E_{c r}$ amplitude as observed in the model before myofiber reorientation. In case of a transmural component in myofiber orientation with a negative $\alpha_{t}$ active force would take part in counterbalancing the shear load. This explains that after myofiber reorientation the amplitude in $E_{c r}$ is significantly reduced. In the experiment, a negative $\tau$ is observed, which implies that the apex rotates counterclockwise with respect to the base. Apparently, the subepicardial layers dominate the subendocardial layers. 


\subsubsection{Study assumptions and limitations}

The remaining discrepancy between shear strains in the mathematical model and those in the experiment may originate from assumptions in the model of cardiac mechanics, in the adaptation model, or in both. From the relation between myofiber orientation and $E_{c r}$ (Fig 5.6), we can identify parameters in the model of cardiac mechanics that limit agreement between model and experiment.

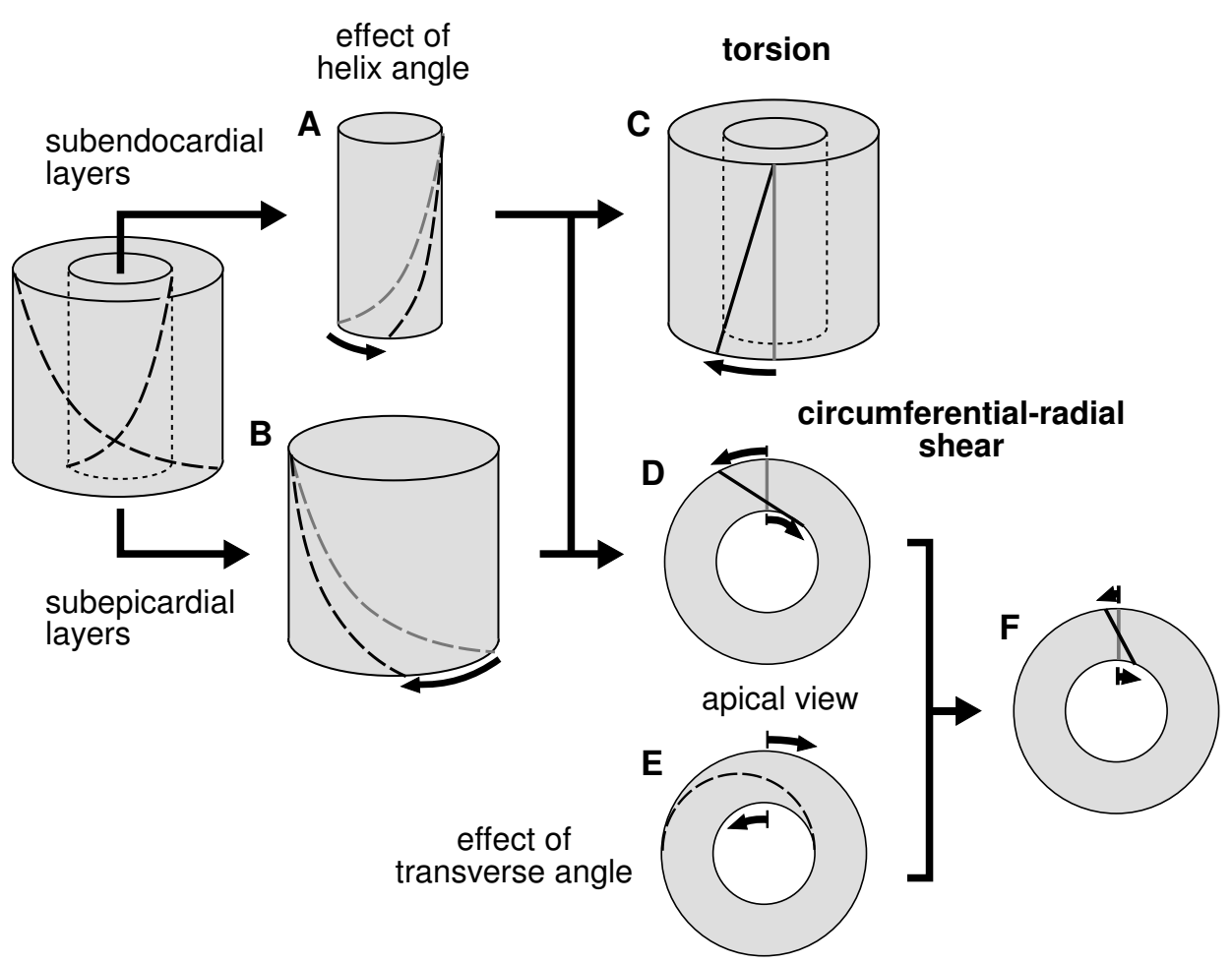

Figure 5.6. Illustration of the influence of myofiber orientations on $\tau$ and $E_{c r}$. While $\tau$ quantifies the axial gradient of average rotation between subsequent slices, $E_{c r}$ quantifies the transmural gradient in rotation within a slice. In absence of a transverse angle, shortening of the myofibers $(--)$ would cause $(\mathbf{A})$ a clockwise apical rotation of the subendocardium, when viewing the apex in apex-to-base direction, and (B) a counterclockwise rotation of the subepicardium. (C) The nett effect of these rotations is apparent through torsion. (D) The resulting transmural difference in rotation creates a transmural shear load yielding a positive $E_{c r}$. (E) In case of a negative transverse angle, active contraction causes a shear load that is opposite to that induced by the transmural difference in rotation. (F) The final shear depends on the balance between the two opposing shear loads. Adapted from Ubbink et al. 2006. 
First, the amplitude of $E_{c r}$ may be reduced by an increase in passive stiffness. The passive stiffness in our model of cardiac mechanics was adopted from Kerckhoffs et al. 2003. Possibly, this stiffness is too low, thereby resulting in the overestimation of $E_{c r}$ amplitude as observed in our model. Second, we assumed passive tissue properties to be transversely isotropic. Biaxial shear experiments suggest passive cardiac tissue to be orthotropic rather than transversely isotropic (5). This orthotropy may potentially affect the transmural coupling, and thus affect the shear strains. However, in a model study, the sensitivity of deformation to the choice of the passive material model was found to be small (16). Third, active force was assumed to be generated in the myofiber direction only. However, biaxial contraction experiments on isolated sheets of myocardium revealed an active force in cross-myofiber direction that was about $40 \%$ of that along myofiber direction (9). This active cross-myofiber force may additionally affect $E_{c r}$ by providing a stronger coupling between the myocardial layers. In the same model as used to assess the sensitivity of wall deformation to orthotropy in passive material stiffness, Usyk et al. 2000 investigated the influence of active cross-fiber stress. They found that active cross-fiber stress significantly affected the shear strains, but agreement with experimental data was not obtained (16). Fourth, we assumed that sarcomere lengths in the externally unloaded LV are homogeneously distributed. In reality, however, the externally unloaded LV exhibits a transmural gradient in sarcomere length with the epicardial sarcomeres being longer than endocardial ones (11). $E_{c r}$ follows from the balance of force between the inner and outer layers of the wall. Consequently, since actively generated myofiber stress is directly related to sarcomere length, a transmural gradient in sarcomere length may alter the shear strains. Kerckhoffs et al. 2003 showed that incorporation of such a transmural gradient in sarcomere length did affect myofiber strains during isovolumic contraction, but changes in myofiber stresses and strains during ejection were limited. Fifth, shear strains may be affected by the transmural differences in mechanical activation of the LV wall. Kerckhoffs et al. 2003 found that the pattern of mechanical activation affects heterogeneity in systolic myofiber strains. Shear strains patterns, however, were not addressed in that study. Finally, absence of a right ventricle and simplifications in the lumped parameter model of the circulation may have affected $E_{c r}$ also.

In the adaptation model, myofiber orientations are determined by the shear strain pattern in the wall. As such, the assumptions described above may indirectly influence the adapted myofiber orientations. It is noted that with more negative transverse angles (=higher amplitude), more realistic (negative) $E_{c r}$ values may be obtained (15). In the same way changes in $E_{c r}$ during isovolumic contraction may be reduced significantly, thus becoming more realistic as well. A future model study is needed to quantitatively assess the sensitivity of shear strains and the resulting myofiber orientations to the material parameters. 
As a final note we consider that in reality, besides myofiber reorientation, other adaptation mechanisms are likely to be active, i.e., not only myofiber orientations, but the above described tissue properties (e.g., passive stiffness, sarcomere lengths) may be the result of adaptation to mechanical load as well. In the future, extension of the adaptation model with additional adaptive mechanisms should be considered.

\subsection{SUMMARY AND CONCLUSION}

In summary, we compared model predicted circumferential-radial shear strain $\left(E_{c r}\right)$ and torsion $(\tau)$ with experimental data as obtained with magnetic resonance tagging (MRT) in three healthy subjects. We found that by adapting myofiber orientation in the model on the basis of fiber cross-fiber shear strain, differences in $E_{c r}$ and $\tau$ between model and experiment could be reduced significantly. However, a complete match could not be obtained on the basis of myofiber reorientation alone.

\section{REFERENCES}

1. Axel L, Dougherty $L$ (1989) MR imaging of motion with spatial modulation of magnetization. Am J Physiol Heart Circ Physiol 171:841-845.

2. Bovendeerd PH, Huyghe JM, Arts T, van Campen DH, Reneman RS (1994) Influence of endocardial-epicardial crossover of muscle fibers on left ventricular wall mechanics. J Biomech 27:941-951.

3. Bovendeerd PH, Rijcken J, van Campen DH, Schoofs AJG, Nicolay K, Arts T (1998) Optimization of left ventricular muscle fiber orientation. In: Pedersen P, Bendsøe MP (Eds) Proc IUTAM Symposium on Synthesis on Bio Solid Mechanics, Copenhagen, Denmark. pp. 285-295 (ISBN13: 978-0-792-35615-8).

4. Delhaas T, Kroon W, Decaluwe W, Rubbens M, Bovendeerd P, Arts T (2008) Structure and torsion of the normal and the situs inversus totalis cardiac left ventricle. I. Experimental data in humans. Am J Physiol Heart Circ Physiol 295:H197-H201.

5. Dokos S, Smaill BH, Young AA, LeGrice IJ (2002) Shear properties of passive ventricular myocardium. Am J Physiol Heart Circ Physiol 283:H2650-H2659.

6. Geerts L, Bovendeerd P, Nicolay K, Arts T (2002) Characterization of the normal cardiac myofiber field in goat measured with MR-diffusion tensor imaging. Am J Physiol Heart Circ Physiol 283:H139-H145.

7. Kerckhoffs RC, Bovendeerd PH, Kotte JC, Prinzen FW, Smits K, Arts T (2003) Homogeneity of cardiac contraction despite physiological asynchrony of depolarization: a model study. Ann Biomed Eng 31:536-547.

8. Kroon W, Delhaas T, Bovendeerd P, Arts T (2008) Computational analysis of the myocardial structure: Adaptation of cardiac myofiber orientations through deformation. Med Imag Anal doi:10.1016/j.media.2008.06.015.

9. Lin DH, Yin FC (1998) A mulitaxial constitutive law for mamalian left ventricular myocardium in steady-state barium contracture or tetanus. J Biomech Eng 120:504-517. 
10. MacKenna D, Summerour SR, Villarreal FJ (2000) Role of mechanical factors in regulating fibroblast function and extracellular matrix synthesis. Cardiovasc Res 46:257-263.

11. Rodriguez EK, Omens JH, Waldman LW, McCulloch AD (1993) Effect of residual stress on transmural sarcomere length distributions in rat left ventricle. Am J Physiol Heart Circ Physiol 264:H1048-H1056.

12. Sadoshima J, Izumo S (1997) The cellular and molecular response of cardiac myocytes to mechanical stress. Ann Rev Physiol 59:551-571.

13. Simpson DG, Majeski M, Borg TK, Terracio L (1999) Regulation of cardiac myocyte protein turnover and myofibrillar structure in vitro by specific directions of stretch. Circ Res 85 :e59e69.

14. Spinale FG (2007) Myocardial matrix remodeling and the matrix metalloproteinases: influence on cardiac form and function. Physiol Rev 87:1285-1342.

15. Ubbink SW, Bovendeerd PH, Delhaas T, Arts T, van de Vosse FN (2006) Towards model based analysis of cardiac MR tagging data: relation between left ventricular shear strain and myofiber orientation. Med Imag Anal 10:632-641.

16. Usyk TP, Mazhari R, McCulloch AD (2000) Effect of laminar orthotropic myofiber architecture on regional stress and strain in the canine left ventricle. J Elast 61:143-164. 



\section{Computational modeling of volumetric soft tissue growth Application to the cardiac left ventricle}

Wilco Kroon ${ }^{2}$, Tammo Delhaas ${ }^{1,2}{\text {, Theo } \text { Arts }^{3} \text { and Peter Bovendeerd }}^{4}$

Department of ${ }^{1}$ Pediatrics, ${ }^{2}$ Physiology and ${ }^{3}$ Biophysics, Cardiovascular Research Institute Maastricht, University Hospital Maastricht, The Netherlands; and ${ }^{4}$ Department of Biomedical Technology, Eindhoven University of Technology, Eindhoven, The Netherlands.

Biomechan Model Mechanobiol. Doi:10.1007/s10237-008-0136-z, 2008 


\begin{abstract}
To investigate stimulus-response relations in growth and remodeling (G\&R) of cardiac tissue, this study aims to develop a method to simulate 3D inhomogeneous volumetric growth. Growth is regarded as a deformation that is decomposed into a plastic component which describes unconstrained growth and an elastic component to satisfy continuity of the tissue after growth. In current growth models, a single reference configuration is used that remains fixed throughout the entire growth process. However, considering continuous turnover to occur together with growth, such a fixed reference is unlikely to exist in reality. Therefore, we investigated the effect of tissue turnover on growth by incrementally updating the reference configuration. With both a fixed reference and an updated reference, strain-induced cardiac growth in magnitude of $30 \%$ could be simulated. With an updated reference, the amplitude of the stimulus for growth decreased over time. However, with a fixed reference this amplitude increased. We conclude that, when modeling volumetric growth, the choice of the reference configuration is of great importance for the computed growth.
\end{abstract}

\title{
6.1 INTRODUCTION
}

Over the years several finite element (FE) models of cardiac mechanics have been developed to study pump function in relation to the three-dimensional (3D) geometrical, passive, active, and anisotropic properties of the myocardium $(3,4$, $7,13,16,24,27)$. Tissue properties, however, are not constant over time as the tissue exhibits growth and remodeling (G\&R) in response to (changes in) mechanical loading $(5,6,8-10,15,21,22)$. The clinically most evident examples are left ventricular (LV) hypo- or hypertrophy in response to hemodynamic underor overloading of the LV, respectively. Furthermore, local changes in mechanical loading, as induced by asynchronous contraction, have been shown to result in asymmetric wall thickening (28). The details on the type of stimulus and the local response, however, still remain unclear $(11,17)$. Extension of models of cardiac mechanics with features of G\&R may provide additional insight in adaptation by allowing quantitative testing of hypotheses regarding the stimulus-response relation. Subsequently, thus extended models may be used to estimate the long-term outcome of clinical interventions that cause chronic changes in load, such as cardiac re-synchronization therapy.

Recently, 3D FE models have been developed that enable computation of volumetric growth in patient specific geometries $(1,14,18,20)$. In these models, volumetric growth is regarded as a deformation that potentially changes the externally unloaded shape, volume, and state of stress of the tissue $(19,23,25)$. Internal stresses are constitutively related to the growth deformation via an ini- 
tial stress-free configuration of the tissue that remains fixed throughout the entire growth process. As such, growth remains dependent on the initial tissue configuration.

In an alternative approach, the tissue has been considered as a mixture of constituents, each of which exhibits continuous turnover (12). An implication of this turnover is that a certain initial configuration becomes irrelevant once all initial tissue constituents have been renewed. From this argument, constitutive laws used in growth models to relate the internal stresses to the growth deformation should not be related to a fixed reference configuration, but rather to a configuration that evolves together with growth.

The aim of the present study is two-fold. The first aim is to include volumetric growth in an existing 3D model of cardiac mechanics. The second aim is to investigate the effect of using a fixed or an evolving stress-free reference on progression of volumetric growth. Both approaches are implemented in a 3D FE framework.

\subsection{METHOD}

\subsubsection{Growth during a single time increment}

Assuming density of soft tissue to be constant, growth is modeled by a change of volume. As shown in Fig 6.1, a growth increment from time $t=t_{0}$ to time $t=t_{0}+\Delta t$ is simulated by deforming the stress-free tissue configuration $\mathcal{B}_{0}$ to the externally unloaded, not necessarily internally stress-free, grown configuration $\mathcal{B}_{g}$ in three steps.

Step 1: Computation of the growth stimulus In the first step, the externally loaded tissue configuration $\mathcal{B}_{l}$ is determined. Deformation is quantified by the load deformation gradient tensor $\boldsymbol{F}_{l}$, which is related to tissue displacements $\vec{u}_{l}$ through:

$$
\boldsymbol{F}_{l}=\left(\vec{\nabla}_{0} \vec{u}_{l}\right)^{T}+\boldsymbol{I}
$$

with $\vec{\nabla}_{0}$ the spatial gradient operator with respect to the stress-free configuration $\mathcal{B}_{0}$ and $\boldsymbol{I}$ the identity tensor.

Under external loading conditions, Cauchy stress $\sigma$ in the tissue is constitutively related to deformation $\boldsymbol{F}_{l}$ by a strain energy density function $W_{l}$ :

$$
\boldsymbol{\sigma}\left(\boldsymbol{F}_{l}\right)=\frac{1}{\operatorname{det}\left(\boldsymbol{F}_{l}\right)} \boldsymbol{F}_{l} \cdot \frac{\partial W\left(\boldsymbol{E}_{l}\right)}{\partial \boldsymbol{E}_{l}} \boldsymbol{F}_{l}^{T}
$$

where $\boldsymbol{E}_{l}=\frac{1}{2}\left(\boldsymbol{F}_{l}^{T} \cdot \boldsymbol{F}_{l}-\boldsymbol{I}\right)$ is the Green-Lagrangian strain due to external loading. Displacements are computed from balance of linear momentum, which under 


\section{Chapter 6}

the assumption of negligible body and inertial forces reads:

$$
\vec{\nabla}_{l} \cdot \boldsymbol{\sigma}\left(\boldsymbol{F}_{l}\right)=\overrightarrow{0} \text { on } \mathcal{B}_{l}
$$

where $\vec{\nabla}_{l}$ denotes the spatial gradient operator with respect to $\mathcal{B}_{l}$. The problem definition is completed with natural and essential boundary conditions along deformed surface parts $\partial B_{l, n}$ and $\partial B_{l, e}$, respectively:

$$
\begin{aligned}
\boldsymbol{\sigma}\left(\boldsymbol{F}_{l}\right) \cdot \vec{n} & =\vec{t}_{p} \text { on } \partial \mathcal{B}_{l, n} \\
\vec{u}_{l} & =\vec{u}_{p} \text { on } \partial \mathcal{B}_{l, e}
\end{aligned}
$$

with $\vec{t}_{p}$ a prescribed traction, $\vec{u}_{p}$ a prescribed displacement, and $\vec{n}$ the unit outer normal of the deformed surface.

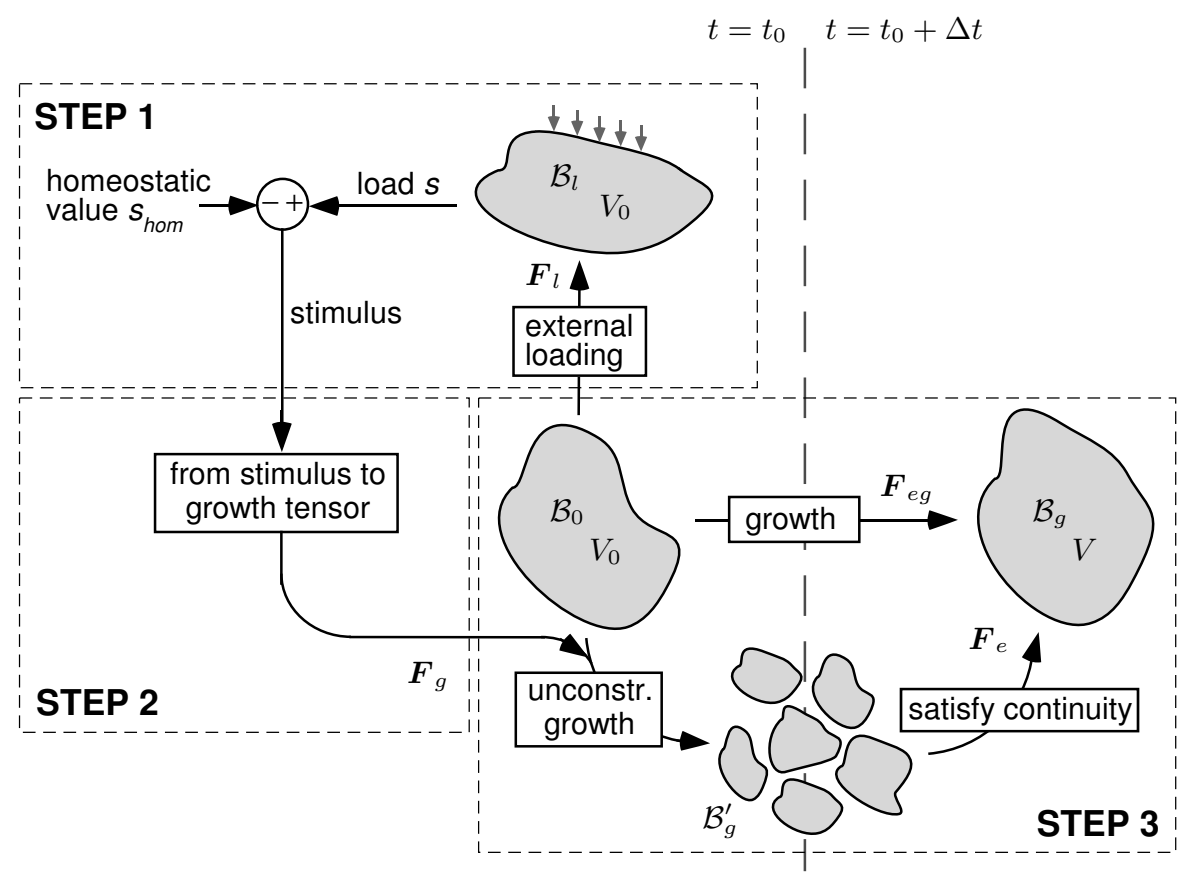

Figure 6.1. Schematic description of a growth increment. Simulation of a growth increment from time $t=t_{0}$ to time $t=t_{0}+\Delta t$ involves three steps. In step 1 , the externally unloaded configuration $\mathcal{B}_{0}$ with volume $V_{0}$ is externally loaded, resulting in configuration $\mathcal{B}_{l}$. In this configuration a load measure $s$ is extracted and the growth stimulus is determined from deviation of $s$ from a homeostatic value $s_{\text {hom }}$. In step 2, the stimulus is translated into a growth tensor $\boldsymbol{F}_{g}$ that describes the local unconstrained growth of the unloaded tissue to the intermediate configuration $\mathcal{B}_{g}^{\prime}$. To satisfy continuity of the grown tissue, an additional deformation $\boldsymbol{F}_{e}$ is required, that generates internal stresses. In step 3, $\boldsymbol{F}_{g}$ is used to compute the combined growth deformation $\boldsymbol{F}_{e g}=\boldsymbol{F}_{e} \cdot \boldsymbol{F}_{g}$ from equilibrium of linear momentum, yielding grown configuration $\mathcal{B}_{g}$ with volume $V$. 
Next, a scalar load measure $s$ is extracted from $\mathcal{B}_{l}$. Similar to Taber 1998, we assume growth to be stimulated if the load $s$ differs from its homeostatic (i.e., target) value $s_{\text {hom }}$, i.e.:

$$
\text { stimulus }=\text { function }\left(s-s_{\text {hom }}\right)
$$

Step 2: Translation of the stimulus to a growth tensor In a second step, the stimulus is translated into an incremental growth deformation tensor $\boldsymbol{F}_{g}$ that describes unconstrained tissue growth. Local volume changes during growth are mathematically specified by the determinant of $\boldsymbol{F}_{g}$ :

$$
\operatorname{det}\left(\boldsymbol{F}_{g}\right)=\frac{V}{V_{0}}
$$

with $V_{0}$ and $V$ the local tissue volumes before and after the growth increment. The isochoric part $\tilde{\boldsymbol{F}}_{g}=\operatorname{det}\left(\boldsymbol{F}_{g}\right)^{-\frac{1}{3}} \boldsymbol{F}_{g}$ specifies the preferred direction in which the new tissue is deposited. In this study, without loss of generality of the kinematic description, new tissue constituents are assumed to be deposited in all directions equally (isotropic growth) and $\boldsymbol{F}_{g}$ takes the form:

$$
\boldsymbol{F}_{g}=\left(\frac{V}{V_{0}}\right)^{\frac{1}{3}} \boldsymbol{I}
$$

The idea that a supra-homeostatic load will lead to a net increase of volume, whereas a sub-homeostatic load will lead to a decrease of volume, is phenomenologically described by:

$$
\dot{V}=\beta V\left(s-s_{\text {hom }}\right)
$$

with $\beta$ a rate constant. Linearization with respect to volume $V_{0}$ at the start of the growth increment yields:

$$
\frac{V}{V_{0}}=\beta\left(s-s_{\text {hom }} \Delta t\right)+1
$$

Consequently, for the incremental growth deformation tensor it holds:

$$
\boldsymbol{F}_{g}=\left[\beta\left(s-s_{h o m} \Delta t\right)+1\right]^{\frac{1}{3}} \boldsymbol{I}
$$

Step 3: Computation of growth displacements In a third step, the growth tensor $\boldsymbol{F}_{g}$ is translated into growth displacements $\vec{u}_{e g}$. Deformation according to $\boldsymbol{F}_{g}$ leads to the fictitious intermediate configuration $\mathcal{B}_{g}^{\prime}$ in which local tissue parts have expanded (figure 6.1). Generally, an additional deformation is required to ensure that locally grown tissue parts form an intact tissue configuration $\mathcal{B}_{g}$. This deformation is quantified by $\boldsymbol{F}_{e}$. Consequently, a tensor $\boldsymbol{F}_{e g}$ is defined that transforms the unloaded configuration $\mathcal{B}_{0}$ into the grown configuration $\mathcal{B}_{g}$ :

$$
\boldsymbol{F}_{e g}=\boldsymbol{F}_{e} \cdot \boldsymbol{F}_{g}
$$


Change in tissue volume is related to $\boldsymbol{F}_{g}$ only, i.e., $\boldsymbol{F}_{e}$ is isochoric. Growth displacements are related to $\boldsymbol{F}_{e g}$ according to:

$$
\boldsymbol{F}_{e g}=\left(\vec{\nabla}_{0} \vec{u}_{e g}\right)^{T}+\boldsymbol{I}
$$

Generally, part $\boldsymbol{F}_{g}$ is assumed to be plastic and stresses are only generated by the elastic part $\boldsymbol{F}_{e}(1,14,18-20,23,25)$. In our model, the growth-induced Cauchy stress $\boldsymbol{\sigma}_{g}\left(\boldsymbol{F}_{e}\right)$ is related to a strain energy density function $W_{g}$ through:

$$
\boldsymbol{\sigma}_{g}\left(\boldsymbol{F}_{e}\right)=\frac{1}{\operatorname{det}\left(\boldsymbol{F}_{e}\right)} \boldsymbol{F}_{e} \cdot \frac{\partial W_{g}\left(\boldsymbol{E}_{e}\right)}{\partial \boldsymbol{E}_{e}} \cdot \boldsymbol{F}_{e}^{T}
$$

with $\boldsymbol{E}_{e}=\frac{1}{2}\left(\boldsymbol{F}_{e}^{T} \cdot \boldsymbol{F}_{e}-\boldsymbol{I}\right)$. Growth displacements are computed from balance of linear momentum, which is now given by:

$$
\vec{\nabla}_{g} \cdot \boldsymbol{\sigma}_{g}\left(\boldsymbol{F}_{e}\right)=\overrightarrow{0} \text { on } \mathcal{B}_{g}
$$

where $\vec{\nabla}_{g}$ denotes the spatial gradient operator with respect to the intact grown configuration $\mathcal{B}_{g}$. Growth is assumed to alter the externally unloaded (tractionfree) tissue configuration. To model this situation, boundary conditions are specified according to:

$$
\begin{aligned}
\boldsymbol{\sigma}_{g}\left(\boldsymbol{F}_{e}\right) \cdot \vec{n} & =\overrightarrow{0} \quad \text { on } \partial \mathcal{B}_{g, n} \\
\vec{u} & =\vec{u}_{p} \text { on } \partial \mathcal{B}_{g, e}
\end{aligned}
$$

\subsubsection{Cumulative growth}

Generally, after one growth increment, the initial unloaded configuration $\mathcal{B}_{0}$ is deformed by $\boldsymbol{F}_{e g, 1}$ to form the new externally unloaded tissue configuration $\mathcal{B}_{g, 1}$ (Fig 6.2), where the subscript is added to indicate the increment number. Next, a new growth increment is started by loading configuration $\mathcal{B}_{g, 1}$, resulting in externally loaded configuration $\mathcal{B}_{l, 1}$ where a stimulus is computed and translated into a new growth deformation $\boldsymbol{F}_{g, 2}$. Subsequently, $\boldsymbol{F}_{g, 2}$ is used to determine the new configuration $\mathcal{B}_{g, 2}$, which is related to $\mathcal{B}_{g, 1}$ through $\boldsymbol{F}_{\text {eg,2 }}$. As can be derived from Fig 6.2, after $n$ growth increments $\mathcal{B}_{0}$ is cumulatively deformed into $\mathcal{B}_{g, n}$ according to $\boldsymbol{F}_{e g}^{(n)}$ which is given by:

$$
\begin{aligned}
\boldsymbol{F}_{e g}^{(n)} & =\boldsymbol{F}_{e g, n} \cdot \boldsymbol{F}_{e g, n-1} \cdots \boldsymbol{F}_{e g, 1} \\
& =\prod_{i=1}^{n} \boldsymbol{F}_{e g, i}
\end{aligned}
$$

To asses the effect of using a fixed or an updated stress-free configuration, we distinguish between two approaches to compute the cumulative growth tensor (Fig 6.2A,B). 
Fixed reference configuration Fig 6.2A schematically shows the commonly used approach in which the stress-free reference configuration $\mathcal{B}_{0}$ remains fixed throughout the growth simulation $(1,14,18,20)$. In this approach, internal stresses after increment $n$ are related to $\boldsymbol{F}_{e, n}$ as given by:

$$
\boldsymbol{F}_{e, n}=\boldsymbol{F}_{e g}^{(n)} \cdot\left(\boldsymbol{F}_{g}^{(n)}\right)^{-1}
$$

with $\boldsymbol{F}_{g}^{(n)}$ the cumulative unconstrained tissue growth, that is given by:

$$
\boldsymbol{F}_{g}^{(n)}=\prod_{i=1}^{n} \boldsymbol{F}_{g, i}
$$

In this approach, the cumulative grown deformation $\boldsymbol{F}_{e g}^{(n)}$ is directly determined from the balance of linear momentum in Eq 6.15 by using $\boldsymbol{F}_{g}^{(n)}$ as input with stresses related to $\boldsymbol{F}_{e, n}$. Thus, internal stresses in the grown configuration remain dependent on the initial configuration $\mathcal{B}_{0}$ through $\boldsymbol{F}_{e g}^{(n)}$.

Updated reference configuration In this case, growth-induced internal stresses are assumed to relax due to tissue turnover. To describe this behavior the stressfree reference configuration is updated after each growth increment using the

\section{fixed reference}
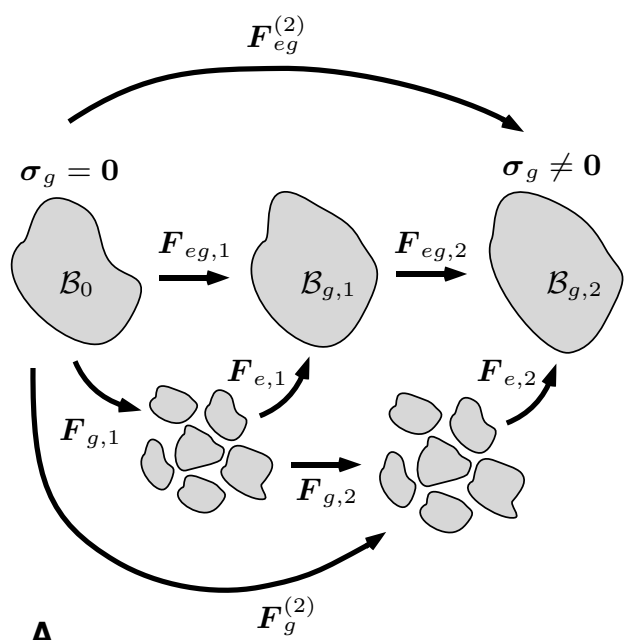

updated reference
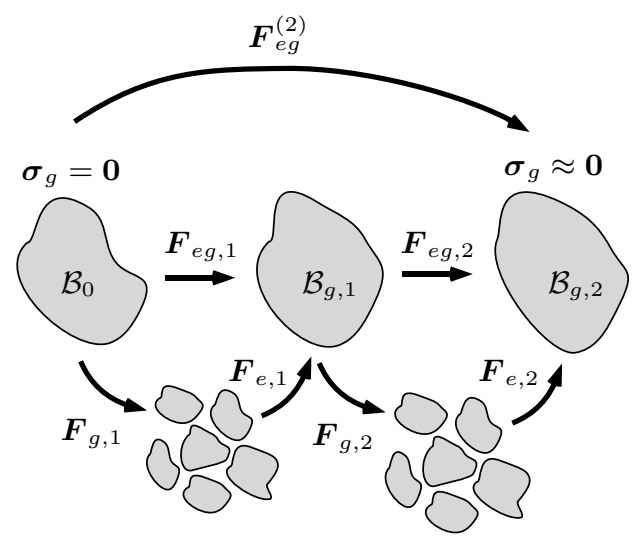

B

Figure 6.2. Configurations and deformations involved during successive load-induced growth increments. A The stress-free reference tissue configuration $\mathcal{B}_{0}$ remains fixed and internal stresses accumulate. B The stress-free reference configuration $\mathcal{B}_{0}$ is updated after each growth increment. Consequently, stresses will be present in the grown tissue, but they will not accumulate. 
computed incremental tissue displacements (Fig 6.2B). Consequently, internal stress in the grown configuration is only dependent on the last grown configuration $\mathcal{B}_{g, n-1}$ through $\boldsymbol{F}_{e g, n}$. Now, for the elastic deformation $\boldsymbol{F}_{e, n}$ it holds:

$$
\boldsymbol{F}_{e, n}=\boldsymbol{F}_{e g, n} \cdot \boldsymbol{F}_{g, n}^{-1}
$$

In this approach, the incremental growth deformation $\boldsymbol{F}_{e g, n}$ is computed from the balance of linear momentum using the incremental unconstrained growth tensor $\boldsymbol{F}_{g, n}$ as input. The cumulative deformations $\boldsymbol{F}_{e g}^{(n)}$ and $\boldsymbol{F}_{g}^{(n)}$ are then determined by the incremental deformations according to Eq 6.16 and Eq 6.17, respectively.

\subsubsection{Application to the cardiac left ventricle}

Geometry We illustrate both approaches by simulating growth in the cardiac left ventricle (LV). The unloaded LV wall is represented by a truncated ellipsoid with a cavity and wall volume of $44 \mathrm{ml}$ and $120 \mathrm{ml}$, respectively (Fig 6.3A). Both are representative volumes for the dog LV (3).

Strain energy density function Under loading conditions, the passive cardiac tissue is strongly non-linear and anisotropic due to the fibrous structure. To model this behavior, we use the strain energy density function $W_{l}$ as proposed by Kerckhoffs et al. 2003:

$$
W_{l}=W_{m}+W_{f}+W_{v}
$$

with

$$
\begin{aligned}
W_{m} & =a_{0}\left[e^{a_{1} \operatorname{tr}\left(\boldsymbol{E}_{l}^{T} \cdot \boldsymbol{E}_{l}\right)}-1\right] \\
W_{f} & =a_{2}\left[e^{a_{3} E_{f f}^{2}}-1\right] \\
W_{v} & =a_{4}\left[\operatorname{det}\left(\boldsymbol{F}_{l}\right)^{2}-1\right]^{2}
\end{aligned}
$$

where objectivity of stress is ensured by choosing $W_{l}$ as a function of $\boldsymbol{E}_{l}$. Part $W_{m}$ describes the isotropic response due to extracellular matrix deformation, while $W_{f}$ describes additional stiffness in myofiber direction. The Green-Lagrangian myofiber strain $E_{f f}$ is specified by $E_{f f}=\vec{e}_{f, 0} \cdot \boldsymbol{E}_{l} \cdot \vec{e}_{f, 0}$. Near-incompressible behavior is enforced through $W_{v}$. Material parameter values $a_{0}=0.5 \mathrm{kPa}, a_{1}=3.0$ $\mathrm{kPa}, a_{2}=0.01 \mathrm{kPa}$ and $a_{3}=60.0 \mathrm{kPa}$ were obtained from Kerckhoffs et al. 2003. The bulk modulus $a_{4}$ was set to $5.0 \mathrm{kPa}$.

Myofiber orientation Myofiber orientation $\vec{e}_{f, 0}$ in the externally unloaded LV is quantified by helix angle $\alpha_{h, 0}$ with respect to a prolate ellipsoidal coordinate 
system:

$$
\vec{e}_{f, 0}=\cos \left(\alpha_{h, 0}\right) \vec{e}_{c, 0}+\sin \left(\alpha_{h, 0}\right) \vec{e}_{l, 0}
$$

where $\vec{e}_{c, 0}$ and $\vec{e}_{l, 0}$ denote the circumferential and the longitudinal direction in the unloaded geometry, respectively (Fig 6.3A). Initially, the myofiber angle $\alpha_{h, 0}$ varies linearly in transmural direction from $+60^{\circ}$ at the endocardium to $-60^{\circ}$ at the epicardium. During growth, new tissue is assumed to locally have the same myofiber angle as that in the existing tissue. This implies that transmural heterogeneity in tissue growth affects the transmural course of the myofiber angle.

Stimulus and growth Growth is assumed to be triggered by a deviation of enddiastolic myofiber strain from a homeostatic value $s_{\text {hom }}=0.13$. This homeostatic value roughly resembles myofiber strain during the filling phase in the healthy LV. End-diastolic myofiber strain $s$ is given by:

$$
s=\sqrt{2 E_{f f}+1}-1
$$

The end-diastolic situation was simulated by imposing a surface traction $\vec{t}_{p}=$ $-p \vec{n}$, with $\vec{n}$ the unit outer normal of the deformed surface and $p$ the cavity pressure of $1 \mathrm{kPa}$.

During growth, the product $\beta \Delta t$ in Eq 6.10 was set to 1. The strain energy density function was taken the same as during external loading, i.e., $W_{g}=W_{l}$.
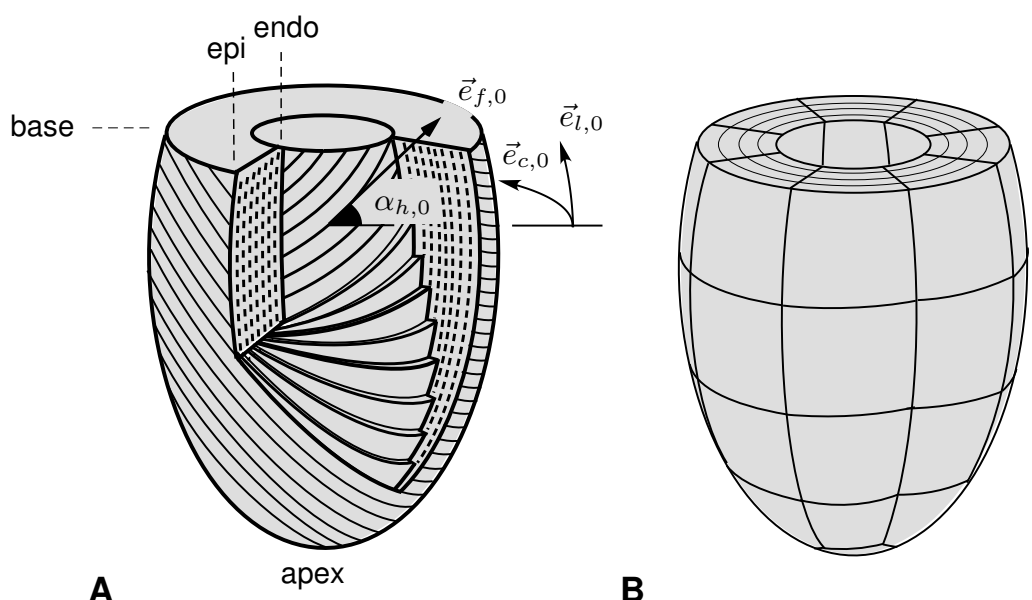

B

Figure 6.3. Computational geometry of the left ventricle (LV). A The externally unloaded LV geometry is represented by a truncated ellipsoid. In this geometry, myofiber directions $\vec{e}_{f, 0}$ are quantified by angles $\alpha_{h, 0}$ with respect to circumferential direction $\vec{e}_{c, 0}$. endo=endocardium, epi=epicardium. B Finite element mesh containing triquadratic hexahedral elements consisting of 27 nodal points with 3 displacement degrees of freedom each. 
Simulations and analysis Using both a fixed and an updated reference configuration to simulate growth, we assessed volume changes, changes in end-diastolic strains, and changes in myofiber angles. The number of growth increments was chosen such that for both simulations, volume changed by about $30 \%$. Change in unloaded tissue volume was computed according to:

$$
\left(\operatorname{det}\left(\boldsymbol{F}_{e g}^{(n)}\right)-1\right) \times 100 \%
$$

Furthermore, to asses directionality of growth, we divided change in tissue volume into change of myofiber length as computed according to:

$$
\left(\lambda_{e g, f}-1\right) \times 100 \%
$$

and change of area perpendicular to the myofiber axis (cross-myofiber area) as computed from:

$$
\left(\frac{\operatorname{det}\left(\boldsymbol{F}_{e g}^{(n)}\right)}{\lambda_{e g, f}}-1\right) \times 100 \%
$$

where myofiber growth stretch ratio $\lambda_{e g, f}$ is given by:

$$
\lambda_{e g, f}=\sqrt{\vec{e}_{f, 0} \cdot C_{e g}^{(n)} \cdot \vec{e}_{f, 0}}
$$

with $\boldsymbol{C}_{e g}^{(n)}=\boldsymbol{F}_{e g}^{(n)^{T}} \cdot \boldsymbol{F}_{e g}^{(n)}$ the right Cauchy growth strain.

\subsubsection{Numerical treatment}

At each time increment, tissue displacements due to external loading or growth are determined by solving the balance of linear momentum in Eq 6.3 and Eq 6.14 with a Galerkin-type of finite element method (2), as implemented in the commercial package SEPRAN (SEPRA, Leidschendam, The Netherlands). Prescribed displacements $\vec{u}_{p}$ are identical for the loading and growth step; basal movement is restricted in apex-to-base direction, while rotation of the endocardium with respect to the epicardium is allowed. Switching from computation of displacements during loading $\left(\vec{u}_{l}\right)$ to computation of displacements during growth $\left(\vec{u}_{e g}\right)$ involves a change in traction $\vec{t}_{p}$ by switching between cavity pressure $p$ from $1 \mathrm{kPa}$ to 0 $\mathrm{kPa}$, respectively.

A modified Newton-Raphson method was employed to linearize balance of linear momentum with respect to the stress-free reference configuration (total Lagrangian formulation). Spatial discretization occurred with triquadratic hexahedral elements, while spatial integration was conducted with a Newton-Cotes scheme.

The mesh of the initial unloaded stress-free reference configuration is visualized in figure Fig 6.3B. For the fixed reference approach, the finite element mesh remains the same for each growth increment. For the updated reference approach, 
the mesh is updated after a growth increment by displacing the nodal points by the incremental growth displacements $\vec{u}_{e g}$, while neglecting the internal stresses. This results in a new stress-free mesh.

\subsection{RESULTS}

Fig 6.4A shows an inhomogeneous distribution of end-diastolic myofiber strain after the first loading step, as visualized in the initial unloaded geometry. Enddiastolic myofiber strains are higher at the subendocardium than at the subepicardium.

Fig 6.4B and Fig 6.4C show the total amount of volume change, together with the change in geometry as obtained with a fixed reference and an updated reference, respectively. With both cases significant changes in volume could be obtained with a magnitude of $30 \%$. However, to achieve this change with an updated reference approach about twice as much increments were needed.

As growth progressed and the reference configurations in both approaches started to diverge, end-diastolic strain became different also. Fig 6.5 provides a

before growth

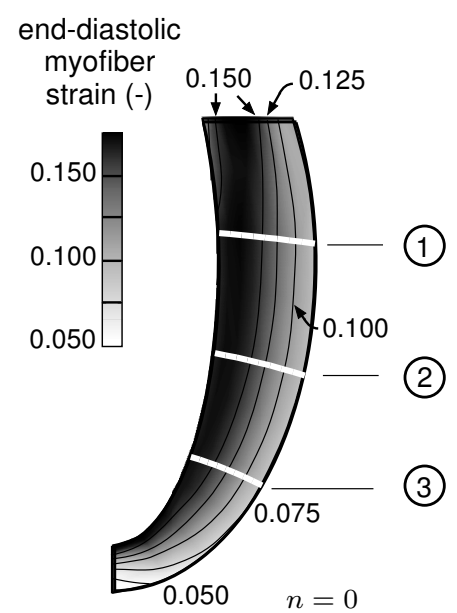

A after growth, fixed reference

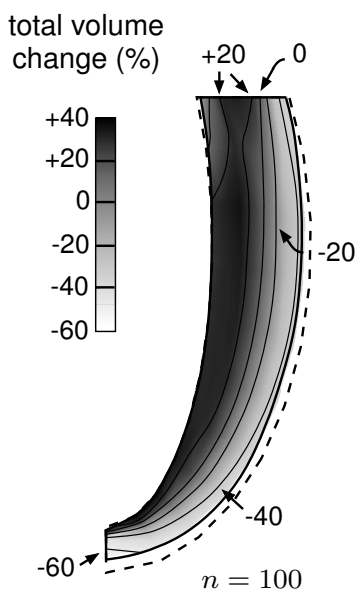

B
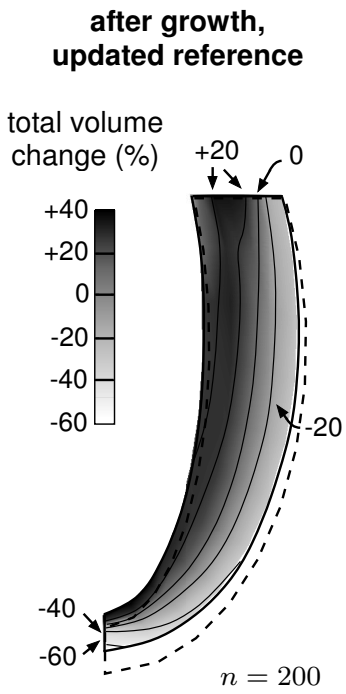

C

Figure 6.4. Load-induced growth in the cardiac LV: global volume and shape changes. A Initial myofiber strain at end of diastole (cavity pressure $p=1 \mathrm{kPa}$ ). B-C Cumulative volume changes in the grown geometry. The dashed contour indicates the initial geometry. The number of increments $n=t /(\beta \Delta t)$. Simulation end-times were selected upon volumetric growth being in magnitude of $30 \%$. Since the evolution of the stimulus differed between the two simulations, both have different end-times. 


\section{Chapter 6}

more detailed view of the transmural distributions of tissue growth, end-diastolic strain, and helix angles before and after growth at three different longitudinal levels. Fig $6.5 \mathrm{~A}$ shows that the pattern of local volume change is similar for the two approaches. When local volume change is divided into change in cross-myofiber

....... before growth

-..- after growth, fixed reference

_ after growth, updated reference

volume change (\%)

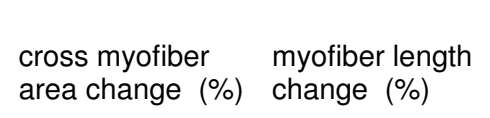

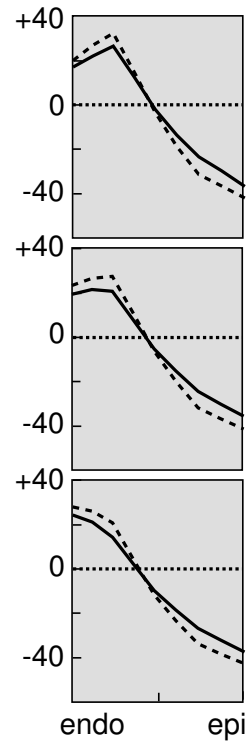

A

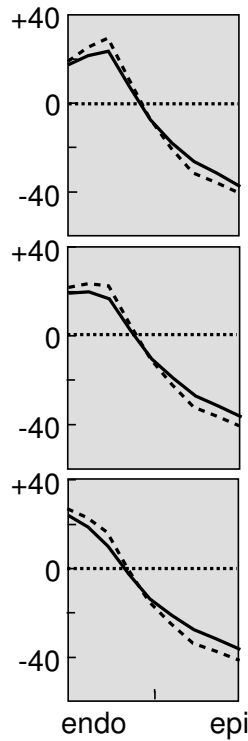

B

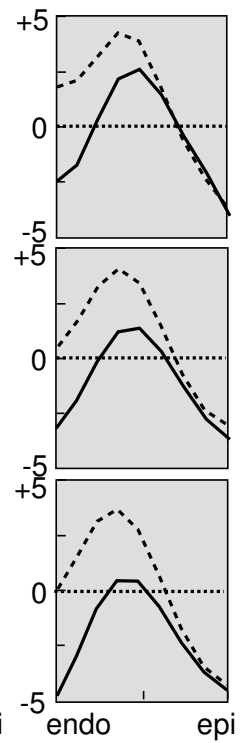

C homeostatic level

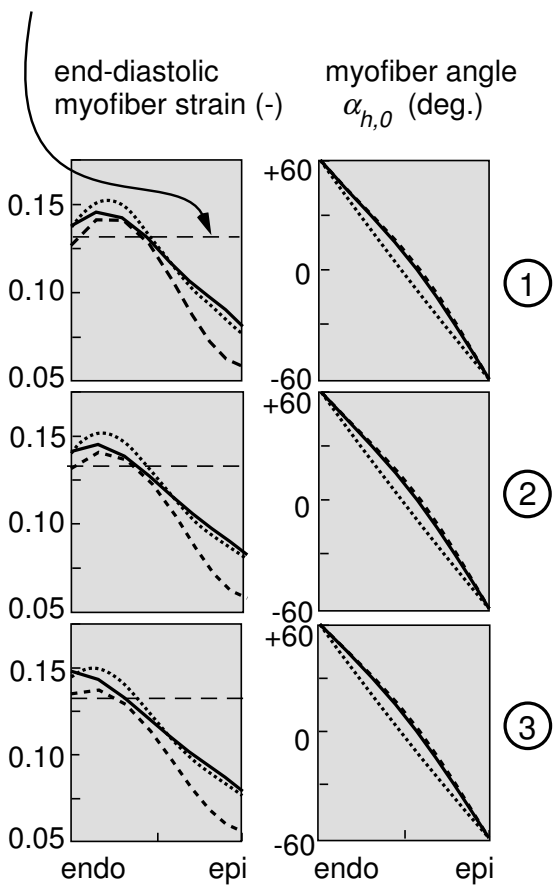

E

Figure 6.5. Transmural distribution of growth at three different levels from endocardium (endo) to epicardium (epi) as indicated in Fig 6.4A. A With both fixed and updated reference, similar volume changes are observed. B For both approaches, the observed change in cross-myofiber area is similar as well. C Differences are observed in subendocardial changes in unloaded myofiber length. Note the much larger amplitude of changes in cross-myofiber area as compared to changes in myofiber length. D Change in enddiastolic myofiber strain. When updating the reference configuration, end-diastolic strain becomes closer to the homeostatic value over the whole transmural range. When fixing the reference, end-diastolic strains from midwall to epicardium diverge from the homeostatic value. E Due to inhomogeneous volumetric growth, the transmural distribution of myofiber angles $\alpha_{h, 0}$ in the externally unloaded tissue changes from a linear to a more parabolic one. 
area and change in myofiber length, it becomes evident that volumetric growth predominantly occurs through a change in cross-myofiber area (Fig 6.5B and C). With both approaches, cross-myofiber area increases in the subendocardium and decreases in the subepicardium. Differences arise between both simulations, when regarding the change in myofiber length. With both approaches, subepicardial myofibers shorten. However, subendocardial myofibers shorten when using the updated reference approach, while they lengthen when using the fixed reference approach (Fig 6.5C).

Growth using an updated reference caused end-diastolic myofiber strains over the complete transmural range to become closer to the homeostatic values (Fig 6.5D). With a fixed reference, however, myofiber strains decreased over the whole transmural range, resulting in divergence from the homeostatic values in midwall and subepicardial regions.

As a consequence of the transmural inhomogeneity of growth, in both simulations the transmural distribution of the myofiber angles evolved from a linear to a more parabolic shape (Fig 6.5E). Regions with positive growth exhibited a decreased transmural gradient of fiber angle $\alpha_{h, 0}$ in the externally unloaded configuration, whereas an increase in transmural gradient was observed in regions with negative growth.

\subsection{DISCUSSION}

The aim of the present study was two-fold. The first aim was to develop and test a method to simulate load-induced inhomogeneous three-dimensional volumetric growth of a complex geometry such as the cardiac LV. Generally, our approach to simulate a load-induced increment in volumetric growth involves three steps; 1) simulation of beat-to-beat tissue mechanics to determine a load measure relevant for growth; deviation of this measure from its homeostatic value yields the growth stimulus; 2) the stimulus is related to a desired amount of volume change, which is subsequently translated into a growth tensor $\boldsymbol{F}_{g}$ that describes unconstrained tissue growth; and 3) based on the growth tensor, a simulation of growth mechanics is used to deform the unloaded tissue configuration to an intact locally grown tissue. Commonly a fixed reference is used to compute growth during multiple increments. However, in light of continuous turnover occurring together with growth, such a fixed reference is unlikely to exist in reality. Therefore, as a second aim we investigated the effect of updating the stress-free reference after each growth step on the grown geometry.

To illustrate our model we simulated LV growth in response to end-diastolic myofiber strain. With both fixed and updated reference approach, similar growth behavior could be observed, as far as changes in tissue volume, cross-myofiber area, and myofiber orientation are concerned (figure 6.5). With both approaches, 
volume changes in magnitude of $30 \%$ could be obtained. However, end-diastolic strain developed differently between both approaches. While growth with an updated reference caused end-diastolic myofiber strain across the wall to develop towards the homeostatic value, growth with a fixed reference resulted in midwall and subepicardial strains that were further apart from the homeostatic value. Apparently, our simulations have not progressed to a point where differences in enddiastolic strain between the two approaches clearly affect the observed volumetric and cross-sectional change. However, our results show that fixing or updating the reference configuration may eventually have a significant effect on the achieved growth.

It remains unclear which of the two approaches is more realistic. Fixing the reference configuration causes shape changes during growth to result in accumulation of internal stresses in the externally unloaded tissue. These residual stresses are usually assumed to cause the opening of the externally unloaded tissue upon incision. As such, comparison of model-predicted opening angles with experimentally determined ones has been used as a way to check the growth model (26). Although the traditionally used growth model with a fixed reference provides for a theoretical link between growth and residual stress development, unfortunately however, to the best of knowledge, no direct experimental evidence exists that either supports or rejects a causal relation between growth and residual stress build-up. In contrast, when considering tissue turnover, stresses due to growth are more likely to be related to incremental growth rather than to accumulated growth, i.e., turnover causes constituents bearing the residual stresses to be replaced. Therefore, it may be more physiological to update the stress-free reference configuration after each growth increment instead of fixing it. However, when updating the reference, build-up of residual stress during growth is limited to the last increment. To still account for the existence of residual stresses in real mature tissue we note that it has been hypothesized that, during turnover, new constituents may be added in the existing tissue under pre-stretch, thereby affecting the residual stress state of the total tissue $(12,29)$. As such, residual stresses may originate from a combination of G\&R.

Experimental data remain unclear about the relation between the growth stimulus and the response $(11,17)$. As a consequence, several assumptions had to be made in the growth model. First, we used end-diastolic myofiber strain as growth trigger, since in vitro stretching of myocytes has been shown to induce a hypertrophic response $(21,22)$. However, experimental data remain inconclusive and other candidates remain plausible. For instance, growth due to increased filling in vivo was also found to correlate to ejection (myofiber) strain and end-diastolic stress $(5,9)$. To be more specific, Donker et al. 2005 found a correlation between normalization of ejection strain and end-diastolic stress on the one hand, and a decrease in growth rate on the other. As an approximation, we adopted a linear relation between the growth rate and the stimulus (Eq 6.8). 
Second, we assumed unconstrained growth to be isotropic, since no experimental data exists about the directionality of unconstrained tissue growth. Experimental data shows that, depending on the loading conditions, the intact cardiac LV can exhibit eccentric growth, in which mainly length of the myofibers increases, or concentric growth, in which mainly the thickness of the myofibers increases (8). Interestingly, our simulations show that isotropic unconstrained growth yields an anisotropic pattern in the intact heart, as volume changes predominantly occurred by a change in myofiber cross-sectional area (figure Fig 6.5A through $\mathrm{C}$ ). Should experimental data about the directionality of growth become available, it can readily be incorporated by changing the form of growth tensor $\boldsymbol{F}_{g}(18,20)$.

Third, we assumed strain energy density functions during loading $\left(W_{l}\right)$ and growth $\left(W_{g}\right)$ to be the same. However, as stated by (12): "a constitutive relation does not define a material, it merely quantifies a behavior under certain circumstances". In other words, the short-term behavior of cardiac tissue on a beat-to-beat time scale may differ from the behavior on a time scale of growth, i.e., $W_{l} \neq W_{g}$.

Finally it is noted that, in our model, we implicitly included the effect of turnover by updating the reference. In the future, the growth model should be extended with more detailed description of turnover and remodeling, capable of including an explicit relation between (changes in) local tissue behavior (e.g., orientation, stiffness, pre-stretch etc.) and (changes in) local volume. The theory of mixtures as proposed by Humphrey and Rajagopal 2002 provides an elegant approach to do this. We envisage that such an extension enables the model to capture a much wider range of physiological processes, thereby potentially generating additional insights into the relation between local mechanical stimuli and G\&R.

\subsection{SUMMARY AND CONCLUSIONS}

A method has been presented to simulate load induced inhomogeneous volumetric growth in a complex tissue geometry and, furthermore, to asses the effect of fixing or updating of the stress-free reference configuration. In the cardiac left ventricle, inhomogeneous volume changes in magnitude of $30 \%$ could be simulated by using a deviation in end-diastolic myofiber strain from its homeostatic value as stimulus. As growth progressed and the reference configurations in the two approaches started to diverge, the end-diastolic strain also became different. With an updated reference, stimulus amplitude decreased over time everywhere in the wall. However, with a fixed reference no such reduction in stimulus was observed. It is concluded that, when modeling volumetric growth, the choice of the reference configuration is of great importance for the computed growth. 


\section{ACKNOWLEDGEMENTS}

This research was financially supported by grant 2007B203 of the Netherlands Heart Foundation.

\section{REFERENCES}

1. Alastrue V, Pena EA, Martinez MA, Doblare M (2007) Assessing the use of the "opening angle method" to enforce residual stresses in patient-specific arteries. Ann Biomed Eng 35:18211837.

2. Bathe KJ (1982) Finite element procedures in engineering analysis. Prentice-Hall Inc., Englewood Cliffs, New Jersey.

3. Bovendeerd PH, Arts T, Huyghe JM, van Campen DH, Reneman RS (1992) Dependence of local left ventricular wall mechancis on myocardial myofiber orientation: a model study. J Biomech 25:1129-1135.

4. Costa KD, Holmes JW, McCulloch AD (2001) Modeling cardiac mechanical properties in three dimensions. Phil Trans R Soc Lond A 359:1233-1250.

5. Donker DW, Volders PG, Arts T, Bekkers BC, Hofstra L, Spätjens RL, Beekman JD, Borgers M, Crijns HJ, Vos MA (2005) End-diastolic myofiber stress and ejection strain increase with ventricular volume overload: Serial in-vivo analyses in dogs with complete atrioventricular block. Basic Res Cardiol 100:372-382.

6. Emery JL, Omens JH (1997) Mechanical regulation of myocardial growth during volumeoverload hypertrophy in the rat. Am J Physiol Heart Circ Physiol 273:H1198-H1204.

7. Geerts-Ossevoort L (2003) Cardiac myofiber reorientation: a mechanism for adaptation? Ph.D. thesis Eindhoven University of Technology, Eindhoven, The Netherlands.

8. Gerdes AM, Capasso JM (1995) Structural remodeling and mechanical dysfunction of cardiac myocytes in heart failure. J Mol Cell Cardiol 27:849-856.

9. Holmes JW (2004) Candidate mechanical stimuli for hypertrophy during volume overload. J Appl Physiol 97:1453-1460.

10. Holmes JW, Borg TK, Covell JW (2005) Structure and mechanics of healing myocardial infarcts. Annu Rev Biomed Eng 7:223-253.

11. Humphrey JD (2001) Stress, strain and mechanotransduction in cells. J Biomech Eng 123:638641.

12. Humphrey JD, Rajagopal KR (2002) A constrained mixture model for growth and remodeling of soft tissues. Math Model Meth Appl Sci 12:407-430.

13. Kerckhoffs RC, Bovendeerd PH, Kotte JC, Prinzen FW, Smits K, Arts T (2003) Homogeneity of cardiac contraction despite physiological asynchrony of depolarization: a model study. Ann Biomed Eng 31:536-547.

14. Kuhl E, Maas R, Himpel G, Menzel A (2007) Computational modeling of arterial wall growth. attempts towards patient-specific simulations based on computer tomography. Biomechan Model Mechanobiol 6:321-331.

15. MacKenna D, Summerour SR, Villarreal FJ (2000) Role of mechanical factors in regulating fibroblast function and extracellular matrix synthesis. Cardiovasc Res 46:257-263.

16. Nash MP, Hunter PJ (2000) Computational mechanics of the heart: from tissue structure to ventricular function. J Elas 61:113-141.

17. Omens JH (1998) Stress and strain as regulators of myocardial growth. Prog Biophys Mol Biol 69:559-572.

18. Ramasubramanian A, Latacha KS, Benjamin JM, Voronov DA, Ravi A, Taber LA (2006) Computational model for early cardiac looping. Ann Biomed Eng 34:1655-1669. 


\section{Modeling volumetric growth}

19. Rodriguez EK, Hoger A, McCulloch AD (1994) Stress-dependent finite growth in soft elastic tissues. J Biomech 27:455-467.

20. Rodriguez J, Giocolea JM, Gabaldon F (2007) A volumetric model for growth of arterial walls with arbitrary geometry and loads. J Biomech 40:961-971.

21. Sadoshima J, Izumo S (1997) The cellular and molecular response of cardiac myocytes to mechanical stress. Ann Rev Physiol 59:551-571.

22. Simpson DG, Majeski M, Borg TK, Terracio L (1999) Regulation of cardiac myocyte protein turnover and myofibrillar structure in vitro by specific directions of stretch. Circ Res 85:e59e69.

23. Skalak E, Dasgupta G, Moss M, Otten E, Dullemeijer P, Vilmann H (1982) Analytical description of growth. J Theor Biol 94:555-577.

24. Stevens C, Remme E, LeGrice IJ, Hunter P (2003) Ventricular mechanics in diastole: material parameter sensitivity. J Biomech 36:737-748.

25. Taber LA (1998) Mechanical aspects of cardiac development. Prog Biophys Mol Biol 69:237255.

26. Taber LA, Humphrey JD (2001) Stress-modulated growth, residual stress, and vascular heterogeneity. J Biomech Eng 123:528-535.

27. Usyk TP, LeGrice IJ, McCulloch AD (2002) Computational model of three-dimensional cardiac electromechanics. Comp Visual Sci 4:249-257.

28. van Oosterhout MF, Prinzen FW, Arts T, Schreuder JJ, Vanagt WY, Cleutjens JP, Reneman RS (1998) Asynchronous electrical activation induces asymmetrical hypertrophy of the left ventricular wall. Circ 98:588-595.

29. Watton PN, Hill NA, Heil M (2004) A mathematical model for the growth of the abdominal aortic aneurysm. Biomechan Model Mechanobiol 3:98-113. 



\section{General discussion}




\subsection{INTRODUCTORY REMARKS}

Mechanically induced growth and remodeling $(G \& R)$ play an important role in long-term adaptation of cardiac pump capacity to match the needs of the body during health and disease. Experimental approaches have contributed significantly to our understanding of mechanically induced G\&R in the heart. However, the quantitative relation between the mechanical trigger (e.g., stress or strain), the evoked tissue response, and the resulting change in cardiac pump capacity is not completely understood. This is mainly due to limitations of the experimental methodology.

The aim of this thesis was to gain additional insights in stimulus-response relations in cardiac G\&R using mathematical models. These mathematical models include 1) a model of cardiac mechanics that quantitatively describes the forward relation between local tissue properties and organ function, and 2) adaptation rules that account for the mechanical feedback resulting in changes in tissue properties. We specifically focussed on volumetric growth and myofiber reorientation. The mathematical models were evaluated by comparison of predicted geometry, myofiber orientations, and deformation with experimental data on the healthy left ventricle (LV).

In this chapter, we will give an overview of the studies presented in this thesis and subsequently put them into a broader perspective.

\subsection{MYOFIBER ORIENTATIONS AND TORSION IN THE NOR- MAL AND SITUS INVERSUS TOTALIS LEFT VENTRICLE}

Transmural variation in LV myofiber orientation is reflected in the apex-to-base gradient in rotation (=torsion). Therefore, we measured torsion with magnetic resonance tagging (MRT) (chapter 2). In a normal healthy control group we found that LV torsion at end of ejection is about equally negative at all longitudinal levels, suggesting that the transmural variation in myofiber orientation is similar at all longitudinal levels. This is supported by data from histology $(19,33)$ and magnetic resonance diffusion tensor imaging (MRDTI) experiments (16).

We also measured LV torsion in case of complete left-right mirroring of internal organ anatomy and position (situs inversus totalis, SIT). In contrast to the normal control group, LV torsion in the SIT group, on average, was found to exhibit a change in sign from the apex to the base; basal torsion was opposite in sign with respect to that in normal LV base, but apical torsion in the SIT LV was similar to that in the normal LV. These results strongly suggest that the transmural variation in myofiber orientations in SIT near the base is mirrored with respect to normal situation, but that the myofiber orientations near the apex are normal. Indeed, limited histological data confirms mirroring of the basal myofiber orientations with 
respect to normal $(4,28)$. Thus, the SIT LV structure is substantially different from the structure in the normal LV.

Subsequently, we investigated whether the stimulus-response relations that could predict a realistic LV structure and deformation for the normal heart, can also predict a realistic structure and deformation for the SIT heart (chapter 3). Based on experimental observations, we hypothesized that 1) myofiber mass increases in response to local overstretching at the beginning of ejection and to an elevated global contractility, 2) cavity volume increases in response to large strain excursions, and 3) myofibers reorient to homogenize myofiber strain during ejection and to correct their misalignment with neighboring myofibers (3). The hypotheses were tested using a relatively simple model of cardiac mechanics in which the LV was represented by a stack of nested cylindrical shells. After G\&R, a stable structure was found in which the myofiber orientations qualitatively agreed with the limited histological data available in SIT. In addition, LV torsion in SIT was found to exhibit the same reversal as found in the tagging experiments, while predicted cavity volume and wall mass were realistic as well.

\subsection{THE ROLE OF SHEAR DEFORMATION IN 3D MYOFIBER REORIENTATION}

Although the results obtained with the shell model substantiate a physiological relevance for the hypothesized stimulus-response relations, the shell model had several limitations, i.e., 1) geometry was restricted to a cylinder, 2) deformation was restricted to a combination of ejection, axial stretch, and torsion, and 3) myofibers were assumed to be orientated parallel to the cavity, thus lacking a transmural component in orientation. Furthermore, myofiber reorientation was implemented by simply penalizing misalignment and deviations of myofiber strain from a set homeostatic value. In this optimization we did not explicitly model a causal relation between a mechanical stimulus and myofiber reorientation.

To alleviate limitations of the shell model and investigate a more causal relation between strain, misalignment, and myofiber reorientation in three dimensions (including adaptation of the transmural component in orientation), we subsequently used a more versatile three-dimensional finite element (FE) model (chapter 4). As a hypothetical causal relation, we reasoned that misaligned myofibers would straighten upon contraction, leading to fiber cross-myofiber shear that locally disrupts connections between the extra-cellular matrix (ECM) and the myofibers. Eventually, turnover of the ECM and/or renewal of the connections between the ECM and the myofibers would cause the myofiber orientation to rotate towards the (shear) deformed myofiber orientation. We started our simulation with a structure in which, similar to the shell model, myofiber orientations had no transmural component. After adaptation of the myofiber orientations to shear, a myofiber 
orientation pattern developed in which transmural components were within the range of those observed experimentally with MRDTI. Due to this transmural component, spatially averaged peak myofiber stress and myofiber strain during ejection increased. Furthermore, the LV was able to generate more pressure and flow, even though tissue mass in the model did not change. Simultaneously, we found that myofiber stress and myofiber strain became more homogeneously distributed throughout the cardiac wall. These results suggest an important role for fiber cross-fiber shear deformation in the genesis of cardiac structure and homogenization of mechanical load. Furthermore, our results substantiate the finding that the (spatial distribution of the) transmural component in myofiber orientation is an important determinant for local tissue mechanics as well as for global pump capacity $(9,35)$.

While it is promising that the predicted myofiber orientations are within the range of experimental data, it is noted that this agreement is not a very critical test. Myofiber orientations are subject to an inaccuracy of about $\pm 6^{\circ}$, even when measured with the gold standard of MRDTI (16). However, due to a high sensitivity of shear deformation to myofiber orientations $(9,35)$, a comparison of shear strains between model and experiment offers an additional, more critical test. In this context, we compared torsion and circumferential-radial shear strain in the model before and after shear-induced myofiber reorientation with values determined from magnetic resonance tagging (MRT) experiments (chapter 5). We found that after adaptation the model predictions in shear strains were much closer to the experimental data. Nevertheless, discrepancies remained.

\subsection{MODELING 3D INHOMOGENEOUS VOLUMETRIC GROWTH}

Discrepancies between the model and the experimental data are likely to be the result of simplifications introduced in the model. Perhaps the most crude simplification is that we only adapted the myofiber orientations, i.e., all other model parameters such as stiffness, unloaded sarcomere length, and unloaded geometry remained unchanged during the finite element simulations.

In the shell model (chapter 3) we found that growth and myofiber reorientation are sufficient to yield a stable structure and geometry. Therefore, we subsequently focussed on simulating three-dimensional, inhomogeneous volumetric growth with the FE model (chapter 6). Using end-diastolic myofiber strain as stimulus for (isotropic) volumetric growth, we could successfully simulate significant inhomogeneous growth with volume changes in magnitude of $30 \%$. It was found that in simulating volumetric growth with an FE model, the choice of the reference configuration is of great importance for the computed growth. Usually the initial configuration is used as reference throughout the entire growth process $(1,2,26,29)$. Following the same approach we found that growth led to a more 
heterogeneous end-diastolic strain distribution, i.e., not leading to normalization of the mechanical load. However, in case the reference was updated after each growth step, growth led to homogenization of end-diastolic strain. This suggest the latter approach to be more realistic. However, it should be mentioned that, with volumetric growth alone, no steady-state could be reached.

\subsection{STUDY LIMITATIONS AND FUTURE PERSPECTIVES}

\subsubsection{Mathematical models and numerical considerations}

In this thesis we used mathematical models to investigate cardiac G\&R. Construction of such a mathematical model generally involves three parts, i.e., 1) balance equations with appropriate boundary conditions are specified, 2) constitutive laws are formulated to describe the behavior of the tissue, and 3) numerical methods are designed to efficiently solve the balance equations. Usually, a compromise has to be found between versatility in application of the model and computational demand.

In this study we used two of the most commonly applied types of cardiac mechanics models; a shell model (chapter 3) and a finite element model (chapters 4-6). The advantage of a shell model is that the computational demand is low and that numerical implementation is fairly simple $(3,8,34)$. As such, they allow for quick assessment of hypothesized adaptation mechanisms (e.g., myofiber reorientation or growth). However, their applications are confined to situations with a simple LV geometry in which the number of constitutive properties and their spatial variation are restricted. Three-dimensional FE based models are much more versatile, not restricted to any specific type of inhomogeneity in tissue properties or geometry. However, numerical implementation is quite difficult. In particular, the implementation of three-dimensional (3D) inhomogeneous volumetric growth is challenging. This problem was successfully solved in chapter 6. However, a disadvantage of a 3D FE model is that computational demand is quite high (23). Even though in the last four years about a 60 -fold reduction in computation time could be achieved due to faster computers and improved numerical algorithms $(22,25)$, a typical simulation of myofiber reorientation over 40 cardiac cycles still needs about a week to compute on a standard PC.

To investigate the effect of assumptions in material parameters such as passive stiffness, a parameter sensitivity analysis is required. However, such an analysis in a 3D FE model is severely hampered by the high computational demand. For future studies, computational demand may be significantly reduced by explicitly assuming rotational symmetry in the numerical formulation of the FE model (7, 27). Unfortunately, such a numerical formulation does not yet exist in the used finite element package (SEPRA, Leidschendam, The Netherlands). 
It should be mentioned that in our studies we primarily focussed on local cardiac mechanics. In the shell model, systemic blood pressure was essentially prescribed, whereas in the FE model global hemodynamics was accounted for by a simple cavity pressure and a windkessel model. Future studies may be aimed at including a more realistic model of global hemodynamics that, for instance, also includes blood pressure regulation.

\subsubsection{Combination of multiple adaptation mechanisms}

In the FE model we found that through myofiber reorientation alone we could not fully reproduce the deformation patterns in the LV. Furthermore, with volumetric growth alone we could not arrive at a steady state solution. In the shell model, however, we found that a combination of growth and myofiber reorientation is sufficient to yield a stable LV geometry and structure. Therefore, the logical next step in FE modeling is to combine volumetric growth and myofiber reorientation.

In reality, besides tissue mass and pattern of myofiber orientations, it is likely that more tissue properties are regulated through mechanical load. For instance, Williams and Goldspink 1978 found evidence of sarcomere length adaptation in immobilized skeletal muscles. They found that in case immobilization of the muscle occurred in the extended state, sarcomeres were added in order to reduce the length of the remaining sarcomeres. In contrast, when the muscle was fixed in the shortened state, the number of sarcomeres decreased, leading to an increase in sarcomere length. They found that the remaining sarcomere length was such that maximum tension was developed in the immobilized position. Apparently, sarcomere length in skeletal muscle is adapted so that its function (force generation) is optimized. Translated to the cardiac muscle, it could be hypothesized that sarcomere length is adapted to maximize contraction force during ejection. Rodriguez et al. 1993 found that in the unloaded LV (approximately the situation during early filling), sarcomere length increased from about $1.76 \mu \mathrm{m}$ at the endocardium to about $1.91 \mu \mathrm{m}$ at the epicardium. This transmural difference is reduced during filling, leading to a homogeneously distributed sarcomere length of about $2.1 \mu \mathrm{m}$ at the start of contraction. This indeed is the sarcomere length were isometrically developed force is about maximal $(11,21)$. The effects of sarcomere length control can readily be investigated with our FE model, particularly because inclusion of sarcomere control in the FE model requires little effort, e.g., by setting the sarcomere length at the start of contraction at a specified value.

In addition, stiffness may change due to an altered tissue composition. For instance, in case of volume overload, growth is accompanied with stiffening of the myocardium due to increased collagen content (14). Change in stiffness is readily included through a parameter change in the constitutive model. 


\subsubsection{Constitutive modeling: tissue as a mixture of compo- nents}

The interaction between the passive ECM and the active myofibers was hypothesized to play an important role in the regulation of myofiber orientation through fiber cross-fiber shear strain (chapter 4). Our successful attempt in producing stable and realistic myofiber orientations suggests that the interaction between the ECM and myofibers plays an important role in development and maintenance of a stable cardiac structure.

Baicu et al. 2003 showed that the ECM plays an important role in transducing myocyte contractions into myocardial force development. Disruption of the ECM in isolated papillary muscles significantly limited the muscle's ability to generate force and to shorten, even though myocyte contractile performance remained the same. Conversely, van Bavel et al. 2006 showed that muscle contractions are essential in ECM maintenance. In a blood vessel (arteriole) they found that an increased muscle-tone leads to a significant reduction in vessel diameter. To explain this observation they formulated the tone-remodeling hypothesis in which muscle contraction basically determines ECM dimensions (Fig 7.1). They postulated that smaller blood vessel dimensions due to increased tone are enforced by cross-links between the existing ECM and a new generation of ECM constituents (e.g., newly synthesized collagen). Applied to the heart, myofiber contraction may be required to maintain ECM dimensions and to protect the heart from dilation. Alterations in ECM-myofiber interaction, including integrin turnover (18), may thus play a vital role during cardiac disease. However, much is unclear about the interaction between the ECM, the fibroblasts responsible for secretion of ECM constituents, and the myocytes.

The tone-remodeling concept by van Bavel et al. 2006 is similar to the concept of evolving natural configurations by Humphrey and Rajagopal 2002. Both concepts state that adaptation involves interaction between different tissue constituents of several generations. Furthermore, each generation of tissue constituents is considered to have its own natural configuration (e.g., stiffness, orientation, unloaded length) that may evolve through time.

The fact that the life-time of the tissue constituents is relatively short (from seconds to weeks (31)) implies that tissue has limited 'memory'. In our growth simulation, the latter concept was implicitly included by updating the unloaded reference configuration during growth (chapter 6). The tone-remodeling hypothesis also implies that in the externally unloaded tissue not all tissue components are at their unloaded configuration, i.e., some components are stretched while others are shortened. This may explain why residual stresses are found in intact cardiovascular tissues $(10,15,30)$.

Explicit inclusion of different tissue constituents, with different natural configurations is not possible in the current constitutive model. We quantified the (passive) 
mechanical behavior of the tissue by a strain energy density function with respect to an unstressed geometry. This function contains parameters that do not relate directly to measurable parameters of tissue composition such as collagen content (32). To overcome this problem, a (constrained) mixture approach could be considered in which the tissue is explicitly modeled as a mixture of constituents that each have their own natural configurations subject to their own mechanically regulated turnover (20). An additional advantage is that in this way experimentally measurable quantities such as collagen content can be directly included into the model. Constrained mixture models are now widely adopted in the investigation of vascular $G \& R(2,5,13,17)$. However, for cardiac tissue such a model does not yet exist. Future studies may be aimed at the development of a constrained mixture model for cardiac tissue.

\subsubsection{Mathematical models as a diagnostic tool}

Our simulations show that mechanical load as generated by the heart itself is a sufficient trigger for the development and maintenance of cardiac geometry and structure, i.e., the myocardium appears self-structuring. This observation may be used in the development of patient specific models, which for instance could be used in selection of disease treatment or be used to noninvasively determine parameters of cardiac function such as local tissue stresses or strains $(12,24)$.

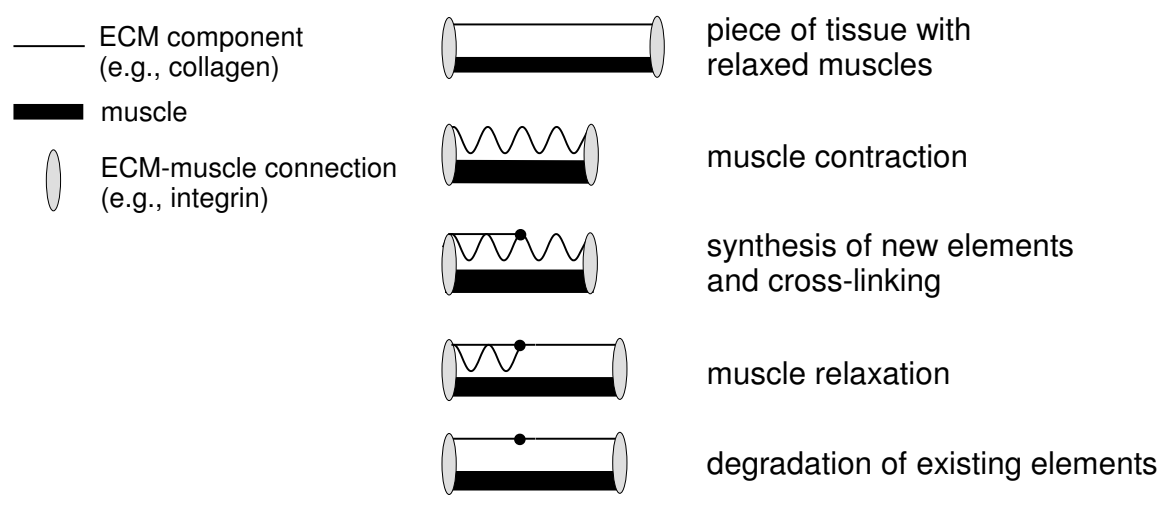

Figure 7.1. Tone-remodeling hypothesis by van Bavel et al. 2006. In the reference situation, the blood vessel is inflated and the extra-cellular matrix is loaded. Contraction of the muscle unloads the extra-cellular matrix (ECM). New ECM components are synthesized and cross-linked to the existing ECM, which due to muscle contraction now has a smaller dimension. Following muscle relaxation, the ECM will no longer extend to its original length. Eventually, older unloaded generations of ECM constituents will be removed from the tissue. 
Usually the model parameters are fitted to experimental data. However, such an approach may be limited by inaccuracies or impossibility of in vivo measurements. Consequently, the number of measurements may be insufficient to uniquely determine the parameters. Perhaps, adaptation rules could be used to estimate these parameters instead. Especially, in view of inaccuracies in MRDTI, adaptation rules may provide a more accurate estimation of the myofiber orientations. In this way the number of measurements required to determine a patient specific set of parameters can be greatly reduced.

\subsection{GENERAL CONCLUSIONS}

In this thesis we developed and applied mathematical models to investigate cardiac growth and remodeling $(G \& R)$. In a simple shell model we found that local mechanical regulation of tissue growth and myofiber reorientation was sufficient to predict a realistic geometry and structure. This finding suggests a primary role for mechanical load in myocardial development and maintenance of myocardial structure. Our finite element simulations furthermore indicate that myofiber cross-myofiber shear deformation may be one of the main mechanical load stimuli through which myofiber reorientation occurs. In addition, our simulations indicate that the transmural component in myofiber orientation influences the pump capacity of the heart significantly. Thus, myofiber reorientation was identified as a highly potent mechanism for control of local mechanical load as well as global pump capacity. In a finite element model, myofiber reorientation alone was found to be insufficient to fully reproduce the experimentally observed shear strains. In agreement with our findings in the shell model, this may possibly be due to absence of other adaptive mechanisms such as volumetric growth. Therefore, we also developed a method to simulate three-dimensional volumetric growth. Future combinations of myofiber reorientation with volumetric growth and/or other G\&R responses may help to gain additional quantitative insights in the relation between changes in mechanical load, changes in tissue geometry and structure, and changes in pump capacity.

\section{REFERENCES}

1. Alastrue V, Pena EA, Martinez MA, Doblare M (2007) Assessing the use of the "opening angle method" to enforce residual stresses in patient-specific arteries. Ann Biomed Eng 35:18211837.

2. Alford PW, Humphrey JD, Taber LA (2007) Growth and remodeling in a thick-walled artery model: effects of spatial variations in wall constituents. Biomechan Model Mechanobiol 7:245262.

3. Arts T, Prinzen FW, Snoeckx LH, Rijcken JM, Reneman RS (1994) Adaptation of cardiac structure by mechanical feedback in the environment of the cell: a model study. Biophys J 66:953961. 


\section{Chapter 7}

4. Asami I, Koizumi K (1989) [the vortex cordis is never reversely directed, even in situs inversus and L-loop anomaly] Kaibogaku Zasshi 64:36-45.

5. Baek S, Rajagopal KR, Humphrey JD (2006) A theoretical model of enlarging intracranial fusiform aneurysms. J Biomech Eng 128:142-149.

6. Baicu CF, Stroud JD, Livesay VA, Hapke E, Holder J, Spinale FG, Zile MR (2003) Changes in extracellular collagen matrix alter myocardial systolic performance. Am J Physiol Heart Circ Physiol 284:H122-H132.

7. Bathe KJ (1982) Finite element procedures in engineering analysis. Prentice-Hall Inc., Englewood Cliffs, New Jersey.

8. Beyar R, Sideman S (1984) A computer study of the left ventricular performance based on fiber structure, sarcomere dynamics, and transmural electrical propagation velocity. Circ Res 55:358-375.

9. Bovendeerd PH, Huyghe JM, Arts T, van Campen DH, Reneman RS (1994) Influence of endocardial-epicardial crossover of muscle fibers on left ventricular wall mechanics. J Biomech 27:941-951.

10. Costa KD, May-Newman K, Farr D, O'Dell WG, McCulloch AD, Omens JH (1997) Threedimensional residual strain in midanterior canine left ventricle. Am J Physiol Heart Circ Physiol 273:H1968-H1976.

11. de Tombe PP, ter Keurs HE (1991) Sarcomere dynamics in cat trabeculae. Circ Res 68:588596.

12. Donker DW, Volders PG, Arts T, Bekkers BC, Hofstra L, Spätjens RL, Beekman JD, Borgers M, Crijns HJ, Vos MA (2005) End-diastolic myofiber stress and ejection strain increase with ventricular volume overload: Serial in-vivo analyses in dogs with complete atrioventricular block. Basic Res Cardiol 100:372-382.

13. Driessen NJ, Cox MA, Bouten CV, Baaijens FP (2008) Remodelling of the angular collagen fiber distribution in cardiovascular tissues. Biomech Model Mechanobiol 7(2):93-103.

14. Emery JL, Omens JH (1997) Mechanical regulation of myocardial growth during volumeoverload hypertrophy in the rat. Am J Physiol Heart Circ Physiol 273:H1198-H1204.

15. Fung YC (1991) What are the residual stresses doing in our blood vessels? Ann Biomed Eng 19:237-249.

16. Geerts L, Bovendeerd P, Nicolay K, Arts T (2002) Characterization of the normal cardiac myofiber field in goat measured with MR-diffusion tensor imaging. Am J Physiol Heart Circ Physiol 283:H139-H145.

17. Gleason RL Jr, Humphrey JD (2005) A 2D constrained mixture model for arterial adaptations to large changes in flow, pressure and axial stretch. Math Med Biol 22:347-369.

18. Goldsmith EC, Carver W, McFadden A, Goldsmith JG, Price RL, Sussman M, Lorell BH, Cooper G, Borg TK (2003) Integrin shedding as a mechanism of cellular adaptation during cardiac growth. Am J Physiol Heart Circ Physiol 284:H2227-H2234.

19. Greenbaum RA, Ho SY, Gibson DG, Becker AE, Anderson RH (1981) Left ventricular fibre architecture in man. Brit Heart J 45:248-263.

20. Humphrey JD, Rajagopal KR (2002) A constrained mixture model for growth and remodeling of soft tissues. Math Model Meth Appl Sci 12:407-430.

21. Kentish JC, ter Keurs HE, Ricciardi L, Bucx JJ, Noble MI (1986) Comparison between the sarcomere length-force relations of intact and skinned trabeculae from rat right ventricle. Influence of calcium concentrations on these relations. Circ Res 58:755-768.

22. Kerckhoffs RC, Bovendeerd PH, Kotte JC, Prinzen FW, Smits K, Arts T (2003) Homogeneity of cardiac contraction despite physiological asynchrony of depolarization: a model study. Ann Biomed Eng 31:536-547.

23. Kerckhoffs RC, Healy SN, Usyl TP, McCulloch AD (2006) Computational methods for cardiac electromechanics. Proc IEEE 94:769-783. 
24. Kerckhoffs RCP, Lumens JE, Gu Q, Bassingthwaighte JB, Omens JH, McCulloch AD (2008) Cardiac resynchronization: Insight from experimental and computational models. Prog Biophys Mol Biol 97:543-561.

25. Kroon W, Delhaas T, Bovendeerd P, Arts T (2008) Computational analysis of the myocardial structure: Adaptation of cardiac myofiber orientations through deformation. Med Imag Anal doi:10.1016/j.media.2008.06.015.

26. Kuhl E, Maas R, Himpel G, Menzel A (2007) Computational modeling of arterial wall growth. attempts towards patient-specific simulations based on computer tomography. Biomechan Model Mechanobiol 6:321-331.

27. Malvern LE (1969) Introduction to the Mechanics of a Continuous Medium. Prentice-Hall Inc., Englewood Cliffs, New Jersey.

28. Matsumura H, Aizawa $\mathrm{Y}$, Kumaki K (1990) Myocardial architecture in situs inversus viscerum totalis. In: Clark EB, Takao A (Eds) Developmental cardiology: morphogenesis and function, Futura Pub Co, Mount Kisco, pp. 605-624 (ISBN-13: 978-0-879-93382-1).

29. Rodriguez EK, Hoger A, McCulloch AD (1994) Stress-dependent finite growth in soft elastic tissues. J Biomech 27:455-467.

30. Rodriguez EK, Omens JH, Waldman LW, McCulloch AD (1993) Effect of residual stress on transmural sarcomere length distributions in rat left ventricle. Am J Physiol Heart Circ Physiol 264:H1048-H1056.

31. Russell B, Motlach D, Ashley WW (2000) Form follows function: how muscle shape is regulated by work. J Appl Physiol 88:1127-1132.

32. Schmid H, O'Callaghan P, Nash MP, Lin W, LeGrice IJ, Smaill BH, Young AA, Hunter PJ (2008) Myocardial material parameter estimation: a nonhomogeneous finite element study from simple shear tests. Biomechan Model Mechanobiol 7:161-173.

33. Streeter DD Jr, Spotnitz HM, Platel DJ, Ross J, Sonnenblick EH (1969) Fiber orientation in the canine left ventricle during diastole and systole. Circ Res 24:339-347.

34. Taber LA, Podszus WW (1997) A laminated shell model for the infarcted left ventricle. Int J Solids Struct 34:223-241.

35. Ubbink SW, Bovendeerd PH, Delhaas T, Arts T, van de Vosse FN (2006) Towards model based analysis of cardiac MR tagging data: relation between left ventricular shear strain and myofiber orientation. Med Imag Anal 10:632-641.

36. van Bavel E, Bakker EN, Pistea A, Sorop O, Spaan JA (2006) Mechanics of microvascular remodeling. Clinic Hemorheol Microcirc 34:35-41.

37. Williams PE, Goldspink G (1978) Changes in sarcomere length and physiological properties in immobilized muscle. J Anat 127:459-468. 



\section{Summary}


Normally, cardiac tissue develops in such a way that the heart is well able to deliver the pump capacity required by the body. Due to disease, however, pump capacity may diminish. Initially, growth (change in tissue mass) and remodeling (change in tissue properties such as orientation of the muscle fibers) will adapt the tissue to restore pump capacity, but as disease progresses, growth and remodeling may no longer be able to compensate.

Experimental observations have revealed mechanical tissue load to be an important regulator of cardiac tissue growth and remodeling. However, details of the mechanical stimuli and their related responses remain unclear, mainly due to limitations in the experimental methods. Additional knowledge of mechanically induced tissue growth and remodeling is needed to improve our understanding of cardiac development as well as to predict the long-term outcome of disease and the effect of possible treatment strategies.

In this thesis, we developed and applied mathematical models to gain more insight in the relation between local tissue load, local growth and remodeling of the tissue, and global pump capacity of the heart. Since mechanical load of the cardiac tissue is for a major part determined by the orientations and the mass of the muscle fibers (myofibers) in the wall, we focussed our modeling on myofiber growth and reorientation.

Direct assessment of cardiac myofiber orientations can currently only be done ex vivo. The pattern of myofiber orientations, however, is reflected in the deformation of the wall. In particular, the longitudinal gradient in rotation (rotation gradient in apex-base direction; torsion) is strongly dependent on the transmural course in myofiber orientations. Due to this dependency of wall deformation on the myofiber orientations in the wall, measurement of deformation indirectly provides additional information about the pattern of myofiber orientations. In chapter 2 we present an experimental study in which magnetic resonance tagging (MRT) was used to noninvasively and in vivo assess LV torsion at several longitudinal levels. In a normal control group we found that LV torsion at end of ejection is about equally negative at all measured longitudinal levels, indicating that the transmural course in myofiber orientation is similar at these levels. We also measured LV torsion in case of complete left-right mirroring of internal organ anatomy and position (situs inversus totalis, SIT). In contrast to the normal group, LV torsion in the SIT group, on average, was found to exhibit a change in sign from apex to base; basal torsion in the SIT LV was opposite in sign with respect to that in the normal LV, but apical torsion in the SIT LV was similar to that in the normal one. These results strongly suggest that, in accordance with the global mirroring of the anatomy, the transmural course in myofiber orientations in the SIT LV near the base is mirrored with respect to normal situation. However, near the apex, myofiber orientations in the SIT LV are normal.

In chapter 3, we describe a study on the stimulus-response relations for LV myofiber growth and reorientation. In a relatively simple two-dimensional (2D) 
shell model, these relations were investigated on their ability to reproduce cavity and wall volumes, as well as to reproduce patterns of myofiber orientations and torsion in the normal and in the SIT LV. Based on experimental findings from literature we hypothesized 1) that in case myofibers are overstretched at begin of ejection or in case myofibers have an elevated contractility, mass of the myofibers will increase to reduce the stretch and contractility, 2) that when myofiber strains during the cardiac cycle are large, the cavity will dilate to reduce the strain, and 3 ) that myofibers reorient to normalize their strain during ejection and to minimize the difference in orientation with respect to their neighbors. Subsequently, we simulated growth and myofiber reorientation in both the normal and the SIT LV, starting with unrealistic cavity and wall volumes, and an unphysiological pattern of myofiber orientations. In the model of the normal LV, realistic myofiber orientations, torsion, cavity volume and wall volume developed after growth and remodeling. For the SIT LV also a stable structure was found. The myofiber orientations qualitatively agreed with the limited histological data available in SIT, LV torsion was found to exhibit the same reversal as in the tagging experiments (chapter 2), and estimated cavity volume and wall volume were realistic and equal to those estimated in the normal LV. As such it was possible to reproduce experimental observations on myofiber orientations, torsion, and cavity and wall volumes for the normal as well as for the SIT LV on the basis of mechanical induced myofiber growth and reorientation.

In chapter 4 we used a more versatile three-dimensional (3D) finite element (FE) model to investigate a more detailed hypothesis regarding myofiber reorientation. We hypothesized that myofiber orientations are adapted so that their shear deformation is minimized. In the model simulation we started our simulation with a structure in which, similar to the 2D model, myofiber orientations had no transmural component (no transmural cross-over). After adaptation of the myofiber orientations to myofiber cross-myofiber shear, a myofiber orientation pattern developed in which transmural components were within the range of those observed experimentally with magnetic resonance diffusion tensor imaging (MRDTI). Due to this transmural component, spatially averaged peak myofiber stress and myofiber strain during ejection increased. Furthermore, the LV was able to generate more pressure and to eject more volume, even though tissue mass in the model did not change. Simultaneously, we found that myofiber stress and myofiber strain became more homogeneously distributed throughout the cardiac wall. These results suggest an important role for myofiber cross-myofiber shear deformation in the genesis of cardiac structure and homogenization of myofiber stress and strain. Furthermore, our results substantiate the finding that the (spatial distribution of the) transmural component in myofiber orientation is an important determinant for local tissue load as well as for global pump capacity.

It is promising that the predicted myofiber orientations are within the range of experimental data. Unfortunately, experimentally determined myofiber orienta- 
tions are subject to an inaccuracy of about $\pm 6^{\circ}$, even when measured with state of the art MRDTI techniques. As such, a comparison between model-predicted myofiber orientations and experimentally determined ones is not a very critical test for checking the model results. Due to the strong dependency of local longitudinal and transmural gradients in rotation on the myofiber orientation, a more critical test for the model is a comparison with experimental values for these gradients in rotation. Therefore, we compared longitudinal and transmural gradients in rotation in the model before and after shear-induced myofiber reorientation with values determined with MRT experiments (chapter 5). We found that after adaptation the model predictions were much closer to the experimental data. As such, our hypothesis on myofiber reorientation not only resulted in realistic (transmural components in) myofiber orientations, but also led to more realistic longitudinal and transmural gradients in rotation. Nevertheless, discrepancies remained between model and experiment.

In the simpler 2D model (chapter 3) we found that combined myofiber growth and reorientation are sufficient to yield a stable structure and geometry. In the 3D FE model simulations (chapters $\mathbf{4}$ and 5), we did not include growth. Therefore, we subsequently focussed on simulating inhomogeneous growth with the 3D FE model. FE models require a stress-free configuration to refer deformation and stresses against. Usually a single stress-free configuration is assumed as reference throughout the entire growth process. We reasoned that tissue turnover would change this reference configuration, and, hence, that updating the stressfree configuration over time might be more realistic. In chapter 6 , we studied the effect of fixing or updating the stress-free reference on the simulated growth. As a test case we simulated LV growth as induced by deviation of end-diastolic myofiber strain from a homeostatic value. With both approaches, we could successfully simulate significant inhomogeneous LV growth with volume changes of $30 \%$. We found that a fixed reference led to a more heterogeneous end-diastolic strain distribution. However, in case the reference was updated after each growth step, growth led to homogenization of end-diastolic strain. Since in reality a relatively homogeneous strain is observed, the latter approach might be more realistic. However, it should be mentioned that with volumetric growth alone, no steady-state could be reached in which the end-diastolic myofiber strains where at the homeostatic value.

In conclusion, we developed and applied mathematical models to investigate growth and remodeling of cardiac tissue. In a simple 2D shell model we found that local mechanical regulation of tissue growth and myofiber reorientation was sufficient to predict a realistic geometry and structure, both for the normal and the SIT LV. This result suggests a primary role for mechanical load in myocardial development and maintenance of myocardial structure. Our 3D finite element simulations furthermore indicate that myofiber cross-myofiber shear deformation may be one of the main mechanical load stimuli through which myofiber reorien- 
tation occurs. In addition, our simulations indicate that the transmural component in myofiber orientation influences the pump capacity of the heart significantly.

In the 3D model, myofiber reorientation alone was found to be insufficient to fully reproduce the experimentally observed longitudinal and transmural gradients in rotation. In agreement with our findings in the $2 \mathrm{D}$ shell model, this may possibly be due to absence of other adaptive mechanisms such as growth. Therefore, we also developed a method to simulate three-dimensional volumetric growth. Future combinations of myofiber reorientation, volumetric growth and/or other remodeling mechanisms may help to gain additional (quantitative) insights in the relation between changes in mechanical load, changes in tissue geometry and structure, and changes in pump capacity. 



\section{Samenvatting}


Normaal gesproken ontwikkelt het hartspierweefsel zich dusdanig dat het hart als geheel ruim in staat is de door het lichaam gevraagde pompcapaciteit te leveren. Echter, in het geval van een ziekte kan de pompcapaciteit van het hart achteruitgaan. Aanvankelijk zal door groei (verandering van weefselmassa) en remodellering (verandering van weefseleigenschappen zoals bijvoorbeeld de richting van de spiervezels) het weefsel proberen de pompcapaciteit te herstellen. Op de lange termijn kan het echter zijn dat groei en remodellering niet langer voldoende zijn om de pompcapaciteit te handhaven.

Experimentele studies hebben aangetoond dat de mechanische belasting van het weefsel als stimulus een grote rol speelt bij groei en remodellering. Details betreffende de specifieke stimulus en de bijbehorende reactie van het weefsel zijn echter nog onduidelijk, hoofdzakelijk door beperkingen van de experimentele methodologie. Extra kennis van mechanisch geïnduceerde groei en remodellering stelt ons in staat om de ontwikkeling van het hart alsmede het verloop van een ziekteproces beter te begrijpen en het resultaat van verschillende therapieën op de lange termijn te voorspellen.

In dit proefschrift beschrijven wij de ontwikkeling en toepassing van wiskundige modellen om extra inzicht te verkrijgen in de relatie tussen lokale mechanische belasting van het weefsel, lokale weefselgroei en -remodellering alsmede veranderingen in de globale pompcapaciteit van het hart. Omdat de mechanische belasting van het hartspierweefsel voor een belangrijk deel bepaald wordt door het oriëntatiepatroon en de massa van de spiervezels in de wand hebben wij ons bij het modelleren met name gericht op spiervezelgroei en -heroriëntatie.

Directe bepalingen van de hartspiervezeloriëntaties zijn op dit moment alleen ex vivo mogelijk. Het patroon van spiervezeloriëntaties heeft echter een grote invloed op de vervorming van de wand. Met name de longitudinale gradiënt in rotatie (rotatiegradiënt in apex-basis richting; torsie) is sterk afhankelijk van het transmurale verloop in spiervezeloriëntaties. Als gevolg van deze relatie tussen de vervorming van de wand en de spiervezeloriëntaties in de wand, kan het meten van de vervorming van de wand ons indirect meer informatie over de spiervezeloriëntaties geven. In hoofdstuk 2 presenteren wij een experimentele studie waarin met behulp van 'magnetic resonance tagging' (MRT) noninvasief en in vivo de lokale torsie van het linker ventrikel (LV) op verschillende longitudinale niveaus is bepaald. In een normale controlegroep was de torsie gedurende de ejectiefase van de hartcyclus voor alle longitudinale niveaus ongeveer gelijk en had een negatief teken. Dit duidt erop dat in het normale LV het transmurale verloop in spiervezeloriëntatie ongeveer gelijk is voor alle niveaus. Tevens hebben wij lokale LV torsie bepaald in een groep met situs inversus totalis (SIT). Bij deze aandoening zijn zowel de positie als de globale anatomie van de organen linksrechts gespiegeld. De functie van het hart is voor zover wij weten normaal. In tegenstelling tot in de normale controlegroep, vonden wij in de groep met SIT dat LV torsie, gemiddeld gezien, langs de longitudinale as van teken verandert; bij de 
basis had de torsie een positief teken, terwijl bij de apex torsie ongeveer gelijk was aan die in het normale LV. Deze resultaten impliceren dat het transmurale verloop in spiervezeloriëntaties in het geval van SIT bij de basis gespiegeld is, net als de globale anatomie, maar dat dit patroon bij de apex hetzelfde is als in het normale hart.

In hoofdstuk 3 beschrijven wij een studie waarin wij stimulus-respons relaties voor LV spiervezelgroei en -heroriëntatie onderzocht hebben. In een relatief simpel tweedimensionaal (2D) model werden deze relaties onderzocht op hun mogelijkheid het holte- en wandvolume, het patroon van spiervezeloriëntaties en de torsie van het LV te reproduceren. Gebaseerd op experimentele bevindingen zoals beschreven in de literatuur hebben wij aangenomen 1) dat in het geval van overstrekking van de spiervezels aan het begin van de ejectiefase van de hartcyclus of in het geval van een verhoogde spiervezelcontractiliteit de wandmassa toeneemt om de rek en de contractiliteit van de spiervezels te verlagen, 2) dat wanneer er grote spiervezelrekken zijn het holtevolume toeneemt om de rek weer te laten afnemen, en 3) dat spiervezels heroriënteren om hun rek gedurende de ejectiefase van de hartcyclus te normaliseren en om hun verschil in oriëntatie met de aanliggende spiervezels te minimaliseren. Vervolgens hebben wij met het model spiervezelgroei en -heroriëntatie gesimuleerd in zowel het normale als het SIT LV. Hierbij werd gestart met een onrealistisch holtevolume en wandvolume en een onrealistisch patroon van spiervezeloriëntaties. In het normale LV ontstond er na spiervezelgroei en -heroriëntatie een stabiele situatie met een realistisch patroon van spiervezeloriëntaties, een realistische LV torsie en realistische LV holteen wandvolumes. Ook in de simulatie van het SIT LV ontstond er een stabiele situatie waarin de oriëntaties van de spiervezels kwalitatief goed overeenkwamen met de (beperkte) histologische data. Net als in de experimenten van hoofdstuk 2 veranderde LV torsie in het SIT model van teken in longitudinale richting. Ook de voorspelde waarden van holte- en wandvolume waren realistisch en kwamen overeen met waarden gevonden in de simulatie van het normale LV. Het was dus mogelijk op basis van mechanisch geïnduceerde groei en remodellering van de spiervezels de experimentele observaties van spiervezeloriëntaties, torsie en holte- en wandvolumes in zowel het normale LV als in het SIT LV te reproduceren.

In hoofdstuk 4 hebben wij een complexer driedimensionaal (3D) eindige-elementenmodel gebruikt om een gedetailleerde hypothese over heroriëntatie van de spiervezel te onderzoeken. De hypothese was dat de oriëntatie van de spiervezel lokaal zo wordt aangepast dat de afschuiving van de spiervezel ten opzichte van zijn aangrenzende spiervezels minimaal is. In een testsimulatie zijn wij gestart met een structuur waarin, in overeenstemming met het $2 \mathrm{D}$ model, spiervezels geen transmurale component in hun oriëntatie hadden (initiële spiervezelrichtingen liepen parallel aan het endo- en epicardium). Na heroriëntatie was er een oriëntatiepatroon ontstaan met transmurale componenten in oriëntatie die binnen de spreiding van experimentele data, zoals bepaald met 'magnetic reso- 
nance diffusion tensor imaging' (MRDTI), lagen. Als gevolg van deze transmurale componenten in oriëntatie namen op lokaal niveau de gemiddelde spiervezelrek en maximum spiervezelspanning gedurende de ejectiefase toe, terwijl verschillen in spiervezelrek en -spanning afnamen. Op globaal niveau was het LV, ondanks een gelijkblijvende wandmassa, in staat om een hogere druk te ontwikkelen en meer volume te ejecteren. Deze simulatie laat zien dat afschuiving van de spiervezels mogelijk een belangrijke rol speelt tijdens de ontwikkeling van het patroon van spiervezeloriëntaties en het tegelijkertijd homogeniseren van spiervezelspanning en -rek over de wand. Tevens laat deze simulatie zien dat de (spatiële distributie van de) transmurale component in de spiervezeloriëntatie een belangrijke factor is voor zowel de lokale mechanische belasting van het weefsel als voor de globale pompcapaciteit van het LV.

Bij de vondst dat de voorspelde spiervezelrichtingen binnen de experimentele spreiding liggen moet worden aangetekend dat door onnauwkeurigheid van MRDTI de spreiding in de meetresultaten relatief groot is $\left( \pm 6^{\circ}\right)$. Vanwege de hoge gevoeligheid van lokale longitudinale en transmurale gradiënten in rotatie voor de spiervezelrichtingen is een vergelijking tussen model en experiment wat betreft deze rotatiegradiënten een meer kritische test. Daarom hebben wij de longitudinale en transmurale gradiënten in rotatie zoals verkregen met het model voor en na spiervezelheroriëntatie vergeleken met waarden verkregen uit MRT experimenten (hoofdstuk 5). Wij vonden dat na heroriëntatie van de spiervezels de gesimuleerde longitudinale en transmural gradiënten in rotatie aanzienlijk dichter bij de experimentele waarden lagen. Onze hypothese van spiervezelheroriëntatie leek dus niet alleen de spiervezeloriëntaties, maar ook de longitudinale en transmurale gradiënten in rotatie te kunnen voorspellen. Echter, er bleven verschillen bestaan tussen model en experiment.

Met het simpeler 2D model (hoofdstuk 3) hadden wij gevonden dat gecombineerde spiervezelgroei en -heroriëntatie voldoende was om een stabiele LV structuur en geometrie te krijgen. In het 3D eindige-elementenmodel van hoofdstukken $\mathbf{4}$ en $\mathbf{5}$ was groei niet meegenomen. Daarom was de volgende stap een methode te ontwikkelen/onderzoeken om inhomogene groei met het 3D eindigeelementenmodel te simuleren. In dit soort modellen is een spanningsvrije configuratie nodig ter referentie van de vervorming en de daaruit resulterende spanningen. Het is gebruikelijk om bij het modelleren van groei deze initiële configuratie als vaste referentie te gebruiken gedurende het gehele groeiproces. Wij redeneerden dat afbraak en opbouw van het weefsel deze referentie in de tijd zal veranderen en dat daarom het gebruik van een veranderende referentie mogelijk realistischer is. In hoofdstuk $\mathbf{6}$ beschrijven wij een studie waarin wij het effect onderzochten van een in de tijd veranderende of een in de tijd vaste referentie op de groei van het LV. Met zowel een veranderende als een vaste referentie waren wij in staat om ten minste $30 \%$ volumegroei te simuleren. Met een vaste referentie nam de stimulus (afwijking van eind-diastolische spiervezelrek ten opzichte 
van een homeostatische waarde) toe, resulterend in een heterogenere verdeling van eind-diastolische spiervezelrek. Echter, bij een veranderende referentie nam de stimulus af en werden de verschillen in eind-diastolische spiervezelrek over de wand kleiner. Omdat in werkelijkheid een relatief homogene spiervezelrek wordt gemeten, lijkt het realistischer om groei te simuleren met gebruik van een veranderende referentie. Met geen van de twee manieren kon echter een stabiele eindsituatie bereikt worden met compleet homogene spiervezelrek.

Concluderend, wij hebben wiskundige modellen ontwikkeld en gebruikt om groei en remodellering van het hartspierweefsel te onderzoeken. In een 2D model vonden wij dat mechanische stimulering van spiervezelgroei en -heroriëntatie voldoende was om een realistische geometrie en structuur van het LV te verkrijgen. Dit resultaat suggereert een primaire rol voor mechanische belasting in de ontwikkeling en onderhoud van de structuur van de hartspier. Onze 3D simulaties gaven aan dat afschuiving van de spiervezels een van de belangrijkste mechanische stimuli voor spiervezelheroriëntatie in het LV kan zijn. Tevens lieten de simulaties zien dat de transmurale component van de spiervezeloriëntatie een significante invloed heeft op de pompcapaciteit van het hart.

In ons 3D eindige-elementenmodel was het niet mogelijk om de experimenteel bepaalde longitudinale en transmurale gradiënten in rotatie volledig te reproduceren. In overeenstemming met wat wij vonden in het 2D model, zal dit waarschijnlijk komen omdat in het 3D model alleen spiervezelheroriëntatie als adaptief proces werd gebruikt, en andere processen zoals groei afwezig waren. Om die reden hebben we een methode ontwikkeld om inhomogene driedimensionale groei te simuleren. In de toekomst kan uitbreiding van het 3D model met zowel groei- als meerdere remodelleringsmechanismen helpen om een beter (kwantitatief) inzicht te verkrijgen in de relatie tussen lokale mechanische belasting, lokale groei en remodellering van het weefsel, alsmede verandering in pompcapaciteit van het hart. 

Dankwoord 
Zie zo, de Kroon op het werk is klaar. Er rest mij alleen nog de mensen te bedanken zonder wiens inzet en begeleiding het onderzoek en dit bijbehorende proefschrift niet mogelijk waren geweest.

Allereerst gaat mijn dank uit naar het promotieteam: Prof. Theo Arts, Dr. Tammo Delhaas en Dr. Peter Bovendeerd.

Beste Theo, sinds jouw eerste college hartmodelleren was ik definitief gegrepen door de hartmechanica. Ik ben blij dat ik de kans heb gekregen om met jou mijn promotieonderzoek te doen. Tijdens mijn onderzoek heb je mij met onze talloze, altijd interessante discussies enorm geholpen. Je legde de lat altijd hoog, terwijl de drempel om iets te vragen bij jou altijd laag was. Die combinatie heb ik als zeer prettig ervaren. Ik had mij geen betere mentor kunnen wensen. Bedankt ook voor de retraiteweken. Bedankt dat je mijn promotor wilde zijn!

Beste Tammo, de eerste keer dat wij kennis maakten, merkte ik al dat je geen blad voor de mond neemt. Die directe manier van communiceren, en het enthousiasme dat je uitstraalt over onderzoek (met name over situs inversus totalis, SIT) werkte aanstekelijk. Ik ben blij dat wij uiteindelijk een paar mooie papers over SIT in het American Journal of Physiology hebben kunnen publiceren. Bedankt voor jouw bijna onuitputtelijke inspanningen om mijn teksten te verbeteren. Naast jouw begeleiding bij de tot stand koming van de wetenschappelijke output, waardeer ik enorm de vriendschappelijke sfeer van het 'serieus ouwehoeren' die jij mede op het lab creërde. Die sfeer zal ik gaan missen. Tammo bedankt.

Beste Peter, van het promotieteam ken ik jou het langst. Jij was de eerste die mijn interesse wekte voor de hartmechanica. Tijdens mijn promotieonderzoek was je voor mij de stevige Eindhovense rots in de Maastrichtse branding. Met name bij de eindige elementen studies was je voor mij onmisbaar. Jouw didactische inbreng tijdens onze gesprekken heeft mij veel duidelijkheid gegeven. Wat geldt voor Tammo en Theo, geldt ook voor jou. Naast het doelgerichte overleg was er bij jou altijd ruimte voor het ... minder doelgerichte. Die persoonlijke omgang heeft mij erg goed gedaan. Bedankt daarvoor.

Leden van de beoordelingscommissie, ik begin met voorzitter Prof. Frans van de Vosse. Frans, bedankt dat je de voorzittersrol op je wilde nemen. Tevens bedankt dat je het eerste jaar van deze promotie bij de afdeling biomedische technologie van het academisch ziekenhuis Maastricht (BMT-azM) mogelijk hebt gemaakt. Beste Prof. Ger van der Vusse, Prof. Adriana Gittenberger-de Groot en Prof. Harry Crijns, ik ben mij bewust dat dit proefschrift waarschijnlijk een anders, meer technisch, proefschrift is dan u normaal onder ogen krijgt. Daarom extra bedankt voor uw inspanningen om dit proefschrift kritisch door te lezen en te beoordelen. Prof. Omens, thank you for judging my thesis and for participating in my public defence. 
Joost, de afgelopen jaren was je voor mij als mede-hartmodelleur op het lab de ideale 'sparringpartner'. Zonder jouw, in de praktijk gebrachte, gedachtespinsels (soms elke 15 minuten) zouden de afgelopen jaren een stuk moeizamer voorbij gegaan zijn. Kon ik ook meteen mijn gedachten op tafel leggen. Dat hielp het serieuze onderzoek aardig te relativeren. Voor mij was het logisch om jou als paranimf aan mijn zij te hebben. Super dat je het wilde doen! Paul, ik ben blij dat wij, ondanks het feit dat wij sinds ons afstuderen minder vaak contact hebben, nog steeds goede vrienden zijn. Ook jij bedankt dat je mijn paranimf wilde zijn.

Gustav en Jos, ik wil jullie graag bedanken voor jullie inspanning bij de metingen van de spiervezelrichtingen. Helaas heb ik deze niet in mijn proefschrift kunnen verwerken. Ik hoop dat er in de toekomst nog een mooie publicatie in zit.

Borut, thank you for visiting Maastricht. Our conversations were enlightening. Koen, Nico, Jos en Irene, bedankt voor de vele gesprekken op het lab met een voor mij ideale verhouding tussen serieuze- en wat minder serieuze onderwerpen.

(Oud-)collega's van Biofysica, Fysiologie en BMT-azM groep die ik nog met naam genoemd heb: bedankt voor de leerzame en gezellige tijd. Collega's van de TUe, met name de kamergenoten van WH 4.11, ook jullie bedankt.

Verder wil ik mijn vrienden van harte bedanken. Ik weet dat voor sommige (zo niet voor de meeste) van jullie dit onderzoek een ver-van-me-bed-show is, maar zonder jullie vriendschap buiten de wetenschap was dit proefschrift een stuk saaier tot stand gekomen.

Tenslotte wil ik mijn broer en mijn ouders bedanken. Bruurke, bedankt voor je steun de afgelopen jaren. Pa en ma, bedankt voor het onvoorwaardelijke vertrouwen dat jullie mij altijd gegeven hebben. Zonder de steun, de kansen en de mogelijkheden die jullie mij geboden hebben was dit proefschrift nooit geschreven.

Wilco Kroon, februari 2009 

Curriculum Vitae 


\section{JACOB WILLEM KROON}

Born June 71981 in Velp, The Netherlands.

\section{Education}

1993-1999 Atheneum, Thorbecke college, Arnhem, The Netherlands

1999-2004 Biomedical Engineering, Eindhoven University of Technology

Nov-dec. '02 Internship: Development of a method to construct a threedimensional finite element mesh of the cardiac ventricles to simulate cardiac muscle contraction

Peter Bovendeerd, PhD, Roy Kerckhoffs, MSc

Apr-jun. '03 Externship: Development of an experimental (mock-loop) setup of the cardiovascular system Karen May-Newman, PhD (San Diego State University), Prof. Bas de Mol, MD, PhD, Peter Bovendeerd, PhD

Aug. '03-'04 Master's research at Vitatron BV, The Netherlands: Development of a computer model to assess the role of cardiac deformation in the genesis of the electrocardiogram Prof. Adriaan van Oosterom, PhD (Catholic University Nijmegen), Peter van Dam, MSc. (Vitatron BV), Prof. Frans van de Vosse, PhD, Peter Bovendeerd, PhD

\section{Experience}

2004-2008 PhD student, thesis title "Mechanocontrol of cardiac growth and remodeling: Mathematical and experimental analysis of normal and situs inversus totalis hearts"

Nov. '04-'05 Department of Biomedical Technology (BMT-azM), University Hospital Maastricht group of Prof. Frans van de Vosse, PhD

Nov. '05-'08 Departments of Physiology and Biophysics, Cardiovasculair Research Institute Maasticht, Maastricht University group of Prof. Theo Arts, PhD

2008-... Post-doctoral researcher, departments of Biomedical Technology and Surgery, Maastricht University Medical Center 


List of publications 


\section{JOURNAL PUBLICATIONS}

Delhaas T, Kroon W, Bovendeerd P, Arts T (2008) Left ventricular apical torsion and architecture are not inverted in situs inversus totalis. Prog Biophys Mol Biol 97:513-519.

Delhaas T, Kroon W, Decaluwe W, Rubbens M, Bovendeerd P, Arts T (2008) Structure and torsion of the normal and situs inversus totalis cardiac left ventricle. I. Experimental data in humans. Am J Physiol Heart Circ Physiol 295:H197H201.

Kroon W, Delhaas T, Bovendeerd P, Arts T (2008) Structure and torsion of the normal and situs inversus totalis cardiac left ventricle. II. Modeling adaptation to mechanical load. Am J Physiol Heart Circ Physiol 295:H202-H210.

Kroon W, Delhaas T, Bovendeerd P, Arts T (2008) Computational analysis of the myocardial structure: Adaptation of cardiac myofiber orientations through deformation in three dimensions. Med Imag Anal (in press).

Kroon W, Delhaas T, Arts T, Bovendeerd P (2008) Computational modeling of volumetric soft tissue growth: application to the cardiac left ventricle. Biomechan Model Mechanobiol (in press).

\section{REFEREED PROCEEDINGS}

Kroon W, Delhaas T, Arts T, Bovendeerd P (2007) Constitutive modeling of cardiac tissue growth. In: Sachse FB, Seemann G (Eds) Functional imaging and modeling of the heart 2007, Springer Verlag Berlin/Heidelberg, pp. 340-349 (ISBN13: 978-3-540-72906-8).

Kroon W, Delhaas T, Bovendeerd P, Arts T (2009) Adaptive reorientation of cardiac myofibers: comparison of left ventricular shear in model and experiment. In: Functional imaging and modeling of the heart 2009 (in press).

\section{AWARDS}

ASME Summer bioengineering conference 2008, $3^{\text {rd }}$ place PhD student poster competition: Solid mechanics, design and rehabilitation category. Poster title: Computational analysis of myocardial structure; Shear-induced 3D reorientation of cardiac myofibers. 